\title{
Neural Synchronization in Seizures
}

\author{
A Dissertation \\ Presented to \\ the faculty of the School of Engineering and Applied Science \\ University of Virginia
}

\author{
In partial fulfillment \\ of the requirements for the Degree \\ of Doctor of Philosophy in Chemical Engineering
}

Sarah E. Johnson

May 2012 


\section{APPROVAL SHEET}

The dissertation is submitted in partial fulfillment of the requirements for the degree of

Doctor of Philosophy in Chemical Engineering

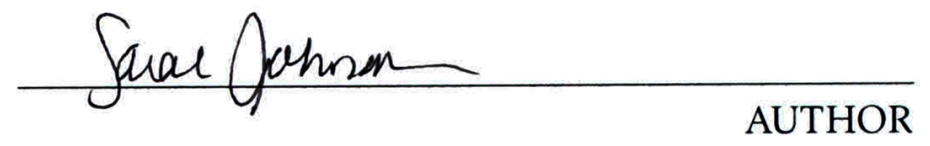

This dissertation has been read and approved by the examining Committee:
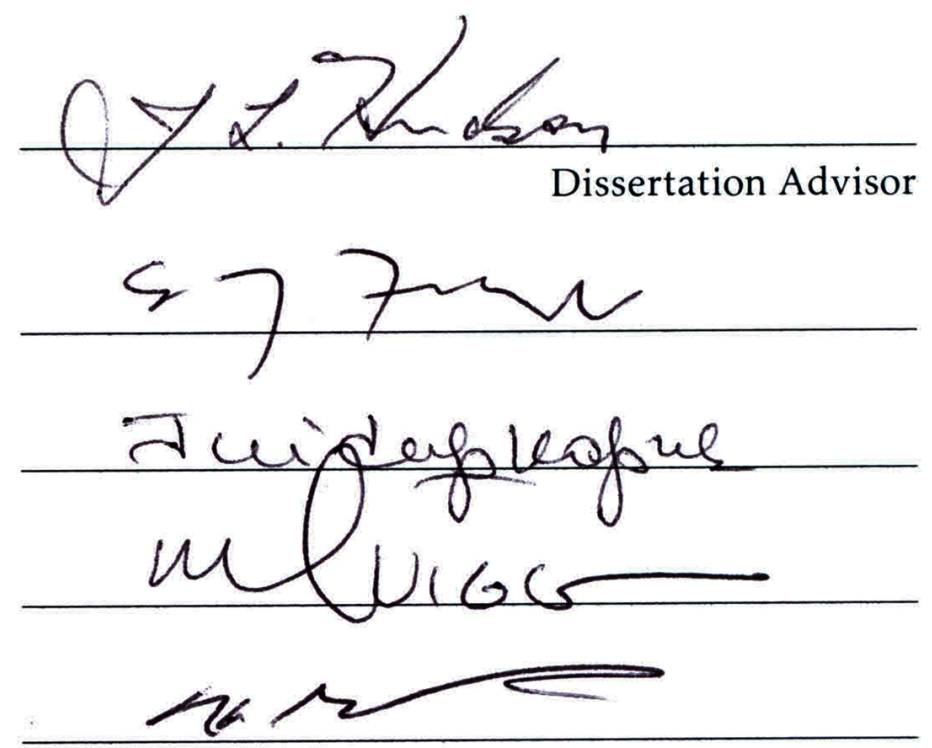

Accepted for the School of Engineering and Applied Science:

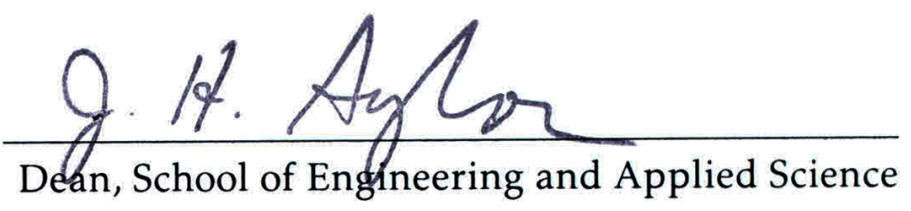

May 2012 


\section{Abstract}

Persons with epilepsy suffer from recurring seizures thought to be the result of abnormal, hypersynchronous neural activity. Understanding the role that synchronization of neural activity plays in the development and propagation of seizures can lead to improvement of current treatments and aid in designing future treatments. This dissertation examines the role of synchronization in the whole brain during seizures, between neurons with feedback, and in pairs of synaptically connected neurons in an in vitro epilepsy model.

The synchronization index (SI), which combines phase synchronization between two electrodes with the synchronization of neurons at an electrode, was calculated for seizures of four patients with medically intractable mesial temporal lobe epilepsy. After recording multiple seizures per patient, patients underwent surgical resection to remove the part of the temporal lobe visually determined to be the focus of their seizures. SI values calculated from the recorded EEGs were mapped onto a diagram of the EEG electrodes. Examination of seizures across all the patients showed the presence of five distinct stages. However, average values of the synchronization measure were higher for patients whose seizures were not completely cured after surgical resection. SI may provide a metric to predict surgical outcome prior to surgery.

Phase-based models were experimentally constructed from a single synaptically 
isolated cultured hippocampal neuron. These models were used to determine the stimulation parameters necessary to produce the desired synchronization behavior in the action potentials of a pair of neurons coupled through a global time-delayed interaction. Measurements made using a dynamic clamp system confirm the generation of the synchronized states predicted by the experimentally constructed phase model. This model was then utilized to extrapolate the feedback stimulation parameters necessary to disrupt the action potential synchronization of a large population of globally interacting neurons. These feedback parameters can be used as a starting point for studies using an animal model.

The event synchronization was calculated for pairs of neurons exposed to decreased extracellular $\mathrm{Mg}^{2+}$. As the concentration of $\mathrm{Mg}^{2+}$ was decreased, the synaptic strength between the cells and within the network was increased as demonstrated by two methods of quantal analysis. However, the level of synchronization had a possible dose response relationship as $\mathrm{Mg}^{2+}$ was decreased. This suggests that increased synaptic strength, while initially increasing the synchronization between pairs of neurons, can only increase synchronization to a point. To further examine the effect of synaptic strength on synchronization three common antiepileptic drugs (AEDs) were added to the epileptic solution. Of the three AEDs, two have mechanisms that alter the synaptic strength. None of the AEDs altered the synchronization significantly. 


\section{Acknowledgments}

I would like to thank my advisor, Professor Hudson, and my minor mentor, Professor Kapur, for their time, patience and guidance over the course of this dissertation. I would also like to thank Drs. Mark Quigg and Gabe Martz for their interest in the Synchronization Index and their efforts in applying this measure to human patients.

Thank you to all the current and past members of the Hudson and Kapur lab groups for the research based and non-research based discussions and for assistance in my research. In particular, I would like to thank Craig Rusin for his role in building and designing the experimental apparatus and initial experiments. I would like to thank Doris Chen, Kendra Keith, Terry Zhang and Suchitra Joshi for providing the cell cultures used in my experiments. Thank you to Xuan Liu who was a great help in the final stages of the SI work. And to Matthew Clark, István Kiss, Karthik Rajasekaran, Suchitra Joshi, Matt Rannals, Carolina Ramôa, Corinne Balint, Karen Blaha and Xin Ren, thank you for taking the time to answer my questions, helping me with the rig, and making the days go faster.

Last, but not least, I would like to thank all of my family and friends. Theresa Bankston, Maura Fierro, Masha Azmovia, Michael van den Bossche and Rose CoxGalhotra for being great listeners, giving me encouragement and brightening my days. My parents, sisters, aunts, uncles, cousins, grandparents and in-laws, thank you for being such wonderful support for me and my family while we were so far 
away from home. I'm so grateful for your visits and phone calls. And my wonderful husband and children who make everyday special and fun; I could not have done this without your support.

This work was funded through NIH, the Biotechnology Training Program and the Biomedical Innovation Fund/Ivy Foundation at UVA. 


\section{Contents}

1 Introduction 1

2 Background 3

2.1 Neurophysiology ....................... 3

2.2 Seizures and Epilepy $\ldots \ldots \ldots \ldots$. . . . . . . . . . . . 7

2.3 Synchronization and Phase . . . . . . . . . . . . . 11

2.4 Scope and Outline of this Dissertation . . . . . . . . . . 12

3 Synchronization of EEG Signals $\quad 13$

3.1 Introduction . . . . . . . . . . . . . 13

3.2 Background ........................... 14

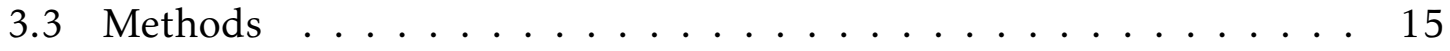

3.3.1 Synchronization Index . . . . . . . . . . 15

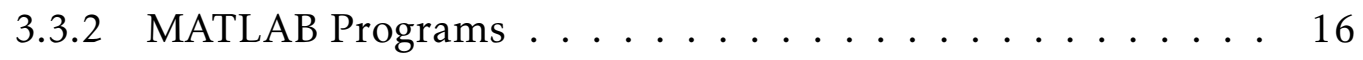

3.3.3 Subjects and Seizures ................... 18

3.3.4 Intracranial EEG Recording . . . . . . . . . . . . . . 19

3.3 .5 Data Analysis ..................... 19

3.3 .6 Statistical analysis ..................... 22

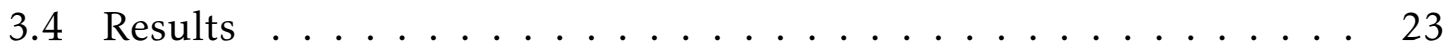

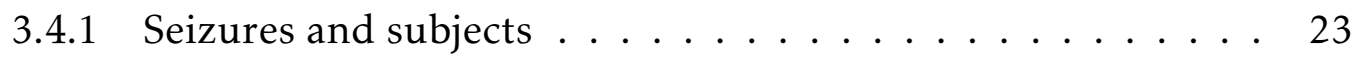


3.4.2 SI connectivity animations and network stages . . . . . 23

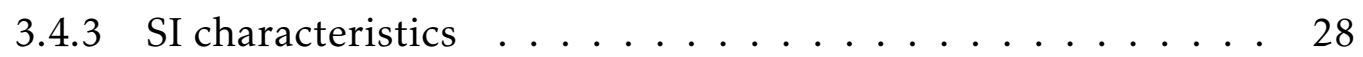

3.4.4 Clinical Correlations ................ 33

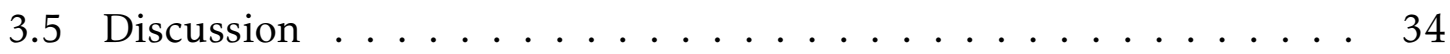

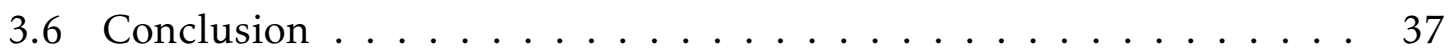

4 Synchronization Using Global Feedback 38

4.1 Introduction $\ldots \ldots \ldots \ldots \ldots \ldots$

4.2 Background .......................... 40

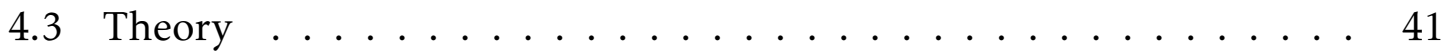

4.4 Methods .......................... 44

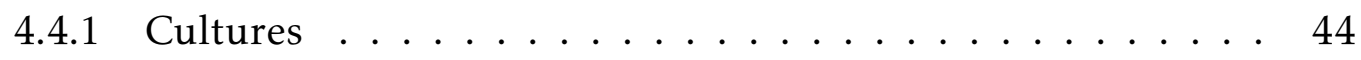

4.4.2 Electrophysiology ....................... 45

4.4.3 Experimental Apparatus . . . . . . . . . . . . 47

4.5 Results ........................... 48

4.5.1 Measurement of the Interaction Function . . . . . . . . . 48

4.5.2 Synchronization of Two Neurons under Global Feedback . . 52

4.5.3 Predictions for a Large Population of Neurons . . . . . . . . . 54

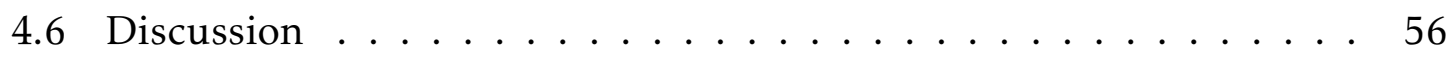

4.7 Conclusions ........................... 59

5 Synchronization of Bursting Activity $\quad 60$

5.1 Introduction $\ldots \ldots \ldots \ldots$

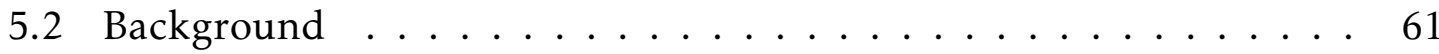

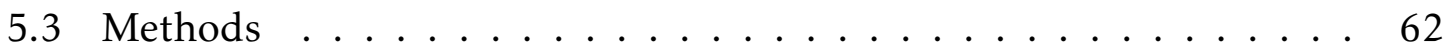

5.3.1 Mixed Culture Preparation . . . . . . . . . . . 62

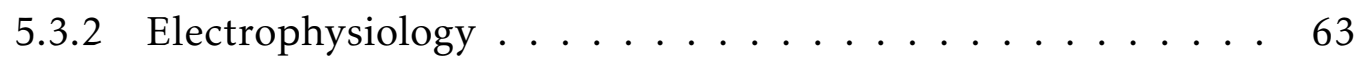




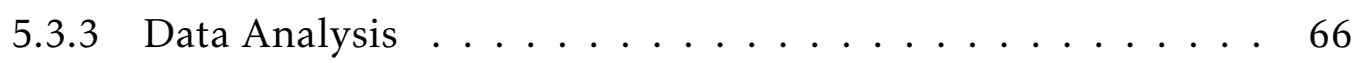

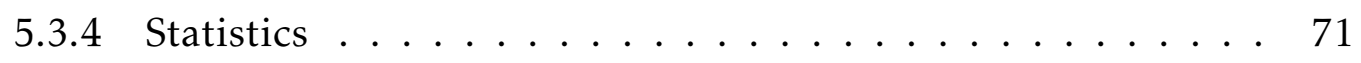

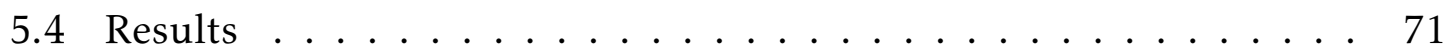

5.4.1 Neuronal Properties ................ 71

5.4.2 Event Synchronization ................ 76

5.4.3 Effect of Decreased Magnesium on Synchronization _ . . . . 81

5.4.4 Effect of Decreased Magnesium on Synaptic Strength . . . 85

5.4.5 Effect of Antiepileptic Drugs on Synchronization . . . . . . 88

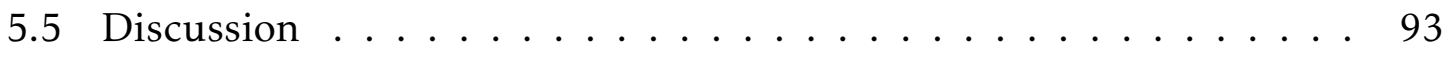

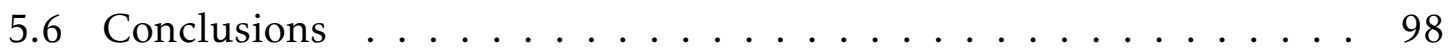

6 Conclusions and Future Work 99

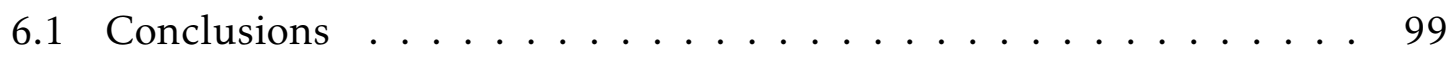

6.2 Future Work ........................ 100

6.2.1 EEG Analysis Based on SI Calculations . . . . . . . . . . 100

6.2.2 Extension of Phase Model and Feedback Design . . . . . . . 101

6.2.3 Synaptic Strength and Synchronization . . . . . . . . . 103

$\begin{array}{ll}\text { A MATLAB Code } & 106\end{array}$

A.1 Calculate SI from EDF files . . . . . . . . . . . 106

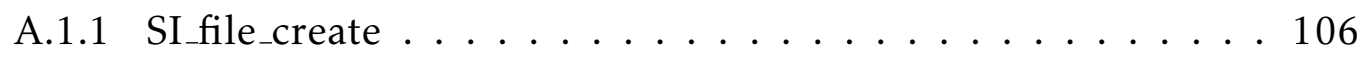

A.1.2 cheby2_filter_func ................. 109

A.1.3 partsplot5 ....................... 110

A.1.4 getsigma ............................ 111

A.1.5 analyze_quick ..................... 111

A.2 Create SI Movies and Still Images . . . . . . . . . . . . . 112

A.2.1 make_image ....................... 112 
A.2.2 make movie . . . . . . . . . . . . . 121

A.2.3 still_image $2 \ldots \ldots \ldots \ldots$. . . . . . . . . . . 124

A.2.4 Examples for the Different Image Modes . . . . . . . . . . 126

References 


\section{List of Figures}

2.1 A neuron is made up of a soma (cell body), dendrites and axon. . . . 3

2.2 Schematic of an electrical synapse and a chemical synapse. . . . . 4

2.3 Schematic showing the location of the temporal lobe and hippocam-

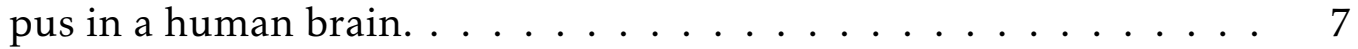

2.4 Schematic demonstrating the pathway of spacial memory. The circuit from the entorhinal cortex through the hippocampus is also a pathway used by seizures. . . . . . . . . . . . . .

2.5 Schematic of seizure activity in the brain. The surface EEG signal is compared to activity measured extracellularly and intracellularly

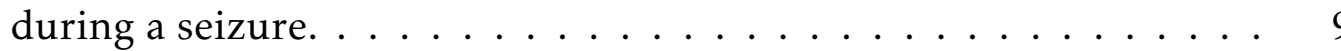

2.6 Schematic of seizure spread for a complex partial seizure and a partial seizure secondarily generalized. . . . . . . . . . . . . . 10

3.1 Electrode placement and schematic representation for SI animations. 20

3.2 Maximal SI values per electrode sufficiently represent the distribution of all SI values. Overlapping plots demonstrate that the distribution of maximal SI values over time mimics that of the global SI distribution. A representative seizure from each patient is displayed. 25

3.3 Seizure network stages manifested by maximal SI connections. . . . 26 
3.4 Relative Durations of Network Stages During Seizures. For each subject and for all subjects together (Group), average relative durations of each SI network stage are displayed as percentage of seizure time.

3.5 Normalized average SI values for each seizure across network stages. For all seizures from each subject, the normalized average maximum SI value across all electrodes is displayed for each network stage. . . 30

3.6 Hippocampal and Temporal Cortical Electrodes SI value distributions during Seizures. Electrodes with highest average nMaxSI correlated well with area of seizure manifestation by visual analysis. . . . . . . 31

3.7 Average SI value at Seizure Hub is lower among subjects with postsurgical seizure freedom. . . . . . . . . . . . . . . . 34

4.1 Schematic of the separated dynamic clamp system. . . . . . . . . 46

4.2 The interaction function is measured by applying self-delayed feedback to a single patch clamped neuron. . . . . . . . . . . 50

4.3 Experimentally measured interaction function and response functions for three different neurons predict and in-phase and anti-phase synchronization states for two coupled neurons. . . . . . . . . . 51

4.4 Experimental results for two patch clamped neurons under linear global feedback. . . . . . . . . . . . . . . . 53

4.5 Eigenvalues calculated for balanced cluster states as a function of feedback stimulation delay. . . . . . . . . . . . . . . . 57

5.1 Schematic of the dual patch clamp apparatus. . . . . . . . . 65

5.2 Examples of firing behaviors for different cells in CM. . . . . . . 73

5.3 Bursts in LM and ZM were identified using the $\log _{10}$ ISI histogram. . 75 
5.4 Example of the detection of an excitatory response and a failure for a single action potential . . . . . . . . . . . 77

5.5 Examples of connection types seen in the dual current clamp experi-

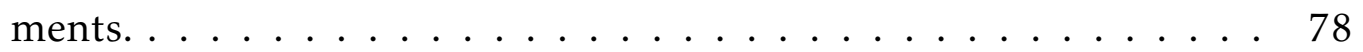

5.6 Dual whole cell recordings in current-clamp mode. . . . . . . . . 79

5.7 Dual whole cell recordings in current-clamp mode for surrogate data. 81

5.8 Dual whole cell recordings in current-clamp mode for CM and ZM conditions. Grouped experiments showed no significant difference between synchronization of action potentials in CM and ZM conditions. 82

5.9 Dual whole cell recordings in current-clamp mode for the same pair of cells in CM and ZM and a different pair in CM and LM. Synchronization was significantly increased from CM to ZM and CM to LM. . . . . . . . . . . . . . . . . . .

5.10 Connectivity was decreased from CM to LM and increased from LM to $\mathrm{ZM}$ in a neuron pair with a strong connection under CM conditions. 87

5.11 Connectivity was increased from CM to LM and from LM to ZM in a pair with a weak connection under CM conditions. . . . . . . . 89

5.12 Dual whole cell recordings in current-clamp mode, cumulative IEI distributions and synchronization for PHT experiments. . . . . . . . 91

5.13 Dual whole cell recordings in current-clamp mode, cumulative IEI distribution and synchronization for GYKI experiments. . . . . . . . 92

5.14 Dual whole cell recordings in current-clamp mode, cumulative IEI distribution and synchronization for LEV experiments. . . . . . . . 94

A.1 Examples of the three different display options for the make image

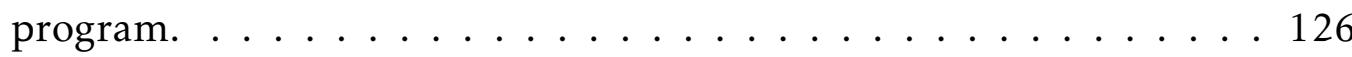




\section{List of Tables}

2.1 Ion concentrations inside and outside the cell and corresponding reversal potentials based on the Nernst Equation at room temperature

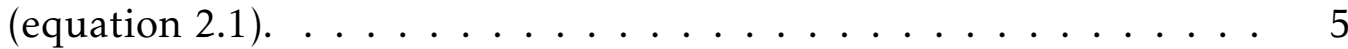

3.1 Clinical Characteristics of Temporal Lobe Epilepsy Subjects . . . . . 24

3.2 Electrodes with higher SI value by stage and subject. Rows indicate all electrodes that displayed significantly elevated nMaxSI during any stage for any subject. . . . . . . . . . . . 32

4.1 Analytical expressions for the real parts of the eigenvalues of the first 4 balanced cluster states, assuming the highest order harmonic in the interaction function is $\leq 6 \ldots \ldots \ldots \ldots$

5.1 Type of connections for grouped and solution-switched experiments 76

5.2 Synchronization and Distances for Dual Current-Clamp Experiments 95 


\title{
List of Symbols
}

\author{
F $\quad$ Faraday constant \\ $I(t) \quad$ Injected current stimulation signal \\ $I_{a p p} \quad$ Baseline injected current \\ $K \quad$ Linear feedback gain \\ M Number of bins used to calculate Shannon entropy \\ m Mean quantal content \\ $n \quad$ Number of action potentials \\ $n_{c} \quad$ Number of individual cells \\ $n_{p} \quad$ Number of cell pairs \\ $p_{i} \quad$ Probability of a phase difference being in the $i$ th bin \\ $Q \quad$ Strength of event synchronization, $0 \leq Q \leq 1$ \\ $q \quad$ Bias of event synchronization, $-1 \leq q \leq 1$ \\ $R \quad$ Gas constant \\ S Shannon entropy
}


$S_{\max } \quad$ Maximum Shannon entropy, $=\ln M$

T Temperature

$t \quad$ Time

$V \quad$ Measured neuron membrane potential

$V_{m} \quad$ Mean value of membrane potential

$z \quad$ Valance

$\alpha \quad$ Gain setting on the patch clamp amplifiers

$\sigma \quad$ Entropy Index

$\tau \quad$ Feedback delay; Event synchronization window 


\section{Chapter 1}

\section{Introduction}

Epilepsy is a disease where patients suffer from recurring, unprovoked seizures and it is estimated that epilepsy will affect around 3\% of the United States population who live to be 80 years old [1]. Traditionally seizures are thought to be the result of abnormal, hypersynchronous neural activity $[2,3]$. Treatment for epilepsy typically involves the use of one or more antiepileptic drugs (AEDs) [3].

Approximately one-third of epilepsy patients have medically intractable epilepsy and two-thirds of these patients are helped by surgical resection [4]. The surgeon removes the part of the brain identified as the focus, or initiation point of the seizure. The focus is identified by visual inspection of EEG signals. Doctors looking at the same EEGs could have differing opinions on the location of the focus. Furthermore, some patients are not seizure free after removal of the identified focus, which means that either the focus was not correctly identified or the initiation of the seizure involved a larger network than the area that was removed. Current research is examining the development of networks via synchronization and correlation of activity to understand the onset and propagation of seizures [5-7].

Recently deep brain stimulation of the thalamus and hippocampus has been 
examined for treatment of epilepsy [8-10]. Electrodes are surgically implanted into an area of the brain so that electronic stimulation can be applied with the goal of decreasing or eliminating the effects of the disease [11]. Current stimulation protocols, called 'open-loop' stimulators, can use relatively high amplitude (4-5 V), square wave pulses at frequencies around $130-150 \mathrm{~Hz}[4,10,11]$. This range of frequencies is based on stimulation frequencies used to treat movement disorders [4]. Adjustments to the stimulation parameters are done postoperatively and remain fixed until the next adjustment [12]. Since seizures account for less than $1 \%$ of the sufferer's lifetime [4], 'closed-loop' stimulators are being examined. Closed-loop devices stimulate in response to a seizure and/or can apply feedback based on the current activity [13]. Design of a feedback signal depends on the underlying neuronal activity and the desired activity. Since seizures are thought to be hypersynchronous activity, the feedback signal would be designed to desynchronize a synchronous population of neurons $[14,15]$.

This dissertation looks at the synchronization behavior of neural activity for the treatment of epilepsy. Networks defined by synchronization between intercranial EEG electrodes are compared for four patients with medically intractable epilepsy. Similarities are examined to define key characteristic network maps, while the differences suggest possible predictors of surgical outcomes. Cultured neurons are used to develop a model of neuronal oscillations. A feedback signal is developed that predicts a state of desynchronization for a large population of neurons. Finally, the change in action potential synchronization is examined and compared for an in vitro epilepsy model with the addition of three conventional AEDs. 


\section{Chapter 2}

\section{Background}

\subsection{Neurophysiology}

Neurons are the basic unit of the brain. A neuron has a soma (cell body), dendrites and axons which carry, process and transfer information (signals) from other cells (see Figure 2.1). The information from the presynaptic cell travels down

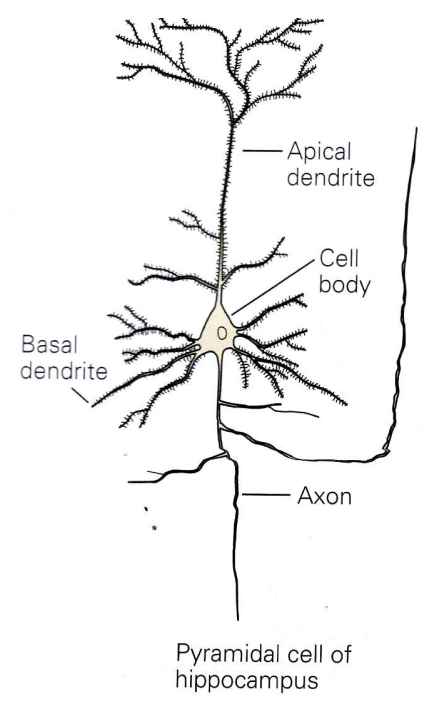

Figure 2.1: A neuron is made up of a soma (cell body), dendrites and axon. Figure from Kandel et al. [1]. 

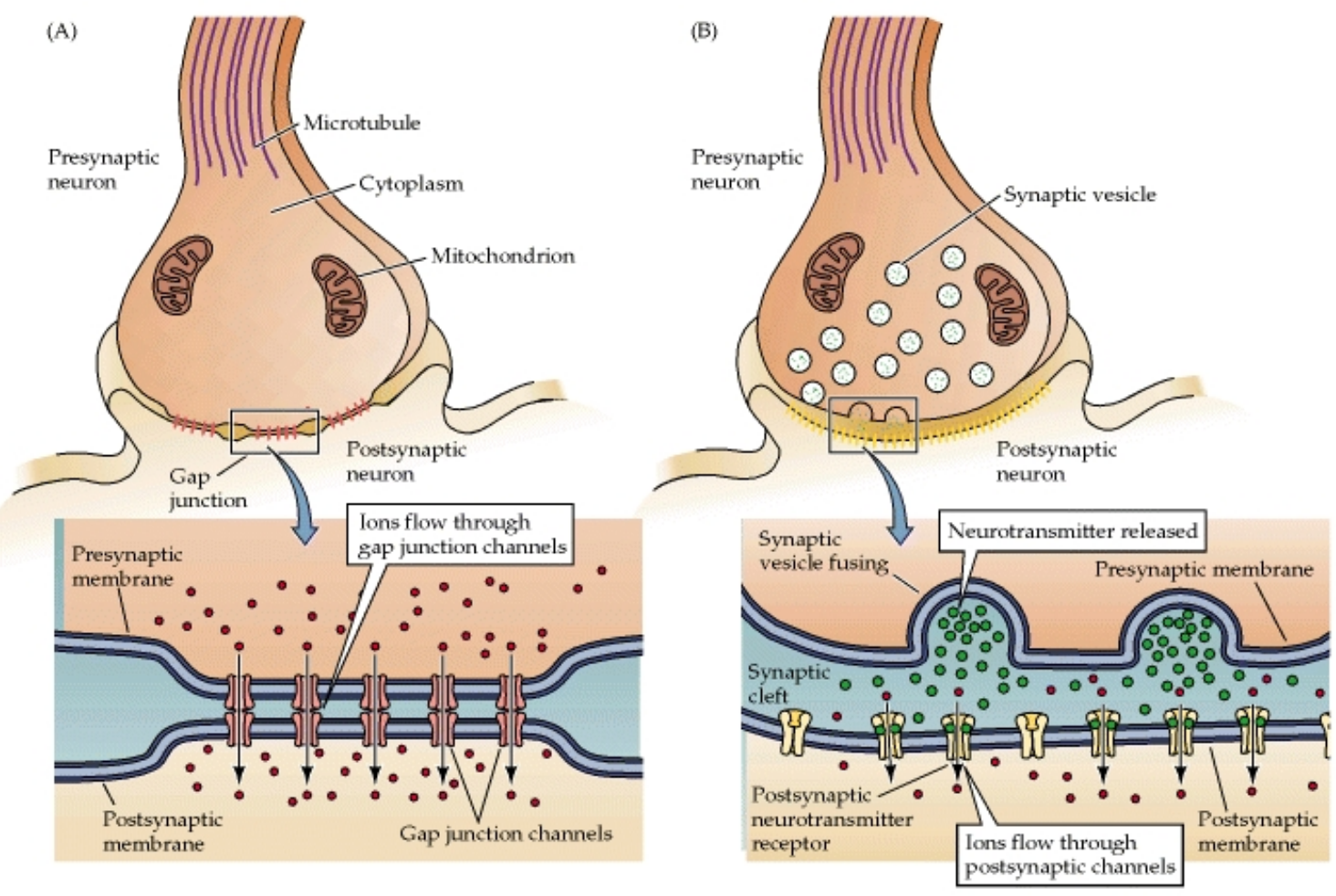

Figure 2.2: Schematic of an electrical synapse (A) and a chemical synapse (B). In an electrical synapse, the membranes of the two neurons are directly connected by gap junctions. In a chemical synapse, the presynaptic neuron releases neurotransmitters which travel across the synaptic cleft and bind to receptors on the postsynpatic neuron $[1,16]$. Figure from Marshall et al. [17].

the dendrites to the soma. In the soma the information is gathered and an action potential may be fired if the potential is large enough. The action potential then travels through the axon to synapses with other neurons. Information is transferred between neurons through the synapses. Synapses can be electrical or chemical. In an electrical synapse, the connection is made through a gap junction (Figure 2.2 A) $[1,16]$. In a chemical synapse, an action potential causes vesicles containing neurotransmitters to fuse to the membrane and release causing a change in membrane potential (Figure 2.2 B) $[1,16]$.

A chemical gradient exists between the inside of the cell and the extracellular 
solution. The reversal (equilibrium) potential $\left(E_{X}\right)$ for each ion can be calculated using the Nernst equation:

$$
E_{X}=\frac{R T}{z F} \ln \frac{[X]_{o}}{[X]_{i}}
$$

where $R$ is the gas constant, $T$ is the temperature, $z$ is the valance of the ion $X, F$ is the Faraday constant, $[X]$ is the concentration of the ion inside $(i)$ and outside $(o)$ the cell. Table 2.1 shows the concentrations for all ions and approximate reversal potentials for $\mathrm{Na}^{+}, \mathrm{K}^{+}$and $\mathrm{Cl}^{-}$for the experiments presented in this dissertation.

With depolarization of the cell, sodium gates open, causing a large depolarization towards the reversal potential of sodium [18]. Sodium channels transition from activated (open), to inactivated (closed—not able to open), to resting (able to open) [1]. The large depolarization caused by the flow of sodium ions into the cell through the open sodium channels is then countered by an increase in potassium conductance. Potassium ions flow out of the cell, which brings the membrane potential back towards rest and the reversal potential of potassium [18]. The inactivation state of sodium channels is longer at depolarized voltages [1]. These two events constitute the firing of an action potential. The action potential travels down the axon to presynaptic terminals.

The depolarization of the presynaptic terminal creates an influx of $\mathrm{Ca}^{2+}$ ions by opening voltage-gated $\mathrm{Ca}^{2+}$ channels. Synaptic vesicles filled with neurotransmitter

Table 2.1: Ion concentrations inside and outside the cell and corresponding reversal potentials based on the Nernst Equation at room temperature (equation 2.1).

\begin{tabular}{lccc}
\hline & Inside $(\mathrm{mM})$ & Outside $(\mathrm{mM})$ & Reversal Potential $(\mathrm{mV})$ \\
\hline $\mathrm{Na}^{+}$ & 3 & 148 & 100 \\
$\mathrm{~K}^{+}$ & 164 & 3 & -103 \\
$\mathrm{Cl}^{-}$ & 11 & 156 & -68 \\
\hline
\end{tabular}


molecules are then fused to the membrane facilitated by proteins sensitive to $\mathrm{Ca}^{2+}$ concentration. The neurotransmitter then crosses the synaptic cleft and binds to postsynaptic receptors. The binding causes the opening or closing of the postsynaptic channels and alters the membrane potential of the postsynaptic cell.

An excitatory postsynaptic potential (EPSP) is created when the neurotransmitter released by the presynaptic cell binds to receptors that open ligand-gated $\mathrm{Na}^{+}$ and $\mathrm{K}^{+}$permeable channels [1]. The membrane potential in the postsynaptic cell is depolarized, or becomes closer to the action potential threshold. An inhibitory postsynaptic potential (IPSP) has a neurotransmitter that binds to receptors that open ligand-gated $\mathrm{Cl}^{-}$channels. This causes a hyperpolarizing response, or a response that pushes the membrane potential away from the threshold. The neurotransmitter is then either broken down or can be reused by the presynaptic neuron via a process called reuptake.

A neuron that releases glutamate as its neurotransmitter acts on excitatory receptors, while a neuron that releases $\gamma$-aminobutyric acid (GABA) mainly acts on inhibitory receptors [1]. Three subtypes of directly gated channels exist for excitatory responses: $\alpha$-amino-3-hydroxy-5-methylisoxazole-4-propionic acid (AMPA), kainate and ( $N$-methyl-D-aspartate) NMDA. GABA acts on two receptors, $\mathrm{GABA}_{\mathrm{A}}$ and $\mathrm{GABA}_{\mathrm{B}}[1]$, however only $\mathrm{GABA}_{\mathrm{A}}$ will be discussed this dissertation.

Neurons in the brain are organized into several regions, the most relevant to this dissertation being the diencephalon and the cerebral hemispheres. The diencephalon contains the thalamus and hypothalamus and the cerebral hemispheres contain the cerebral cortex (split into four lobes), the amygdaloid nuclei, the basal ganglia and the hippocampus [1]. Figure 2.3 shows the location of the temporal lobe and the hippocampus. Each of the structures in the brain have specific purposes. There are many pathways between these structures that give rise to memory, lan- 


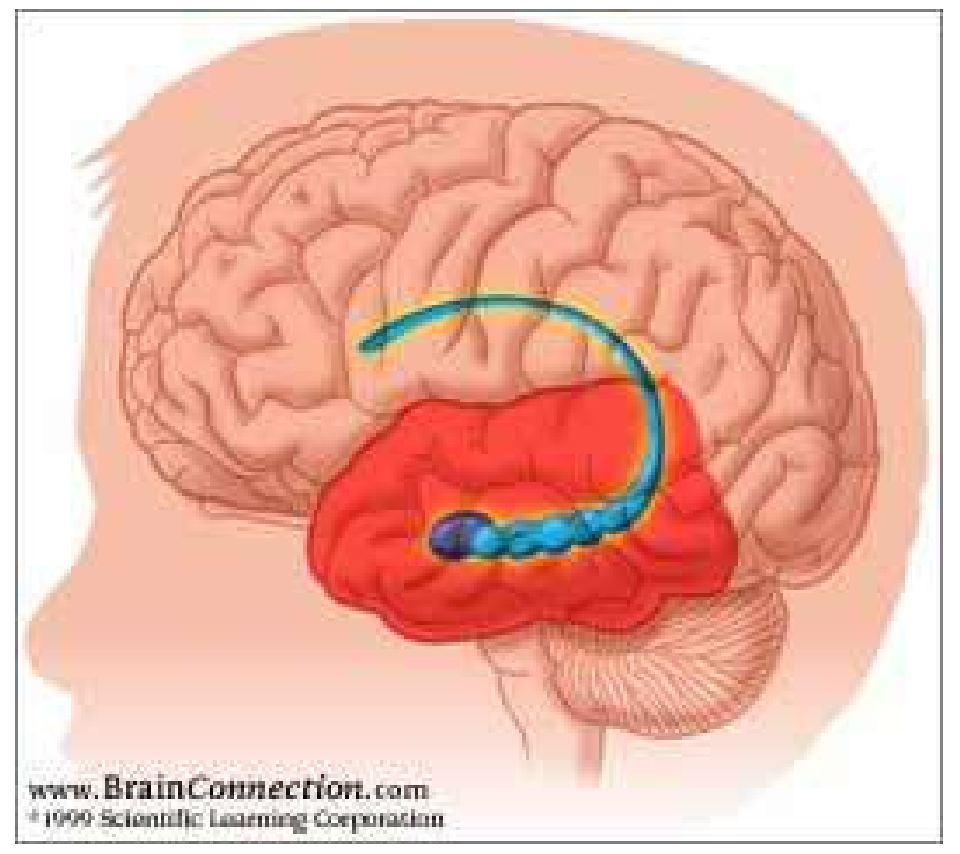

Figure 2.3: Schematic showing the location of the temporal lobe (red) and hippocampus (blue) in a human brain. The purple area is the amygdala. Image from The Brain Bank [22].

guage, the senses, motor skills and cognition. The temporal lobe processes auditory information, and in addition to the hippocampus is in part responsible for memory storage and emotions. Figure 2.4 demonstrates a pathway for spacial memory. Information is received and processed by the respective cortical area before being passed to the entorhinal cortex and then through the hippocampus. These types of pathways can also play a role in the generation and spread of seizures [19-21].

\subsection{Seizures and Epilepy}

A seizure consists of abnormally synchronized activity often in a bursting pattern. The bursting pattern can be seen in single neurons, using intra- or extra-cellular recordings, and in the summed behavior of large groups of neurons, using an electroencephalograph (EEG) recording (See Figure 2.5). The time during the 


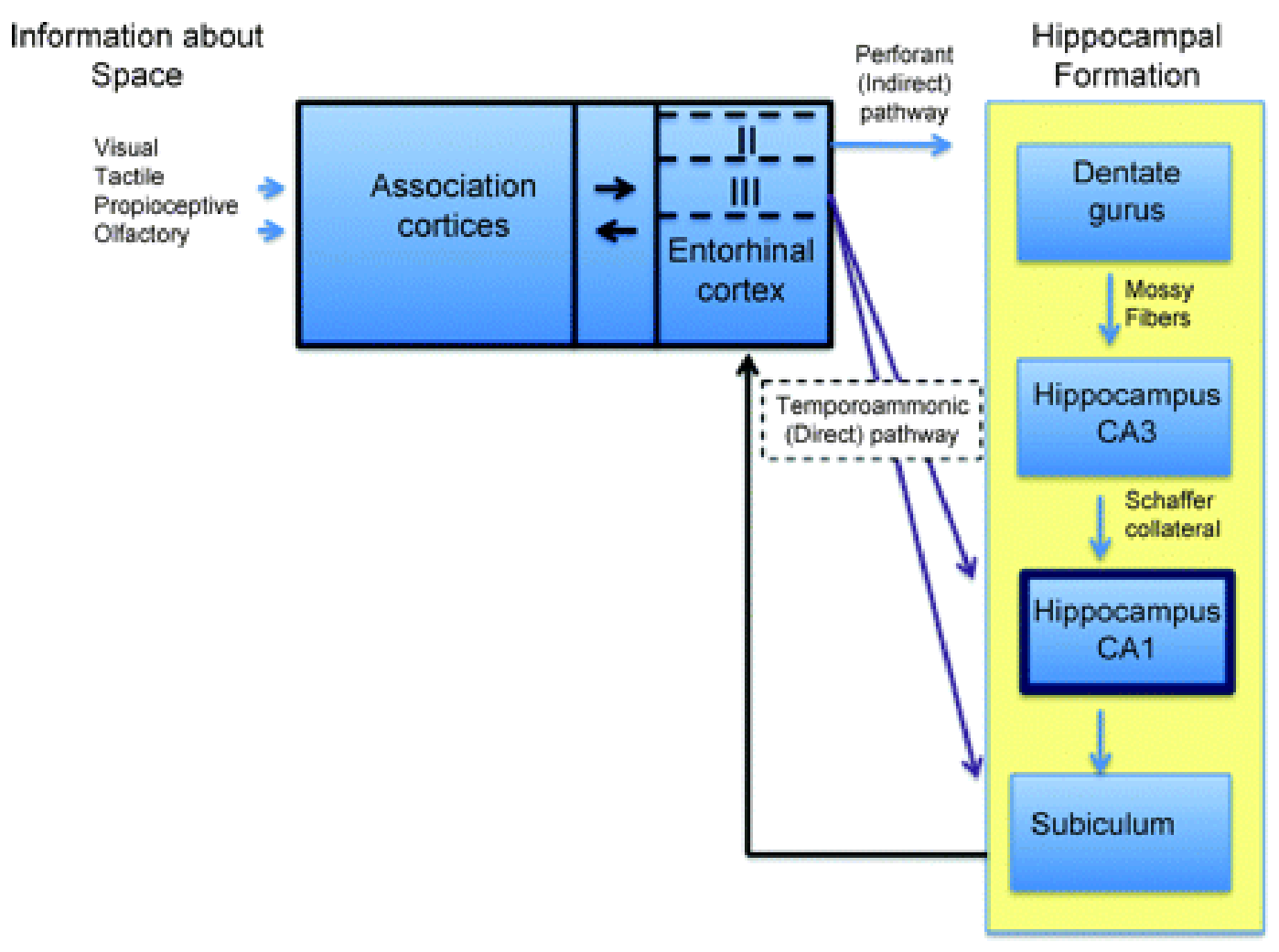

Figure 2.4: Schematic demonstrating the pathway of spacial memory. The circuit from the entorhinal cortex through the hippocampus is also a pathway used by seizures. Figure from Muzzio et al. [23].

seizure is referred to as the "ictal" period. The time immediately before a seizure is "preictal;" immediately after is "postictal;" and the period between seizures is the "interictal" period. The abnormal neural activity can begin in a small area called the focus, and then the synchronized activity will spread to nearby neurons and/or the rest of the brain [2]. Two classes of seizures are discussed in this dissertation, partial seizures and secondarily generalized seizures. Partial seizures start at the focus, but then spread to nearby areas (Figure 2.6 left). If the seizure starts at a focus and then spreads to the whole brain, it is secondarily generalized (Figure 2.6 right). A complex partial seizure impairs consciousness, while a simple partial seizure does not [3].

Epilepsy is a disease where patients suffer from recurrent seizures. Epilepsy 


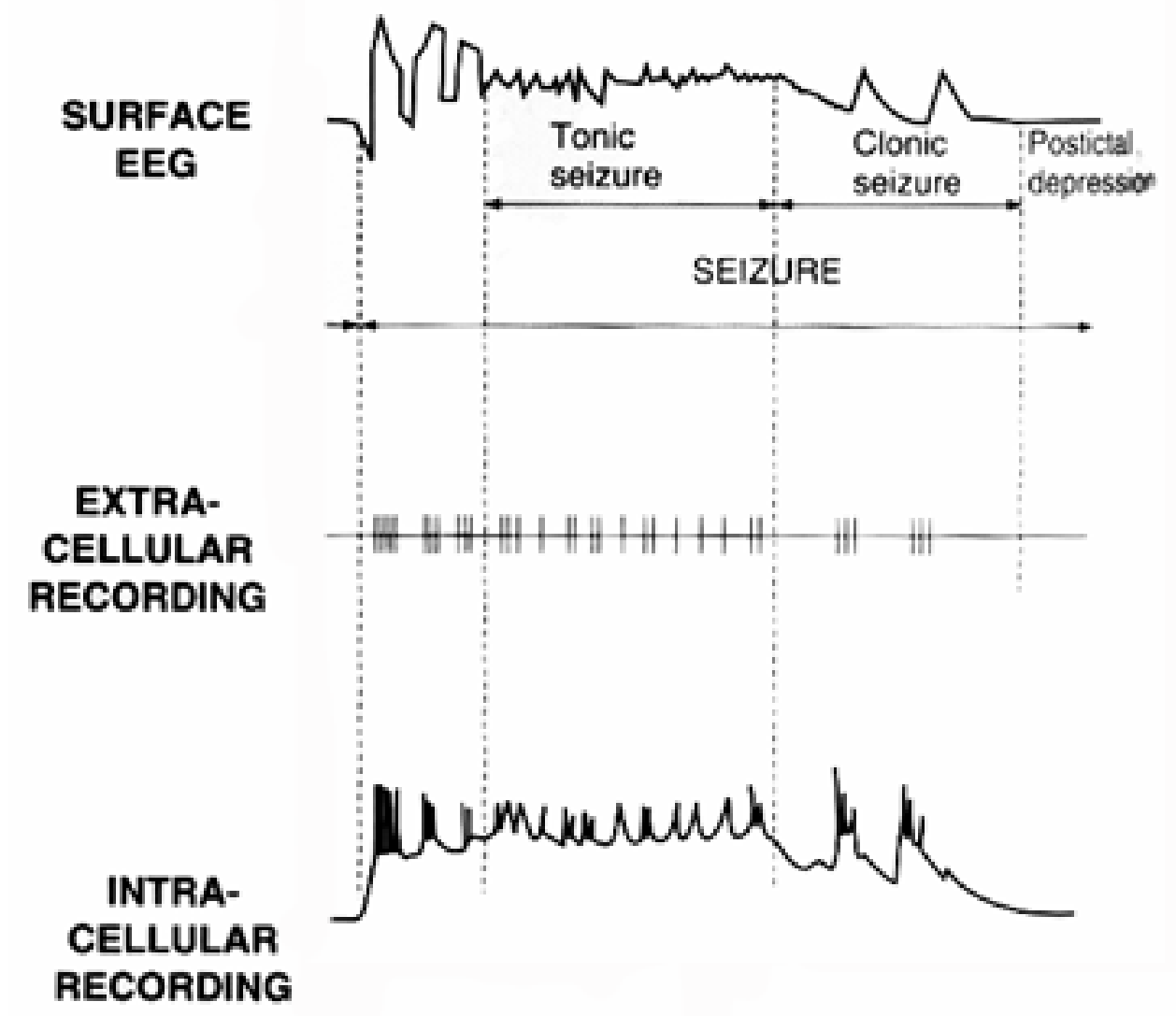

$n^{500 \mathrm{~m} \mathrm{sec}_{-1}}$

Figure 2.5: Schematic of seizure activity in the brain. The surface EEG signal (top) is compared to activity measured extracellularly (middle) and intracellularly (bottom) during a seizure. The EEG is representation of the collective behavior for a large groups of neurons. The extracellular trace shows behavior of a small group of neurons. The intracellular traces shows the activity of one neuron. Adapted from Ayala et al. [24].

syndroms are classified by the type of seizures, age of onset, EEG characteristics, evidence of brain pathology and area of seizure origination [3]. For mesial temporal lobe epilepsy (MTLE), the seizure focus occurs in one or both temporal lobes. These seizures are medically refractory in $20-30 \%$ of patients [3]. If the seizures are not controlled by medicine, surgical resection could be done in which the front part of 


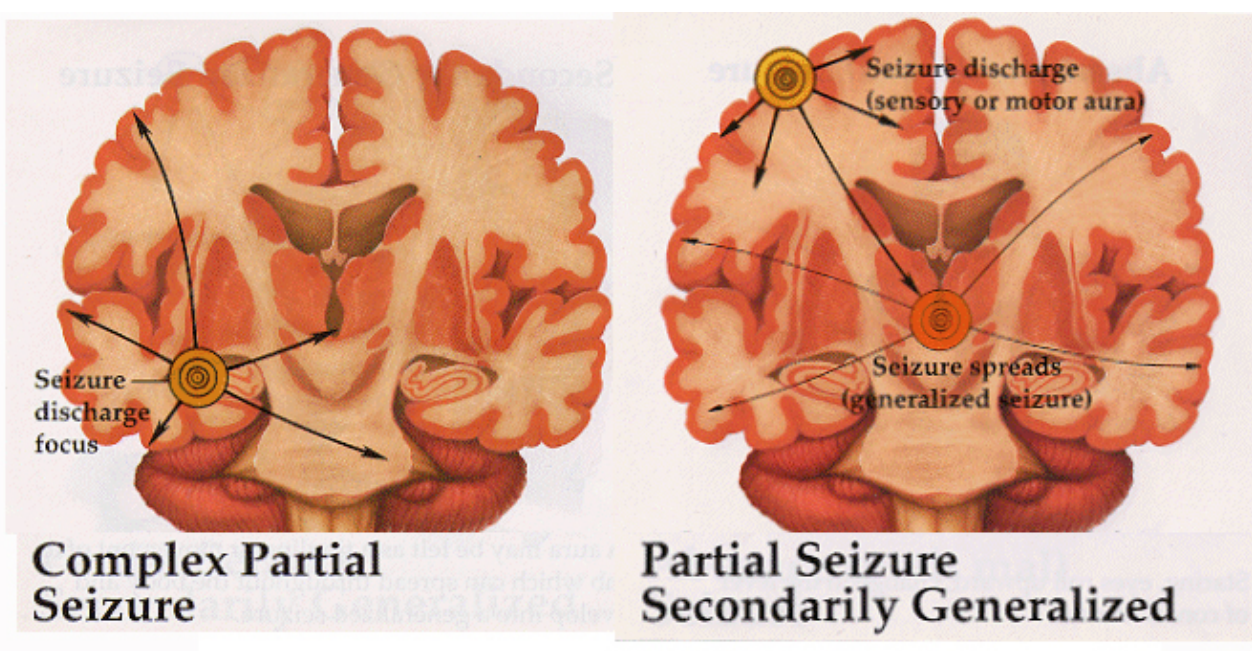

Figure 2.6: Schematic of seizure spread for a complex partial seizure (left) and a partial seizure secondarily generalized (right). Adapted from London Health Science Center [25].

the temporal lobe is removed [1].

Neurostimulation, one alternative to resection, is being studied for possible treatment of epilepsy $[11,12]$. Electrodes are implanted into an area of the brain to provide an electronic stimulation that can decrease or eliminate the seizures [11]. Chronic neurostimulation has recently been shown to reduce seizures in patients with medically intractable epilepsy [10]. Current 'open-loop' stimulation protocols often use relatively high amplitude, square wave pulses $[4,11]$. Furthermore, the amplitude and frequency are set postoperatively and calibrations are done by trial and error. The open-loop stimulations are often continuously applied. Since seizures account for less than $1 \%$ of the sufferer's lifetime [4], this stimulation is most likely being applied more often than is necessary for symptom relief.

In contrast, 'closed-loop' devices can either stimulate in response to a seizure, apply feedback or signals based on the current neuronal activity, or both [13]. Since seizures are thought to be hypersynchronous activity, research has been examining signals that could desynchronize a synchronous population of neurons $[14,15,26]$. 
Individual activity of neurons during seizures has been studied using in vitro epilepsy models and intracellular, extracellular and/or whole cell recording techniques. There are a variety of in vitro epilepsy models and they produce bursting activity with variation in the length and frequency of bursting [27]. Often the synchronization of the bursting activity is examined using cross-correlation [28-30], field potentials [31-33] or by visual inspection [34-38].

\subsection{Synchronization and Phase}

The term 'synchronization' can be used in two slightly different ways. The first way is described by Pikovsky et al. [39] as "adjustment of rhythms of oscillating objects due to their weak interactions." This definition most accurately describes the type of synchronization studied in Chapters $3 \& 4$. In these chapters, the oscillations are periodic and can easily be described using phase. In Chapter 3 the phase is defined by the Hilbert transform (see Equation 3.1). Chapter 4 defines phase linearly from the peak of one action potential to the next. For this definition of synchronization, the change in the phase is of interest, and amplitude is not a necessary factor. The phase difference between the oscillators can take on any value from 0 to $2 \pi$ and they will be considered synchronized as long as the phase difference is relatively consistent.

The second definition is less stringent, where synchronization only implies that defined events are occurring simultaneously or near simultaneously in time. This is the type of synchronization studied in Chapter 5. These neurons often have long quiescent periods, which cannot be described by a phase. The action potentials

are instead defined as discrete events in time and the synchronization is calculated by the near-simultaneous occurrence of the action potentials within a small time 
window.

\subsection{Scope and Outline of this Dissertation}

The goal of this dissertation is to examine synchronization at different levels of the brain to assist in treatment of patients with epilepsy.

Chapter 3 looks at the dynamic behavior described by the SI for four patients with MTLE. Distinct patterns are seen across all seizures and patients. By defining what characteristics are similar in most seizures and patients, future work can focus on the differences in the patterns and relating those differences to clinical outcomes.

Chapter 4 uses feedback in isolated, cultured neurons to demonstrate the ability of a phase model to predict synchronization states of the two neurons when feedback is applied. The two neurons demonstrated in-phase and anti-phase synchronization as predicted by the phase model. For a large group of neurons, parameters for a feedback signal that would create a desynchronized state were determined.

Chapter 5 demonstrates the ability of an in vitro epilepsy model to increase the level of action potential synchronization between two neurons in culture. The application of three conventional AEDs with different mechanisms shows different effects on the synchronization and bursting behavior. Two AEDs attenuate the bursting activity, but one increases the synchronization of action potentials and the other AED shows no effect. The final AED did not stop the bursting activity, but the level of synchronization was slightly decreased. None of the observed changes in synchronization were statistically significant. 


\section{Chapter 3}

\section{Synchronization of EEG Signals During Seizures in Humans ${ }^{1}$}

\subsection{Introduction}

This chapter describes Synchronization Index (SI) as applied to human intercranial EEG measurements during seizures. Patients who have medically intractable epilepsy may have intercranial EEG electrodes implanted to identify the focus (or foci) of their seizures. Surgical resection of the focus may provide relief for these patients. The standard technique of identifying the focus is visual inspection. EEG measurements near the focus tend to show an increase in amplitude early in the start of the seizure. Additionally, more than one electrode may show this increase of amplitude suggesting communication and possible synchronization of the neuron activity in those areas. As a seizure progresses, the large amplitude activity may spread to other electrodes and areas. Preliminary studies using a kindled rat

\footnotetext{
${ }^{1}$ G.U. Martz, S.E. Johnson, X. Liu, B.J. Wolf, J.L. Hudson and M. Quigg, "Display of Ictal Hippocampal-Temporal Cortical Network Dynamics in Refractory Mesial Temporal Lobe Epilepsy." Epilepsy Res., (submitted).
} 
model demonstrated that SI patterns could distinguish between different kindling locations [40]. SI was developed to indicate the increase of amplitude and phase synchronization in the EEG signals for two EEG electrodes. This measurement can assist in identifying the seizure focus and may also demonstrate when surgical resection will or will not benefit the patient.

The overall analysis and patient selection was conducted by Dr. Gabriel U. Martz. SI was developed by István Z. Kiss [15] and here the use of SI is extended to a selected group of patients with mesial temporal lobe epilepsy (MTLE). The main programs described in this chapter can all be found in Appendix A. Xuan Liu assisted with additional programming and making movies for all of the patients. Bethany Wolf provided the statistical analysis.

\subsection{Background}

One hypothesis to account for the third of patients with refractory mesial temporal lobe epilepsy (MTLE) with continued seizures after epilepsy surgery [41-43] is that surgery may merely interrupt an epileptic network rather than removing a discrete "epileptic focus" [44-48]. Understanding emergent network behavior may help identify the structures critical to initiation, propagation, and termination of seizures for individual patients.

A method of examining ictal network topology is analysis of synchronization of EEG patterns among brain regions [49-53]. For example, some studies have revealed high synchrony in structures important to seizure initiation [54-57]. Others suggest that interactions between mesial structures and neocortex are critical for seizure initiation [58-60].

The purpose of this study was to validate a method of quantitative network 
topology in a sample of patients with MTLE with histopathologically-confirmed hippocampal sclerosis and known clinical outcomes. The measure used in this study was the Synchrony Index (SI), a measurement of the strength of oscillator coupling [15]. Preliminary studies showed that SI values increase dramatically during seizures, and tend to be maximal in the area of seizure onset [61]. Examination of SI topology during seizures in this well-defined, homogeneous sample allowed the visualization and quantification of epileptic networks in seizure initiation, propagation and termination.

\subsection{Methods}

\subsubsection{Synchronization Index}

SI is a combination of two measures: synchronization of the neurons comprising the EEG signal, and phase synchronization between two EEG signals. The synchronization of the neurons comprising the EEG signal is defined by the amplitude of the signal. The phase synchronization between two EEG signals is defined by $\sigma$ (see Equation 3.4). The phase, $\phi_{k}$, and amplitude, $r_{k}$, of the $k^{\text {th }}$ EEG signal, $s_{k}$, are calculated as

$$
\begin{aligned}
& \phi_{k}(t)=\arctan \frac{H_{k}(t)}{s_{k}(t)} \quad \text { and } \\
& r_{k}(t)=\sqrt{s_{k}(t)^{2}+H_{k}(t)^{2}}
\end{aligned}
$$

where $H_{k}(t)$ is the Hilbert transform:

$$
H_{k}(t)=\frac{1}{\pi} \int \frac{s_{k}(\tau)}{t-\tau} \mathrm{d} \tau .
$$


The phase synchronization between two oscillating elements can be calculated by the Entropy Index [62],

$$
\sigma=\frac{\left(S_{\max }-S\right)}{S_{\max }}
$$

where $S$ is the Shannon entropy, $S=-\sum_{i} p_{i} \ln p_{i}$ and $S_{\max }=\ln M$, where $M$ is the number of bins and $p_{i}$ is the probability of the phase difference being in the $i$-th bin. A $\sigma$ value of 1 corresponds to complete phase synchronization and a value of 0 is complete desynchronization.

SI is calculated for two EEG signals, $k$ and $l$, as [15]:

$$
S I_{k, l}(t)=\sigma_{k, l} \frac{r_{k}(t)+r_{l}(t)}{2} .
$$

To calculate SI, non-overlapping windows of 1 second were used to calculate $\sigma_{k, l}$ and the average $r_{k}$ and $r_{l}$. A bin number $(M)$ of 30 was used.

\subsubsection{MATLAB Programs}

User defined programs were written in MATLAB to load the EEG data, calculate SI, output basic information for statistical analysis and provide visual representations of the data. The original programs were written by Dr. Kiss, but they have been heavily modified by myself and Xuan Liu.

The first function, SI file_create_newformat_May_2011 (SI_file_create), loads the EEG file data (in .edf form), creates and saves the SI, sigma, amplitudes and electrode names (from the EEG file header). SI_file_create calls partsplot5 which calls get_sigma. These two functions create the amplitudes, sigma and SI values for two electrodes over a time window. SI_file_create also allows the user the option to input a filter range. The program cheby2_filter_func uses a Chebyshev Type II filter, which has a ripple in the stopband and a flat passband, and the program 
also has a $60 \mathrm{~Hz}$ notch filter. The current parameters for the Chebyshev filter are set to an order of 4 and a stopband ripple magnitude of $-20 \mathrm{~dB}$. To make running large numbers of EEG files easy, Run_all_patients_May_2011 allows the user to set a number of input variables to SI_file_create such as filter range and sampling frequency. Additionally, the "good" electrodes for the current 18 patients are automatically set based on the EEG file name.

The next set of functions are used to generate visual displays of SI for all the patients. First, a standardized map of the electrodes used from all of the current patient data was created by Dr. Martz. The location for each electrode was saved as electrode_map.mat and an excel file for each patient was made indicating which electrodes were present during their seizure recordings. For each recorded seizure (EEG file) there is a different start time and length of seizure. An excel file was created to store the time points relative to each file, such as seizure start time, length of seizure, start time for movie (60 seconds before seizure start time) and stop time for the movie (20 seconds after the end of the seizure). Then it calls the make_movie program using the inputs from the excel file to specify start and stop times for the movie. For each movie time period (from start time to stop time) the maximum SI, average SI and minimum SI over all the electrode pairs are recorded. Additionally the visual maximum is set to the 95th percentile of the all of the data being shown in the animation and the minimum is set to $0 \mu \mathrm{V}$.

For every second, an image is plotted with make_image that displays the maximum SI for each electrode and then connects that electrode with its maximum SI pair electrode. The lines and electrodes are colored according to their SI value. Electrodes that are not present in a patient are not plotted. Then make_movie adds the time relative to the file and, in parenthesis, the time relative to the start of the seizure along with the minimum SI, average SI and minimum SI for all the data 
in the movie. The individual frames are then put together to make a movie. For snapshots, the program still_image uses the same inputs as make_movie, with the addition of the time point that an image is wanted. An image is produced similar to the frame in the movie without the time on the frame.

The program make_image can also display the data in two other ways: showing the ' $x$ ' number of maximum SI values or showing the ' $x$ ' number of maximum SI connections with respect to a single electrode. Both ways allow for an adjustable number of electrode pairs to be plotted and neither requires that every electrode has a value associated with it. Unused electrodes that are part of the patients array but have SI values that are not used are plotted in black. Plotting the top ' $x$ ' number of maximum SI electrode pairs may demonstrate that the maximum SI values are concentrated around a certain region or electrode. Plotting the ' $\mathrm{x}$ ' number SI connections for a single electrode can, for example, show how an electrode changes from being synchronized with electrodes near itself to being synchronized with electrodes across the brain. Examples of the different modes can be seen in Figure A.1 in Appendix A.

These programs were made to accommodate possible future directions and research. For the results in this chapter, only 4 patients were fully analyzed as described below. Videos of representative seizures for each patient, plus two additional videos demonstrating the other display options for Subject A's seizure, are available at www. people.virginia.edu/ seb3h.

\subsubsection{Subjects and Seizures}

This retrospective, IRB-approved study evaluated patients with pre-operative diagnosis of medically-intractable MTLE who required intracranial monitoring because of insufficient localization after standardized, noninvasive presurgical evaluation 
including inpatient scalp video-EEG, MRI, neuropsychological battery, and interictal and ictal single-positron emission computerized tomography. All patients had unilateral hippocampal sclerosis on MRI. All had anterior temporal lobectomy and were followed for at least 2 years. Hippocampal sclerosis was confirmed with postoperative histopathology.

\subsubsection{Intracranial EEG Recording}

Patients were implanted with bilateral, 8 contact depth electrodes with $1 \mathrm{~cm}$ spacing (Adtech, Racine, WI) inserted occipitally, extending through the hippocampus and terminating in the entorhinal cortex. Bilateral subdural strips with 4-8 contacts, spaced $1 \mathrm{~cm}$ apart, were placed across anterior, lateral, and posterior frontal lobes and anterior, inferior and lateral temporal lobe regions (Figure 3.1 A). Continuous video-EEG (Grass/Telefactor, Warwick, RI) was recorded at $200 \mathrm{~Hz}$ (60 Hz notch filter, high pass $1 \mathrm{~Hz}$, low pass $70 \mathrm{~Hz}$ ). All seizures were visually reviewed by a board certified neurophysiologist (Dr. Gabe Martz or Dr. Mark Quigg) for determination of the time and location of electrographic seizure onset and offset, clinical seizure onset time and severity [simple partial (SPS), complex partial (CPS) or secondarily generalized (GTC)], and behavioral state (sleep/wake). Processed EEG segments began one minute prior to electrographic seizure onset and ended 20 seconds after seizure termination. Only electrode placements common among all patients were considered for quantitative analysis.

\subsubsection{Data Analysis}

SI was calculated for the entire frequency band $(1-70 \mathrm{~Hz})$ in non-overlapping one second time bins for every possible pair of electrodes in an iterative fashion. 


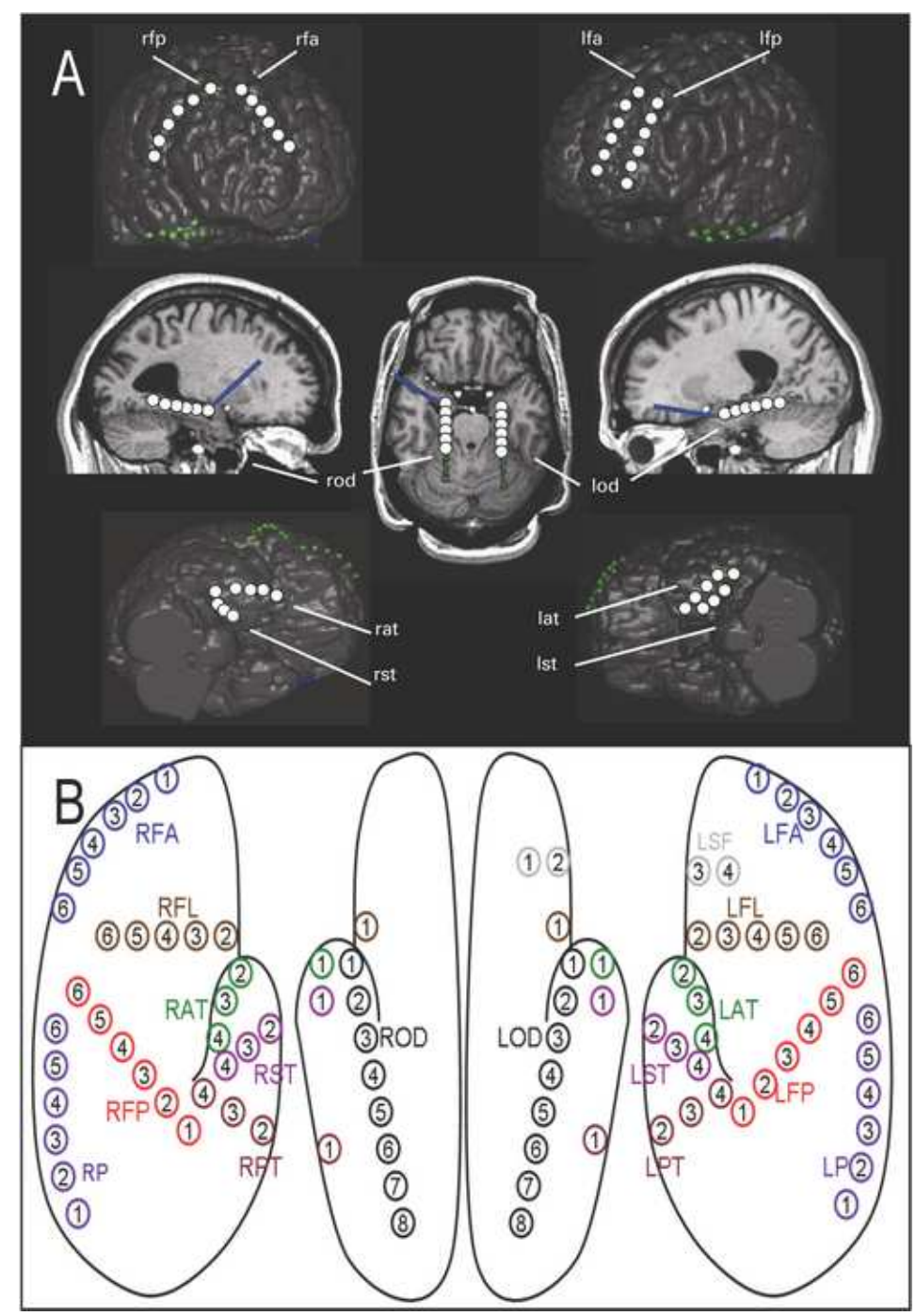

Figure 3.1: Electrode placement and schematic representation for animations. (A) All subjects had bilateral, occipitally-inserted hippocampal depth electrodes. For subdural strip electrodes, exact locations were variable but all patients had at least the bifrontal and temporal cortical strips pictured. (B) For each second of time, Synchrony Index values were mapped onto the corresponding electrodes on this schematic brain template. Not all electrodes were present in all subjects. $\mathrm{R}=$ right, $\mathrm{L}=\mathrm{left}$. Subdural Strip Electrodes: $\mathrm{FA}=$ frontal anterior, $\mathrm{FL}=$ Frontal Lateral, $\mathrm{FP}=$ Frontal Posterior, $\mathrm{P}=$ Parietal, $\mathrm{SF}=$ Subfrontal, $\mathrm{AT}=$ Anterior Temporal, $\mathrm{ST}=$ Subtemporal, PT=Posterior Temporal. Occipitally placed depth electrodes: OD. 
Since the number of interactions among all electrode pairs is large (the number of interactions for a typical 70 electrode acquisition $=$ channels $*($ channels -1$) / 2=$ $\left.70^{*} 69 / 2=2,415\right)$, we determined in preliminary studies that an efficient method of displaying SI connectivity was through "connectivity animations" that allowed direct observation of time-dependent changes across the electrode array. For each electrode at each second, the highest SI value pair was plotted, indicating location and magnitude, upon a brain schematic (Figure 3.1B), thus yielding an animated map of the highest connectivity for each electrode at each second. To demonstrate relative values of SI among electrode pairs, and to highlight statistically significant outlier electrodes, SI amplitude was represented on a normalized 255-bit color scale from low to high ( $\mathrm{red}=$ highest $5 \%$ of SI values). Connectivity animations from every seizure from all patients underwent blinded review.

Because this technique is novel, standard EEG terminology was avoided to distinguish animation from EEG interpretations. "Focality" was defined as 1-2 electrodes that featured visually increased SI amplitude for $\geq 3$ seconds. Changes in SI amplitude occurring in all electrodes simultaneously were considered "global". A "hub" was defined as a spatial array in which $\geq 15$ electrodes were connected to a single, central electrode for $\geq 3$ seconds. This process lead to the description of "stages" of SI connectivity based on empiric patterns of SI amplitude and spatial connectivity that were agreed upon by mutual subsequent review by the research group. Stages were correlated with standard visual analysis of EEG, with time zero set to the seizure onset as determined by standard EEG analysis. Stage durations were analyzed as per cent time $(\% \mathrm{~T})$, calculated by dividing each stages duration by the total duration of the connectivity animation/EEG sample. 


\subsubsection{Statistical analysis}

To enable intersubject and interseizure statistical comparisons, several steps were performed for normalization of the maximum SI values used in connectivity animations.

- Normalized Maximal SI (nMaxSI): for each electrode at all time points, the maximum SI value (regardless of which other electrode was paired with it for that value at that second) was divided by the largest observed maximum SI score of any electrode within that seizure and subject. The resultant nMaxSI ranged from $0-1$.

- Average nMaxSI: the mean value of nMaxSI of a specific electrode confined within an explicitly stated parameter (e.g. seizure, subject, stage or electrode).

- Global average SI: mean nMaxSI across multiple explicitly stated parameters (e.g. average nMaxSI of all electrodes over all seizures for one subject, during a specific stage).

Differences in nMaxSI for each stage and electrode, and for stage by electrode interaction were evaluated using generalized linear mixed models (GLMMs) within and across subjects, treating seizure as a random effect. For statistical comparison of SI distributions of electrodes, SI values for a theoretical average electrode were generated using the mean nMaxSI of all electrodes at each second in time. Contrasts exploring differences between electrodes and stages were conducted via comparisons to the theoretical average electrode value. Associations between nMaxSI and clinical variables (surgical outcome, seizure severity, side of surgery, gender and behavioral state) were similarly explored. The Tukey-Kramer method was used to adjust for multiple comparisons [63]. A GLMM was used to compare stage durations (\%T) 
across subjects. All analyses were conducted using SAS 9.2 (SAS Institute, Cary $\mathrm{NC})$.

\subsection{Results}

\subsubsection{Seizures and subjects}

Four subjects with a total of 25 seizures met criteria Table 3.1. Three subjects had SPS and CPS, while one had CPS and GTC. Clinically determined seizure onset zones (SOZ) were ipsilateral with hippocampal sclerosis on MRI and with subsequent temporal lobectomy. Notably, seizures from subject B consistently had brief right hippocampal onset, followed by predominantly left hippocampal representation on intracranial EEG, and underwent right temporal lobectomy. The other three subjects had more typical unilateral temporal lobe seizures. Two subjects were Engel Class 1a outcome at 2 years (seizure free), while two were Engel Class 1d (seizure free with AED).

\subsubsection{SI connectivity animations and network stages}

The maximum SI per each electrode regardless of its pair were plotted on an electrode map, indicating which electrodes were paired together with the color of the connections and electrodes displaying the magnitude of SI. The maximum SI per electrode is an accurate representation of how the global SI distribution changes over time (Figure 3.2).

Connectivity animations revealed 6 distinct stages, defined by consistent, stage specific, SI amplitude and spatial patterns (see Section 3.3.5). Stages were monotonic and evident in the majority of seizures from all subjects (Figure 3.3). 


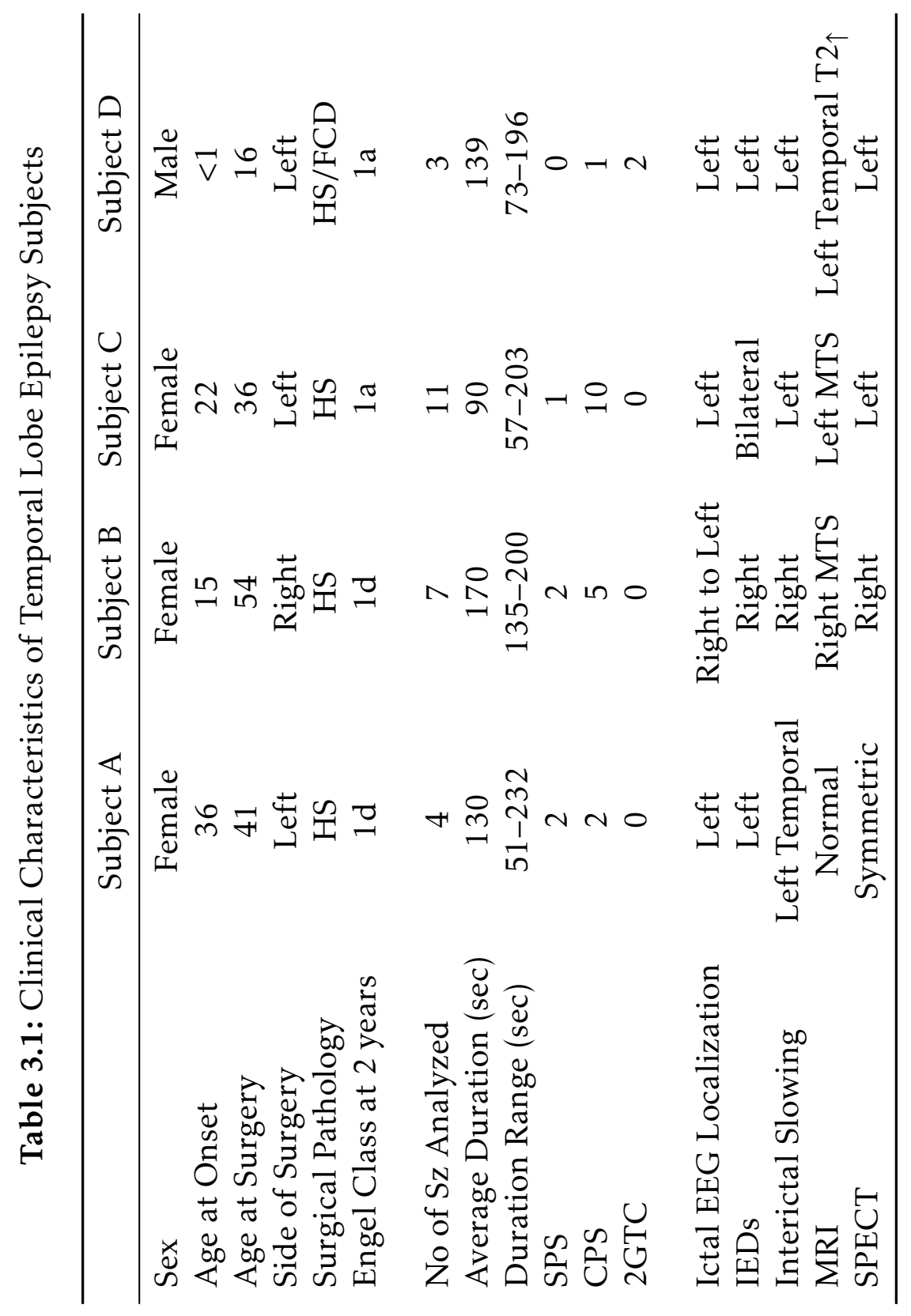




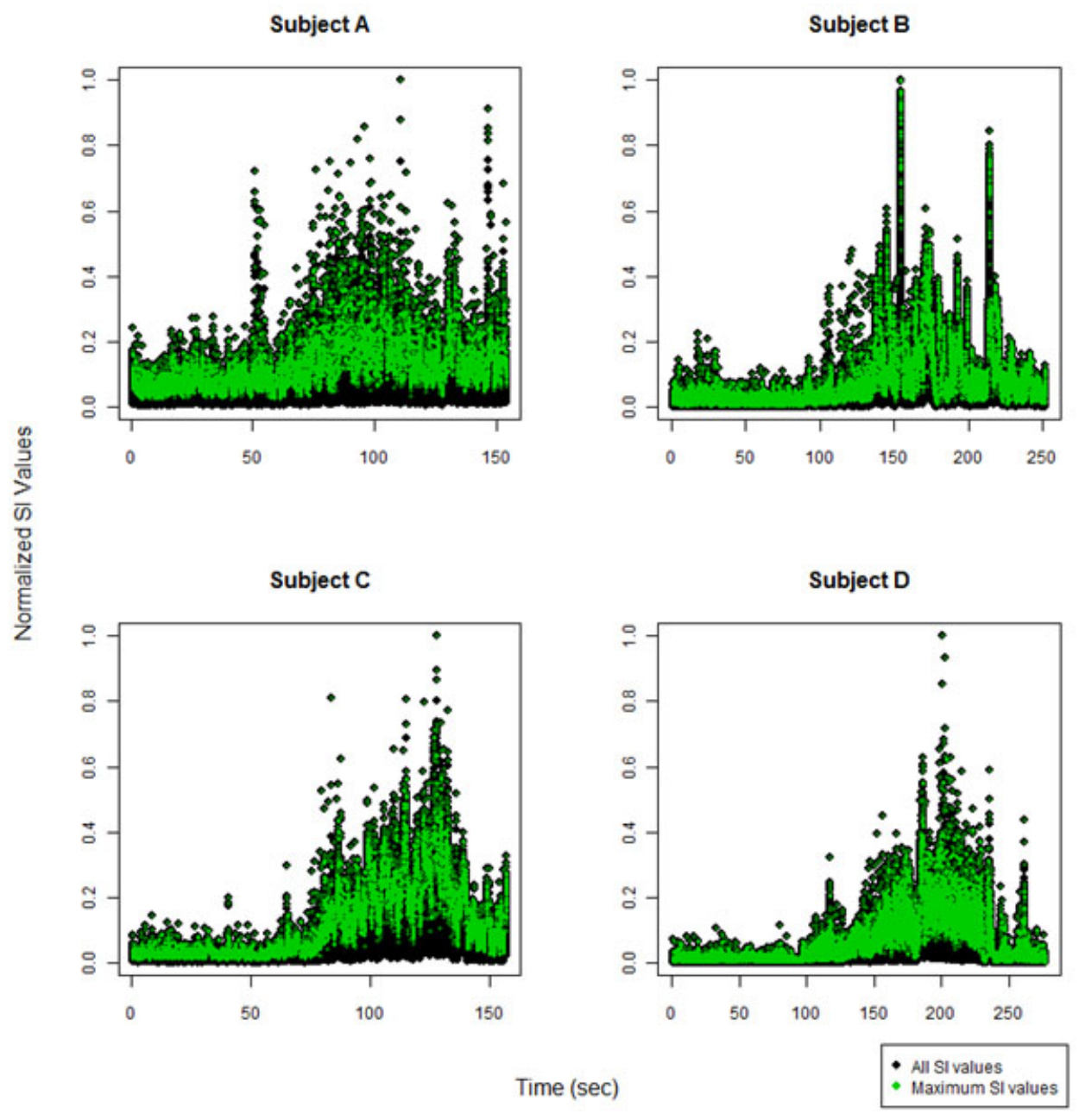

Figure 3.2: Maximal SI values per electrode sufficiently represent the distribution of all SI values. Overlapping plots demonstrate that the distribution of maximal (green dots) SI values (y axis) over time (x axis) mimics that of the global SI distribution (black dots). A representative seizure from each patient is displayed.

Stage 1: Baseline: (Figure 3.3, column 1): The baseline, preictal stage was marked by diffuse, randomly distributed, low amplitude SI connections. No dominant spatial pattern was observed, though there were brief episodes of focality in the SOZ rarely persisting more than one second in duration.

Stage 2: Early Focality (Figure 3.3, column 2): Within a mean latency of 3.8\%T (SD 10.8, range (-34)-24) after seizure onset, focalities emerged with durations 


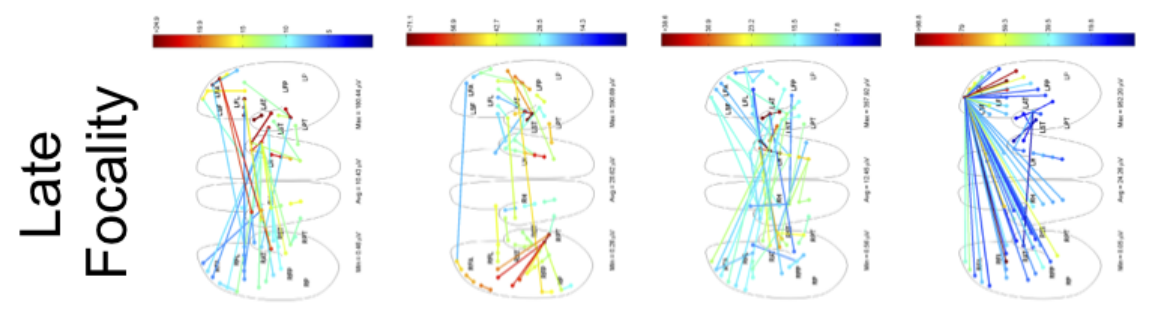

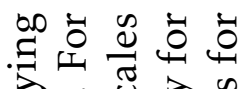

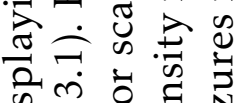
: \& $0 . \exists$ क

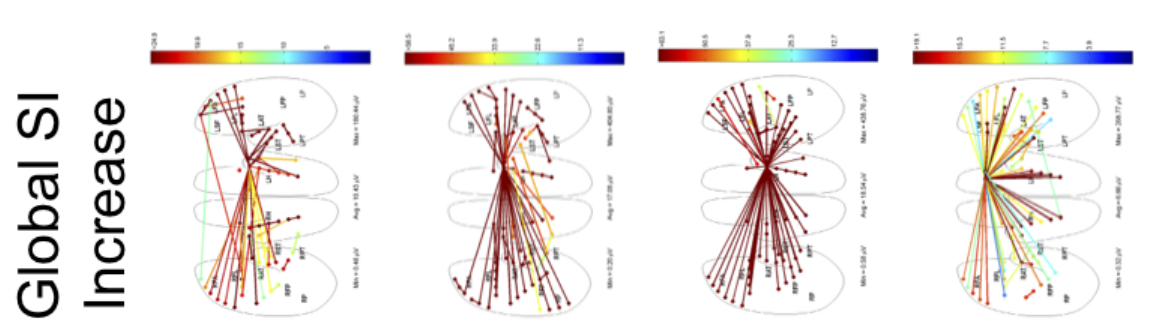
$\infty$

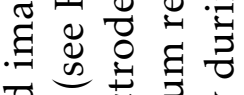

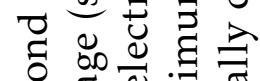
రิ

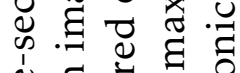

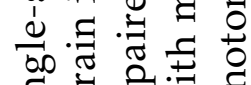

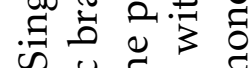

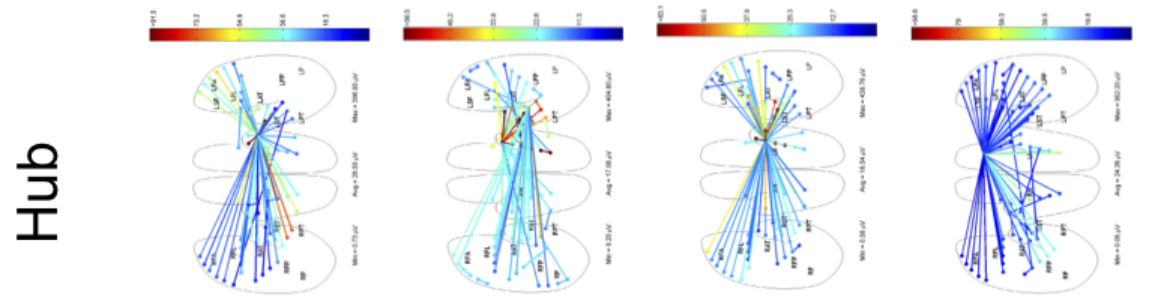

क.

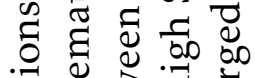

至窇

क : ปี ๘

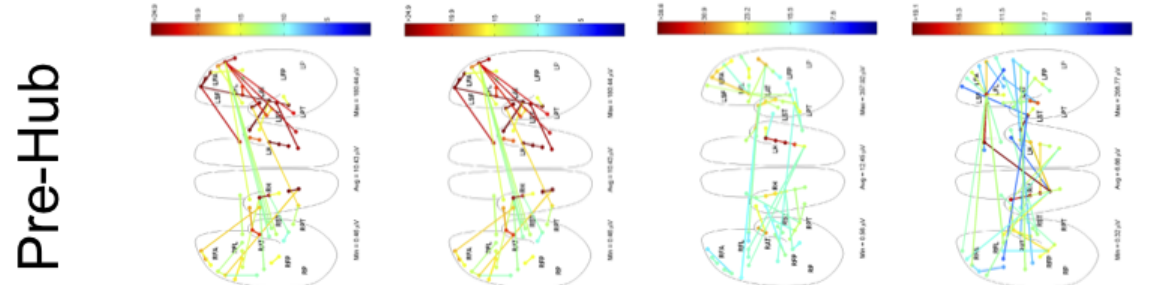

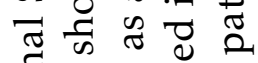

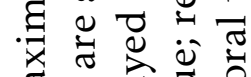

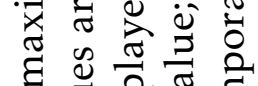
ते

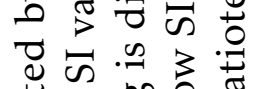
के $\dot{0}$ 范

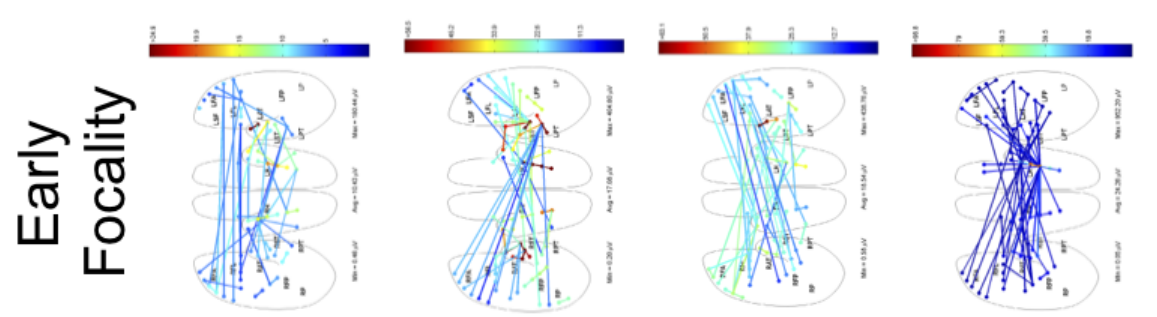
\&

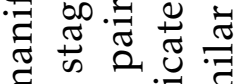
घ

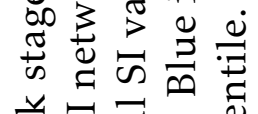
चै 3

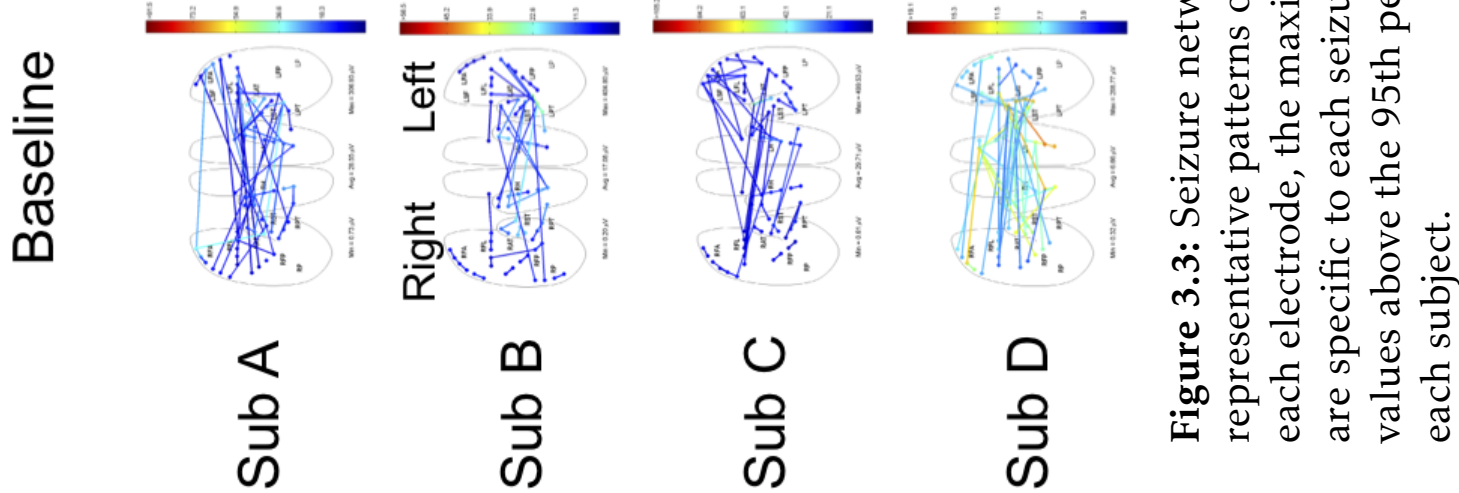


between $2-4$ seconds. The majority was located in cortical regions not within the clinically determined SOZ. These occurred on an unchanged background of low SI values elsewhere across the array and the connectivity pattern remained disorganized. This stage lasted a mean of $10.6 \% \mathrm{~T}$ (SD 11.7, range 2-48).

Stage 3: Pre-hub (Figure 3.3, column 3): This stage was often characterized by a shift in the location of the focality to electrodes within the SOZ. Typically as the second focality emerged the initial focality attenuated. Despite this change in focality, the baseline pattern persisted across the rest of the electrode array. This stage began at a mean of $14.8 \% \mathrm{~T}$ (SD 10.2, range 4-38) and lasted a mean of $7.6 \% \mathrm{~T}$ (SD 5.1, range 2-17).

Stage 4: Hub (Figure 3.3, column 4): The random spatial connectivity pattern seen in earlier stages abruptly coalesced to a dramatic hub pattern, with nearly all electrodes sharing their maximal SI connection to a single electrode that had been part of the pre-hub focality. The hub emerged at a mean latency of $22.3 \% \mathrm{~T}$ (SD 11.5 , range 9-49) and dominated the array for an average of $25.7 \% \mathrm{~T}$ (SD 12.5, range 3-67). In $22 / 25$ seizures, the hub center corresponded to the SOZ (correlations detailed below).

Stage 5: Global SI Increase (Figure 3.3, column 5): This stage manifested as an abrupt increase in SI values across the entire array (mean latency $46.8 \% \mathrm{~T}$ (SD 20.5, range 12-98)), such that nearly every electrode simultaneously surpassed the 95th percentile threshold. Despite this marked increase in SI values, the spatial connectivity pattern - the hub-remained unchanged. The duration of this stage comprised the majority of each seizure (49.5\% T (SD 18.6, range 14-79)).

Stage 6: Late Focality (Figure 3.3, column 6): Near seizure termination (mean latency 96.0\% (SD 17.4, range 37-115); duration 19.6\% (SD 14.6, range 1-62)), the spatial connectivity pattern again became diffuse, and the SI values of the 
majority of the electrode connections decreased to nearly baseline levels, leaving focality primarily in the electrodes central to the preceding seizure hubs and/or Early Focality.

The durations and sequences of SI connectivity stages were highly consistent within and among patients with a few exceptions. Five seizures (25\%) lacked just a single stage. Absence of a stage did not correlate with clinical or EEG characteristics. Two seizures (the last seizures from subject B) displayed spatial hub patterns but had small overall SI amplitude ranges and thus clear stage transitions could not be visualized. These atypical seizures were omitted from further analysis.

Duration (\%T) of each network stage was similar across subjects (Figure 3.4). The Early Focality stage (stage 2) was significantly longer for subject D than for subject $\mathrm{C}(\mathrm{p}<0.05)$, likely due to (a) 2 of this subjects 3 seizures had no discernible Pre-Hub stage (stage 3), such that the Early Focality lasted until the emergence of the Hub stage (stage 4) and (b) in one seizure, the Early Focality stage began nearly a minute before time zero.

\subsubsection{SI characteristics}

For each subject, nMaxSI values averaged across all electrodes showed a characteristic temporal profile with lowest values during the baseline stage, rising through Pre-Hub and Hub stages (stages 3-4), peaking at the Global Maximal SI stage (stage 5), and dropping as Late Focality occurred (Figure 3.5). These average nMaxSI differences by stage were statistically significant $(\mathrm{p}=0.002)$. Analysis of individual electrodes global average nMaxSI across seizures and subjects identified highest values at sites in the hippocampus and lateral temporal cortex (Figure 3.6a). This same distribution was confirmed in all individual seizures (Figure 3.6b). These electrodes were identical to those central to the focalities and hub seen during 


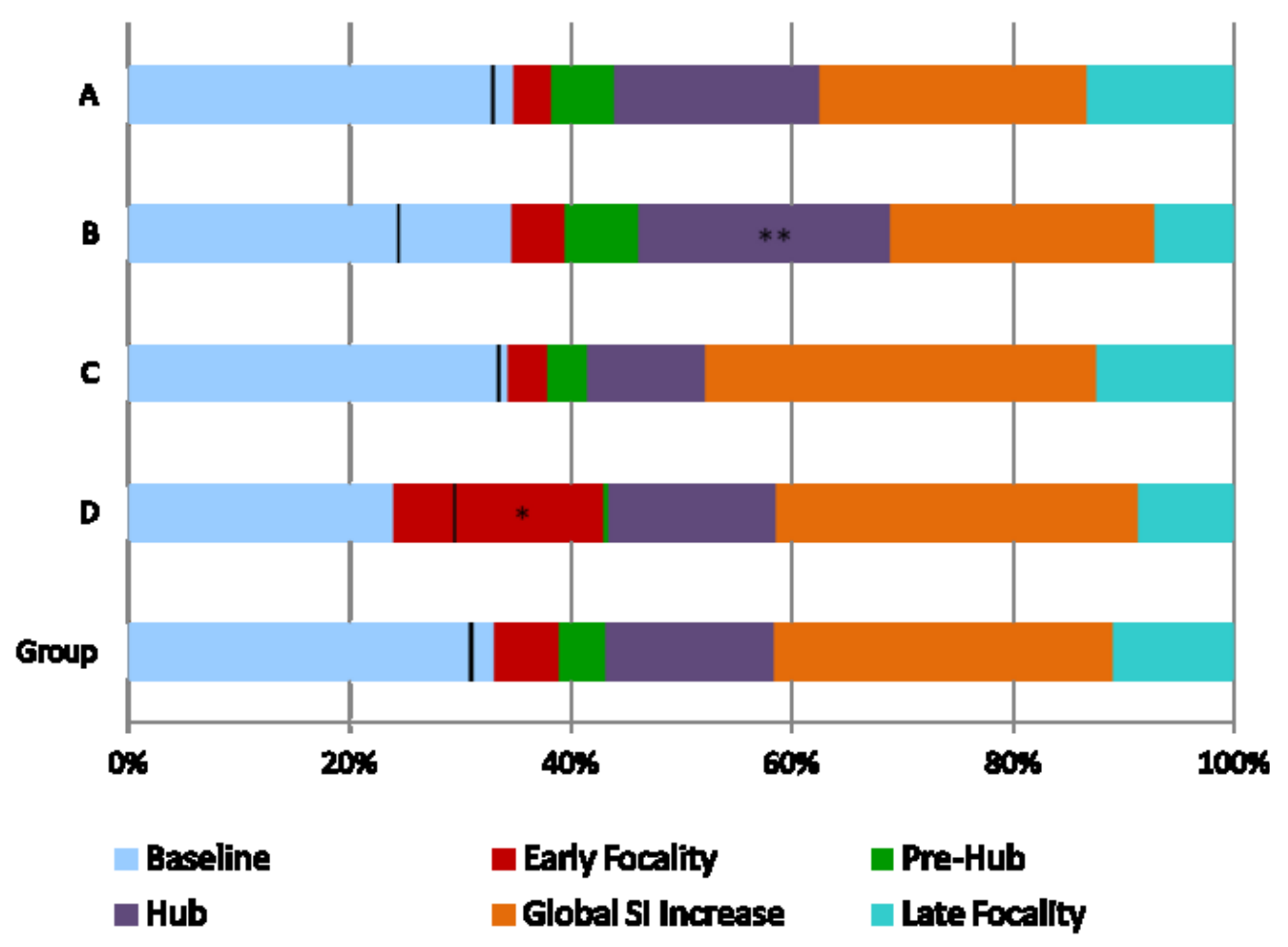

Figure 3.4: Relative Durations of Network Stages During Seizures. For each subject and for all subjects together (Group), average relative durations of each SI network stage are displayed as percentage of seizure time. For subject D, the Pre-Hub stage was not evident in 2 of 3 total seizures, resulting in prolongation of Early Focality. Additionally, in one of the seizures for that subject, Early Focality began well prior to visual seizure onset, increasing the average duration of that stage. Average Early Focality for subject D was longer than for subject $C(* \mathrm{p}<0.05)$. Seizure Hub was significantly longer in subject $\mathrm{B}$ than subject $\mathrm{D}(* * \mathrm{p}<0.05)$. Black lines indicate electrographic seizure onset by visual analysis. Overall, relative stage durations were remarkably similar across subjects.

connectivity animations, and were within the SOZ and resected region in 3 of 4 subjects. Conversely, electrodes in the contralateral frontal lobe, far from the SOZ, had significantly lower global average nMaxSI than the rest of the array $(\mathrm{p}=0.05)$.

Evaluation of average nMaxSI of individual electrodes during network stages revealed characteristic spatiotemporal patterns during seizures (Figure 3.6c). During Baseline and Early Focality (stages 1-2), lateral temporal electrodes showed highest 


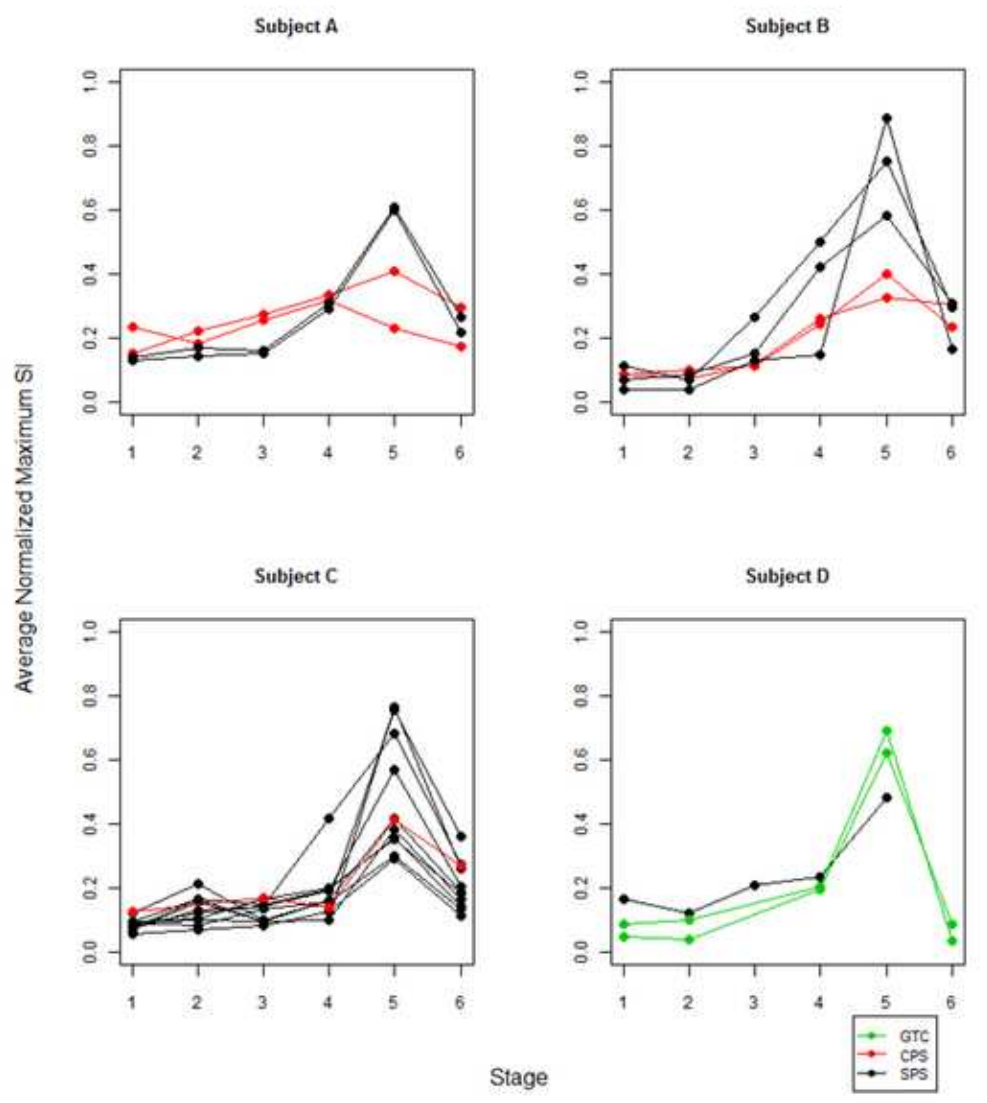

Figure 3.5: Normalized average SI values for each seizure across network stages. For all seizures from each subject, the normalized average maximum SI value across all electrodes is displayed for each network stage. Clinical severity of seizures is indicated by color (SPS=simple partial, CPS=Complex partial, GTC=secondary generalized tonic-clonic seizure). Temporal pattern of average SI value was similar for behaviorally similar seizures both within and across subjects. CPS had higher SI values than SPS during Global SI Increase (stage 5) $(\mathrm{p}<0.001)$.

nMaxSI (not significant). During Pre-Hub and Hub stages (stages 3-4), ipsilateral hippocampal electrodes had significantly elevated nMaxSI. Lateral temporal electrodes re-emerged with highest nMaxSI during the Global Increased SI stage (stage 5) and remained elevated through Late Focality (stage 6) while nMaxSI declined in the hippocampal electrodes (Table 3.2). Of note, for subject B, average nMaxSI of right hippocampal electrodes, identified as the SOZ by standard visual analysis of 

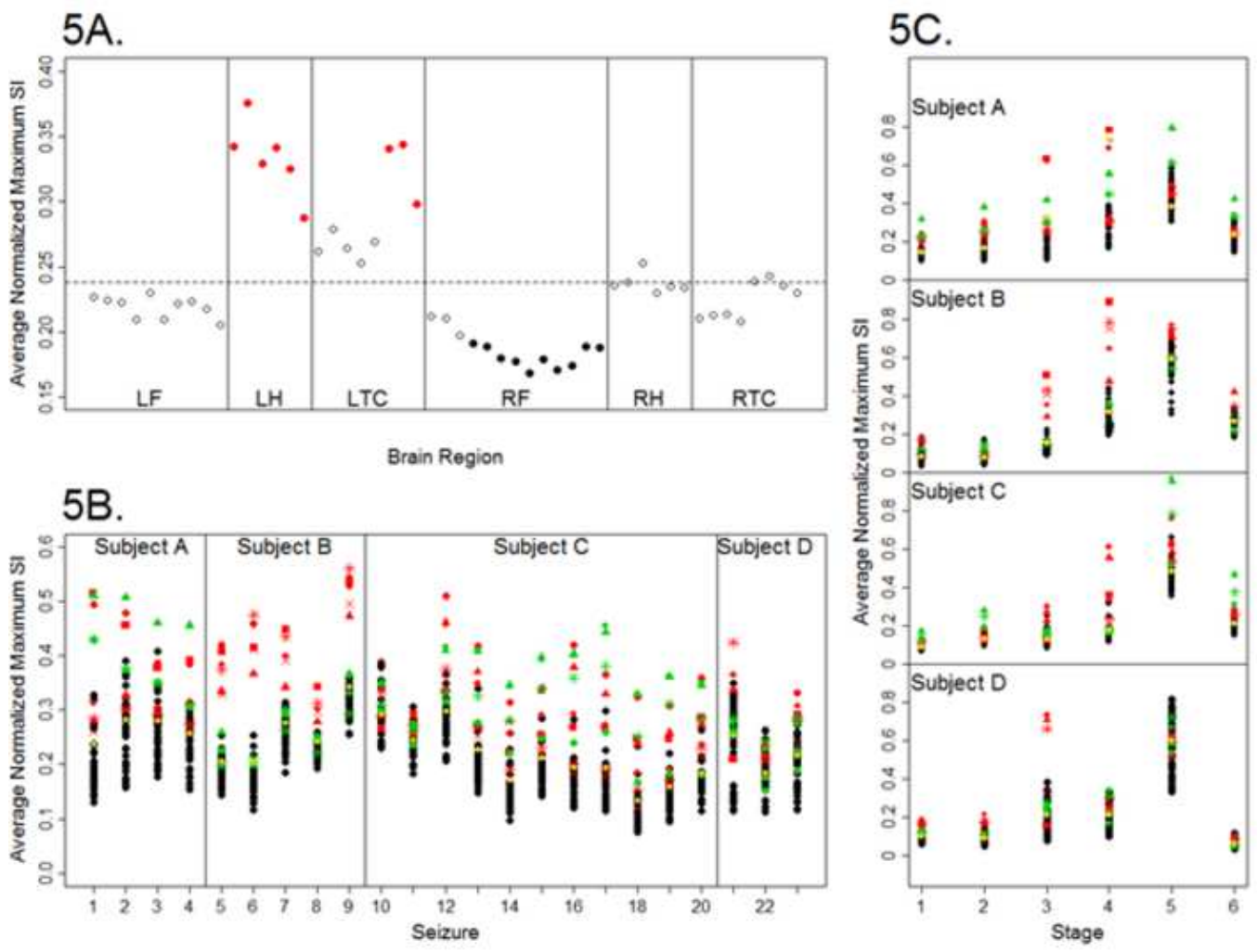

Figure 3.6: Hippocampal and Temporal Cortical Electrodes SI value distributions during Seizures. Electrodes with highest average nMaxSI correlated well with area of seizure manifestation by visual analysis. Electrodes identified at the group level were at the top of the distribution for each individual seizure. Stage analysis revealed that lateral temporal cortex dominates the early and late portions of seizures, while the hippocampus had highest connectivity during the hub stages. (5A) Least square means for each electrode across all subjects, seizures, and network stages. Dashed horizontal line represents the global average nMaxSI. Red dots show electrodes with significantly increased SI value, while black dots show those with significantly reduced SI value (adjusted $\mathrm{p}<0.05$ ). $\mathrm{L}=$ left, $\mathrm{R}=$ right, F: Frontal, H: Hippocampus, TC: Temporal Cortex. (5B) Average SI value of ipsilateral hippocampal (red) and temporal neocortical (green) electrodes shows them consistently at the top of the distribution for all analyzed seizures. Mean SI value for each seizure in gold. (5C) Average nMaxSI across seizures for each electrode at each network stage is plotted for each subject. Hippocampal electrodes (red) show higher connectivity during the hub stages ( 3 and 4 ), then recede as seizures terminate. Conversely, lateral temporal electrodes (green) dominate at the onset and termination of seizures (stages 1, 2, 5, 6) with less variability across stages. 
Table 3.2: Electrodes with higher SI value by stage and subject. Rows indicate all electrodes that displayed significantly elevated nMaxSI during any stage for any subject. Letters A-C signify individual subjects; "Group" indicates mean nMaxSI across subjects. All p-values were $<0.01$ (adjusting for multiple comparisons) unless otherwise noted. There were no significant electrodes during the Baseline or Early Focality stages. High SI values for individual electrodes indicate these electrodes mediated disproportionately strong connectivity during that seizure stage. Note the transition from elevated hippocampal electrodes (LOD) during Hub stages to lateral temporal electrodes (LTA, LST) during Global SI and late Focality stages. (See Figure 3.1 for electrode labels).

\begin{tabular}{ccccc}
\hline Electrode & Pre-Hub & Seizure Hub & Global SI & Late Focality \\
\hline LOD01 & A, Group & A,B,C, Group & & \\
LOD02 & A, Group & A,B,C, Group & C, Group & \\
LOD03 & & C, Group & & \\
LOD04 & & B, Group & & \\
LOD05 & B, Group & & \\
LOD06 & B & & \\
LTA02 & & A,C, Group & C, Group* \\
LTA03 & & A,C, Group & C \\
LTA03 & A & C, Group & \\
LTA03 & & & \\
\hline
\end{tabular}

${ }^{*}$ Indicates $0.05>\mathrm{p}$-value $>0.01$

EEG, never significantly differed from the mean.

The significant elevation and characteristic temporal behavior of nMaxSI of electrodes within the ipsilateral hippocampus and lateral temporal was a property found not only among individual seizures, but also consistently among pooled seizures within individual subjects and across pooled subjects (Table 3.2). The sole exception was subject D, for whom hippocampal electrode pairs showed trends towards higher values but were not significantly elevated above the theoretical average electrode values (likely because only 3 seizures were recorded). 


\subsubsection{Clinical Correlations}

\section{SOZ and surgical outcomes}

As demonstrated above, nMaxSI and the electrode location of seizure hubs corresponded to the precise electrodes of the SOZ and subsequent surgical location in 3 of 4 subjects. The one subject in whom nMaxSI did not identify the SOZ was Subject B. This subject had brief, focal EEG onset within the right hippocampus with rapid spread and subsequent evolution mainly expressed in the left hippocampus. The SI network topology revealed prominent left hippocampal connectivity in pre-hub and hub stages (stages 3-4) (Table 3.2). This subject underwent right-sided resection, and was not seizure-free two years after surgery. In this case of poor outcome, the lack of correspondence between visual analysis and SI methods may have predicted subsequent seizure recurrence.

Potential for prediction of surgical outcome was further suggested by the finding that, global average nMaxSI was significantly lower among patients with Engel Class 1a than those with Class $1 \mathrm{~d}(\mathrm{p}=0.018)$. Stage-level analysis demonstrated that this finding was driven by significantly higher SI values $(\mathrm{p}<0.0001)$ during the Seizure Hub stage (stage 4) in subjects with continued post-operative seizures (Figure 3.7).

\section{Seizure Severity and State}

Seizures with impaired consciousness (CPS) had higher nMaxSI during the Globally Increased SI stage (stage 5) than did seizures with retained consciousness (SPS) $(\mathrm{p}<0.001)$ (Figure 3.5). Seizures arising from wake did not differ in SI characteristics from those arising from sleep. Notably, baseline nMaxSI of electrodes within and outside the SOZ did not differ by sleep-wake state. 


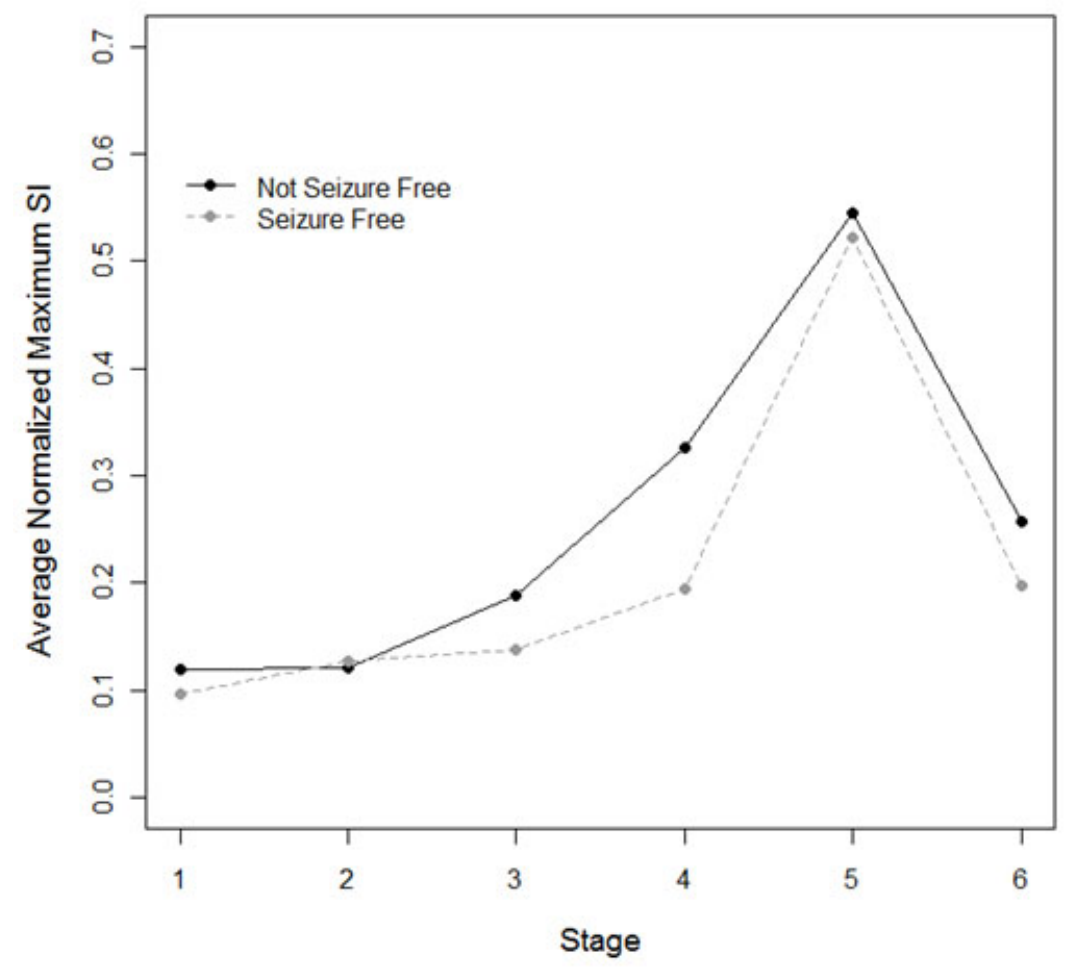

Figure 3.7: Average SI value at Seizure Hub is lower among subjects with post-surgical seizure freedom. Average nMaxSI plotted by network stage for subjects with Engel Class 1a vs. 1d. Average SI values during the Hub (stage 4) were significantly lower in patients who became seizure free after anterior temporal lobectomy $(\mathrm{p}=0.0001)$ than in those with recurrent seizures.

\section{Other patient characteristics}

No SI network properties differed by gender $(\mathrm{p}=0.699)$ or side of resection $(\mathrm{p}=0.231)$.

\subsection{Discussion}

This validation study of a measure of network connectivity, the Synchrony Index, undertaken on a highly selected sample of subjects with MTLE, demonstrated several important properties. 1) The methodology of connectivity animations 
effectively displayed complex connectivity data allowing the description of distinct stages of network synchrony corresponding to seizure initiation, propagation, and termination. 2) The measure of normalized maximum SI, calculated iteratively among all electrode pairs, corresponded to traditional EEG seizure onset localization. Exceptions appeared to correlate with failure of seizure remission after surgery. 3) The SI measure correlated with the severity of seizures, with greater amplitudes of synchrony indicating greater impairment of consciousness. 4) The SI measure correlated with surgical outcome, demonstrating higher values among patients who did not become seizure free.

An important limitation to the above initial findings is the small sample of highly selected patients; findings, of course, need to be examined with larger samples to determine sensitivity and specificity of SI measures in association with clinical factors. Although the subject number is small, strict inclusion criteria for purposes of validation is appropriate at this early stage. Not only were patients highly uniform in having unilateral MTLE with histopathologically confirmed HS, but electrode locations were broadly distributed (bilateral frontal, temporal, and hippocampal) and highly similar among patients. Further network characterization of classic MTLE may enable us to better distinguish it from its variants (secondary MTLE or dual pathology $[64,65]$ and other types of epilepsy. In addition, 4 patients and 25 seizures, compared to similar validation studies $[49,50,54,66,67]$, is a relatively comprehensive sample for these types of explorations.

The most important observation from this analysis of emergent spatiotemporal SI-derived ictal network patterns was the consistency and reproducibility within a homogenous group of seizures from subjects with unilateral MTLE. These patterns correlated well with seizure localization by traditional visual analysis of intracranial EEG. These results support SI-derived connectivity animations as a valid method 
for evaluation of network behavior during temporal lobe seizures at the group level. The strength of SI network quantification appears to be in the concordances among the Hub stage (stage 4) of seizures (indicating a network of tightly coupled electrodes centered on the anterior hippocampus, traditional EEG localization of seizure onset, and subsequent outcome of temporal lobectomy. For example, in 3 out of 4 of patients, the location of the "hub" stage correlated with visual seizure localization.

SI networks did not appear especially useful in prediction of seizure initiation. In fact, the SI hub stage (the most useful localizing finding, and the most visually striking during connectivity animations) occurred with a typical latency of 10-15 seconds after traditional EEG seizure onset. In contrast, SI networks appeared to be a powerful visual and quantifiable marker of maximal seizure propagation. Based on the subject with discordance between location of brief seizure onset and the contralateral location of the SI hub (subject B), one could hypothesize that surgery targeting the traditional SOZ may not yield seizure remission when the location of visual seizure initiation is distinct from that of maximal SI propagation. Discordance between onset and propagation have analogues in earlier studies of traditional intracranial EEG that showed the rapidity of contralateral spread after focal initiation correlated with poor seizure remission [68].

Another feature of SI networks during limbic seizures that we found intriguing was that the visual appearance of a hub—centered within the hippocampus and closely coupled to ipsilateral temporal neocortex-is a striking mathematical support of the concept of epileptic networks [5, 44]. Furthermore, the magnitude of the SI may reflect severity or extent of pathology and susceptibility of the network to disruption by surgical intervention, as suggested by the correlation between higher SI values and post-surgical seizure recurrence. 
Some network features were surprising; early SI focality in ipsilateral neocortical temporal electrodes preceded high SI values in the hippocampal SOZ. This finding is not unprecedented; extrahippocampal atrophy as an anatomic correlate to connectivity networks is common in refractory MTLE $[69,70]$. Seizure onsets in the parahippocampal cortex [6] and entorhinal cortex [71] have been reported. These combined findings support the hypothesis that "limbic seizures" may require a network interaction for initiation $[5,72,73]$, and highlight the importance of developing improved techniques for network characterization.

\subsection{Conclusion}

This chapter presents a novel approach for display of ictal network dynamics utilizing the Synchrony Index as a measure of inter-electrode connectivity. This technique accurately identifies the area of seizure manifestation, as determined by standard visual analysis and confirmed by two years of post-operative follow-up. The emergent spatiotemporal patterns demonstrated by this method showed intraand inter-subject consistency at both the network and electrode-specific level, while distinguishing clinically relevant features such as seizure severity and surgical outcome. 


\section{Chapter 4}

\section{Synchronization of Neural Action Potentials Using Global Feedback ${ }^{1}$}

\subsection{Introduction}

Abnormal synchronization of neural activity can be seen in many neurological diseases including epilepsy, Parkinsons disease and essential tremors [74-77]. Neurostimulation therapy can be used to alleviate the symptoms of these diseases $[10,11]$; it typically involves applying a pulse-train stimulation signal to an electrode which has been surgically implanted into the brain of the patient $[8,12,78]$. This electrical stimulation signal modulates the extracellular potential of all of the neurons within the targeted area, which is thought to alter their collective behavior. One main challenge is determining the necessary stimulation parameters in order to obtain the desired collective firing behavior. While electrical stimulation has been shown to modulate the activity of individual neurons, its effect on the synchronization behavior of a group of neurons is currently under investigation

\footnotetext{
${ }^{1}$ C.G. Rusin, S.E. Johnson, J. Kapur and J.L. Hudson, "Engineering the Synchronization of Neuron Action Potentials Using Global Time-delayed Feedback Stimulation" Phys. Rev. E, 84, 066202, 2011.
} 
$[79,80]$. Theoretical models have been developed which illustrate how electrical stimulation can be utilized to alter the firing patterns of simulated populations of neurons $[14,81]$.

Time-delayed feedback has been shown to desynchronize groups of model neurons $[14,82-86]$, and can be employed in place of pulse-train stimulation. The feedback can be used to design a therapeutic state of synchronization $[87,88]$. The application of locally-addressable stimulation has been shown to synchronize the action potentials of neurons [89].

This chapter experimentally demonstrates the use of time-delayed feedback stimulation for engineering the synchronization of the action potentials of cultured neurons. Phase models have been shown to have sufficient accuracy to allow precise control over synchronization states of complex oscillatory systems [39, 89-96]. The standard approach for the construction of a phase model involves measuring the phase response of a system to a set of discrete pulses applied at specific times in the cycle of the element $[89,92,97-102]$. However, precise measurements of phase shift are difficult to obtain, particularly when, for example, the period is nonstationary. In this chapter, an experimental method was used to construct phase based models using continuous stimulation of a single patch clamped neuron. This method overcomes some of the limitations inherent in standard pulse-based approaches. The mean period of the neuron was measured with and without applied feedback. The change in the mean period of the action potentials as a result of the application of feedback was determined as a function of feedback delay. This allowed for the construction of a phase model of the dynamical behavior of the neuron. The experimentally constructed model was then used to determine the feedback parameters necessary to produce in-phase and anti-phase synchronization states within a two neuron system using global stimulation. The parameters were 
applied to an experimental system of two patch clamped neurons, and the desired synchronization states were observed. The validated model was then used to determine feedback parameters which may disrupt the synchronization of a large population of globally coupled neurons.

\subsection{Background}

Phase models are useful tools for describing the dynamics of complex oscillating systems $[90,103,104]$. Instead of describing the activity of chemicals and potentials to model a neuron, the behavior of the neuron can be described using the phase in a phase model.

A key component of the phase model is the response curve which describes the response of the oscillator for any stimulation. Phase response curves (PRCs) have been measured for many different types of neurons such as cortical $[98,101]$, mitral cells [92], stellate cells [99] and abdominal ganglion [100, 105]. PRCs can be used to model larger neural networks $[97,98,102,106,107]$. Similar functions that measure the response of a neuron to perturbations have been reported, such as the spike time response curve (STRC) [89].

The PRC can be measured experimentally by the input of pulses at various phases of the neuron's cycle and the advance or delay of the next peak is recorded $[89,92$, 97-102]. This method of measuring the PRC from neurons can be difficult since the timing of the subsequent peak (action potential) is in itself variable. To adjust for the natural variability in the period of the cells, Netoff et al. applied a rate controller that raises or lowers the membrane potential to increase or decrease the period of the neuron, resulting in a constant period $[89,99]$. Recently, PRCs have been measured using a fluctuating applied current, where weighting is used to correct 
for a variable period [108]. Another method of measuring a PRC was suggested by Cui et al. [105] where the neurons received a perturbation for every cycle until a steady state was achieved. Predictions of action potential synchronization for systems of coupled neurons have been generally related to excitatory or inhibitory activity $[89,99,102,106]$; however, any type of stimulation can be used to control the behavior of oscillating elements [90].

\subsection{Theory}

A phase based model can be constructed to represent the dynamical behavior of a population of coupled oscillatory elements

$$
\frac{\mathrm{d} \phi_{i}}{\mathrm{~d} t}=\omega_{i}+\frac{K}{N} \sum_{j=1}^{N} H\left(\phi_{j}-\phi_{i}\right) \quad \text { for } i=1,2, \ldots, N
$$

where $\phi_{i}$ is the phase of the element, $K$ is the interaction strength, $\omega_{i}$ is the natural frequency, and $H(\Delta \phi)$ is the interaction function $[109,110]$. The interaction function can be determined from macroscopic physical quantities

$$
H(\Delta \phi)=\frac{1}{2 \pi} \int_{0}^{2 \pi} Z(\phi) h(\phi+\Delta \phi) \mathrm{d} \phi
$$

where $Z(\phi)$ is the response function and $h(\phi)$ is the stimulation function [110]. The response function quantifies the sensitivity of the neuron to perturbations as a function of phase, while the stimulation function quantifies the amount of stimulation applied to the neuron at a given phase.

The construction of the model proceeds by experimental determination of the response function of the neuron. Standard methods for determining the response 
function of an oscillatory element often require large pulses [111] or access to multiple coupled elements [112], making them difficult to apply in experimental systems. As a result, a method was developed which utilized weak delayed selffeedback stimulation applied to a single oscillator [113]. A phase model can be defined for a system composed of 1 element as

$$
\frac{\mathrm{d} \phi_{1}}{\mathrm{~d} t}=\omega_{1}+K H\left(\phi_{1}^{\dagger}-\phi_{1}\right)
$$

where $\phi_{1}^{+}$is the phase of the stimulation signal applied to the element. For delayed self-feedback stimulation, the phase of the stimulation is related to the phase of the physical element by

$$
\phi_{1}^{\dagger}=\phi_{1}-\tau
$$

where $\tau$ is the feedback delay.

The phase of an oscillator can be integrated over one cycle and expressed as a function of the period of the oscillation

$$
2 \pi=\oint \mathrm{d} \phi_{i}=\int_{0}^{P_{i}+\Delta P_{i}} \mathrm{~d} t \frac{\mathrm{d} \phi_{i}}{\mathrm{~d} t}
$$

where $P_{i}$ is the intrinsic mean period of the oscillator $\left(2 \pi / \omega_{i}\right)$, and $\Delta P_{i}$ is the change in the period of the oscillator due to external stimulations, such that $P_{i}+\Delta P_{i}$ is the observed period of the element $[112,114]$. Substituting equation 4.3 and 4.4 into 4.5, $H(\Delta \phi)$ can be analytically approximated as

$$
\begin{aligned}
H(\Delta \phi) & =\frac{-2 \pi}{K P_{1}^{2}}\left[\Delta P_{1}(\Delta \phi)\right] \\
\Delta \phi & =-\tau .
\end{aligned}
$$


Equation 4.6 allows for the determination of the interaction function directly from experimental measurements of the period of a oscillatory element stimulated using delayed self-feedback. A similar method was developed using a synaptic stimulation function by Cui et al. [105]. This method of using one oscillator to develop a phase model has also been tested using electrochemical oscillators [115].

$Z(\phi)$ can be analytically determined from equation 4.2 given an experimentally measured interaction function obtained under a known stimulation. Each function in equation 4.2 can be expanded in a Fourier series

$$
\begin{aligned}
H(\Delta \phi) & =\sum_{n=1}^{\infty} R_{n} \cos (n \Delta \phi)+S_{n} \sin (n \Delta \phi) \\
Z(\phi) & =\sum_{m=1}^{\infty} A_{m} \cos (m \phi)+B_{m} \sin (m \phi) \\
h(\phi) & =\sum_{l=1}^{\infty} C_{l} \cos (l \phi)+D_{l} \sin (l \phi) .
\end{aligned}
$$

Substituting these Fourier series into equation 4.2 and integrating, yields a linear system of equations in terms of their Fourier coefficients

$$
\left(\begin{array}{cc}
C_{n} & D_{n} \\
D_{n} & -C_{n}
\end{array}\right)\left(\begin{array}{c}
A_{n} \\
B_{n}
\end{array}\right)=\left(\begin{array}{c}
2 R_{n} \\
2 S_{n}
\end{array}\right)
$$

which can be solved using standard matrix techniques.

Once the response function is known, Equation $4.1 \& 4.2$ can be used to determine how the the parameters of the stimulation function affect the collective phase behavior of a set of two or more neurons. For a system of two neurons, we construct 
a phase model of the form

$$
\frac{\mathrm{d} \phi_{i}}{\mathrm{~d} t}=\omega_{i}+\frac{K}{2} \sum_{j=1}^{2} H\left(\phi_{j}-\phi_{i}\right) \quad \text { for } i=1,2
$$

which by subtraction yields

$$
\frac{\mathrm{d} \Delta \phi}{\mathrm{d} t}=\Delta \omega+\frac{K}{2}[H(-\Delta \phi)-H(\Delta \phi)]
$$

Stationary solutions to this equation will occur at phase differences which satisfy

$$
\frac{\Delta \omega}{K}=H^{-}(\Delta \phi)
$$

where $H^{-}(\Delta \phi)$ is the odd part of the interaction function. A linear stability analysis indicates that these stationary states will be stable when

$$
\frac{\mathrm{d} H^{-}(\Delta \phi)}{\mathrm{d} \Delta \phi}>0
$$

Synchronization states can therefore be generated by selecting feedback stimulation parameters such that the interaction function has the necessary properties (Equation $4.13 \& 4.14)$ to stabilize the desired states.

\subsection{Methods}

\subsubsection{Cultures}

Cultures were prepared from P0-P1 Sprague-Dawley newborn rats using methods similar to those described elsewhere $[116,117]$. The newborn rats were decapitated, their brains removed and placed in cold HEPES-buffered Hank's balanced 
salt solution (HEPES-HBSS). The hippocampi were removed under a dissecting microscope and collected in a small petri dish containing HEPES-HBSS. Tissues were incubated in $0.125 \%$ trypsin for $15 \mathrm{~min}$ at $37^{\circ} \mathrm{C}$. Trypsin solution was replaced with $5 \mathrm{~mL}$ HEPES-HBSS and the cells were rinsed twice more with HEPES-HBSS at 5 min intervals. Hippocampi were triturated until no fragments of tissue remained. Neurons were collected by centrifugation and resuspended in $5 \mathrm{~mL}$ of Dulbeccos modified Eagles medium (DMEM) and F-12 supplement (1:1) (Invitrogen) with $10 \%$ fetal bovine serum (heat-inactivated, Invitrogen), $2 \mathrm{mM}$ L-glutamine (Invitrogen), and penicillin $(100 \mathrm{U} / \mathrm{mL})$-streptomycin $(100 \mathrm{U} / \mathrm{mL})$. Culture dishes were coated with poly-lysine and filled with $2 \mathrm{~mL}$ of culture medium. Cells were plated at a minimum density of 50,000 per $35 \mathrm{~mm}^{2}$ dish and kept at $37^{\circ} \mathrm{C}$ in a $5 \% \mathrm{CO}_{2}$ incubator. After $24 \mathrm{~h}$, the culture medium was changed to serum-free medium containing $2 \%$ B 27 and $2 \mathrm{mmol} / \mathrm{L}$ glutamine. The medium was replaced with fresh medium every $2-3$ days.

\subsubsection{Electrophysiology}

Culture medium was discarded and replaced with an external medium consisting of (in $\mathrm{mM}$ ): $146 \mathrm{NaCl}, 3 \mathrm{KCl}, 2 \mathrm{CaCl} 2,3 \mathrm{MgCl} 2,11$ glucose, and 10 HEPES, $\mathrm{pH}$ 7.4, osmolarity 310-315 mOsm. The external medium was sterile filtered before use. The neurons were synaptically isolated by adding $50 \mu \mathrm{M}$ DL-2-Amino-5phosphonopentanoic acid (DL-AP5), $50 \mu \mathrm{M}$ bicuculine methiodide (BIC) and $20 \mu \mathrm{M}$ 7-Dinitroquinoxaline-2,3-dione (DNQX). DL-AP5 blocks NMDA receptors, DNQX blocks AMPA and Kainate receptors and BIC blocks GABA $_{A}$ receptors. In order to create repetitive periodic spiking, $50 \mu \mathrm{M}$ of 4 -aminopyridine (4AP) was added to the external solution.

Micropipettes were pulled on a Sutter Instrument Co. Flaming/Brown Mi- 


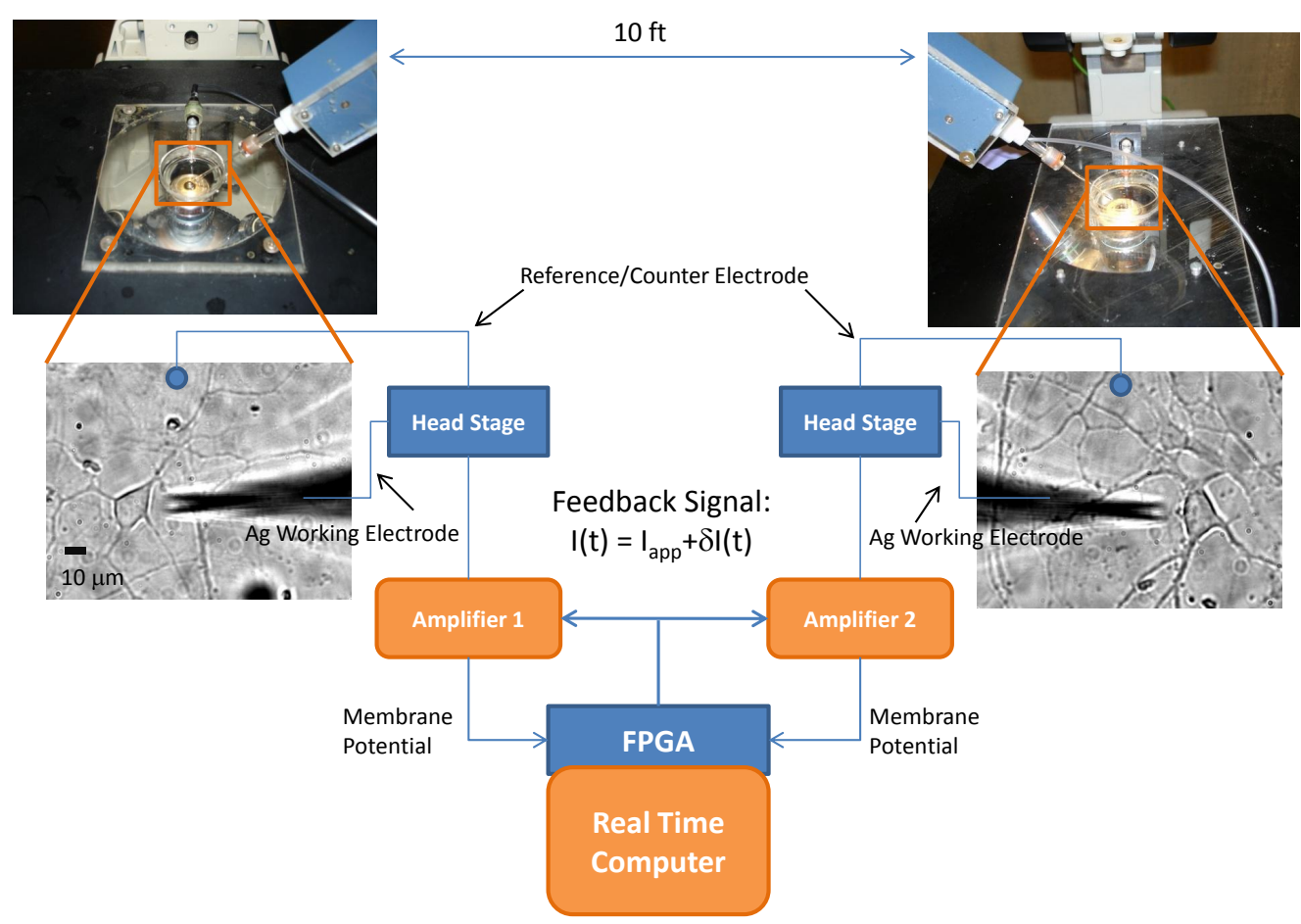

Figure 4.1: Schematic of the dynamic clamp system. For one cell experiments only one apparatus was used. For the two neuron experiments both apparatuses were used.

cropipette puller (Model P-97) from borosilicate glass (O.D. $1.5 \mathrm{~mm}$, I.D. $0.86 \mathrm{~mm}$ ) using a 1-stage pull protocol. Micropipettes with resistances of 6-9 $\mathrm{M} \Omega$ s were filled with a solution of (in mM): $145 \mathrm{~K}$-gluconate, 0.6 EGTA, $11 \mathrm{HEPES}, 8 \mathrm{KCl}, 3$ $\mathrm{NaCl}$ and $4 \mathrm{MgATP}, \mathrm{pH} 7.3$, osmolarity 295-300 mOsm. The internal solution was sterile filtered before use. $\mathrm{HEPES}$, K-gluconate, $\mathrm{KOH}, \mathrm{KCl}, \mathrm{MgCl}_{2}, 4-\mathrm{AP}, \mathrm{MgATP}$, EGTA and DMSO were from Sigma; glucose from Sigma-Aldrich; $\mathrm{NaCl}, \mathrm{CaCl}_{2}$ and $\mathrm{NaOH}$ were from Fischer Scientific; and DNQX, DL-AP5 and Bic were from TOCRIS bioscience.

After obtaining electrical access to the cell, the cell was hyperpolarized to -70 $\mathrm{mV}$ to prevent any undesired firing while the cell was resting. A constant baseline current was applied to the cell of sufficient amplitude to cause repetitive spiking for 
10 seconds, after which the cell was returned to resting for 20-30 seconds before the current was applied again. The baseline current was applied three times to get an accurate value for the mean period of the unperturbed membrane potential.

Linear feedback was added to the baseline current after one second. The stimulation was of the form

$$
\begin{aligned}
I(t) & =I_{a p p}+\delta I \\
\delta I & =\frac{K}{N} \sum_{i=1}^{N} h\left(V_{i}(t)\right) \\
h(V) & =K_{0}+K_{1}\left(V(t-\tau)-V_{m}\right)
\end{aligned}
$$

where $I(t)$ is the injected current stimulation signal, $I_{a p p}$ is the baseline injected current, $V_{m}$ is the mean value of the membrane potential, $K$ is the feedback gain, and $V$ is the measured neuron membrane potential as a function of time, $t$, and delay, $\tau$ (Figure 4.2 A \& B). The mean period of the unperturbed membrane potential was used to calculate the delay for the feedback. The feedback delay was incremented from 0 to 1 in 0.05 steps. At each feedback delay, the cell was depolarized for 10 seconds and then allowed to rest for 20-30 seconds. The cells were depolarized three more times (10 seconds applied, 20-30 seconds resting) to insure that the average baseline firing frequency had not been altered over the course of the experiment.

\subsubsection{Experimental Apparatus}

The program Neuro-Syncroscope 2008 was coded and the apparatus was designed by Craig G. Rusin, and more details can be found in his dissertation [115]. A schematic of the dynamic clamp system can be seen in Figure 4.1. Two antivibration tables were each equipped with a grounded metal mesh Faraday cage, mobile stage and a Nikon inverted microscope. The part of the apparatus designated to be cell 
1 had a SD Instruments micromanipulator, an Axopatch 200A amplifier and a Leader 8020 oscilloscope. The part of the apparatus for cell 2 had a Burleigh P5000 micromanipulator, an Axopatch 1-D amplifier and a Leader 1021 oscilloscope. One dish was placed on each microscope and a silver chloride reference/counter electrode was placed in each dish. Standard whole cell patch clamp technique was used to obtain electrical access to the neuron [118].

The amplifiers were connected to the DAQ system, which consists of a Labview real time computer and a Labview host computer. The real time computer contains a National Instruments PCI 7833R DAQ card with an integrated FPGA processor to allow for data acquisition and real time feedback calculations on the order of $25 \mathrm{kHz}$. Other similar dynamic clamp apparatuses have been discussed in the literature $[119,120]$. Each of the amplifiers had an internal analog output scaling factor; 1 for the Axopatch 200A and 10 for the Axopatch 1D. The adjustable analog output scaling factor, $\alpha$, was set to be 50 on the Axopatch 200A and 5 on the Axopatch 1D to ensure that the overall analog output scaling factor for both amplifiers was 50 . Voltage dividers were built to allow the resolution of the stimulation signal applied from the DAQ card through the amplifier to be on the order of $1 \mathrm{pA}$. The voltage dividers were fabricated to match the specific impedance of the amplifier to which they were attached.

\subsection{Results}

\subsubsection{Measurement of the Interaction Function}

Experiments were conducted to illustrate the use of global feedback stimulation for controlling the synchronization behavior of neurons. The interaction function of a single, synaptically isolated neuron to delayed feedback stimulation was ex- 
perimentally measured. A single neuron was patch clamped and its membrane potential was recorded over time (Figure 4.2 A). Delayed feedback stimulation was then applied to the neuron. Figure 4.2 B-D illustrates the firing period of the neuron as a function of feedback delay. Baseline observations of the natural firing rate of the neuron (without applied stimulation) were taken before and after each experiment (left and right panels). The natural period distribution of the neuron was observed to be approximately the same before and after the application of feedback stimulation, indicating that the stimulation did not disrupt the intrinsic behavior of the neuron. Experiments were repeated using three different neurons.

The period of the neuron action potentials was observed to change as the feedback delay was increased (middle panels, Figure 4.2 B-D). The maximum firing period was achieved with a feedback delay of approximately $\tau=0.25 \mathrm{rad} / 2 \pi$ while minimum firing period was obtained at approximately $\tau=0.7 \mathrm{rad} / 2 \pi$. The firing period of the neuron with $\tau=1 \mathrm{rad} / 2 \pi$ was close to the firing period when $\tau=0$ $\mathrm{rad} / 2 \pi$. Drift in the natural period of the action potentials occurred, but was small compared to the mean period of the action potentials, except for the case of the second cell (Figure 4.2 C). For this cell, the mean period was adjusted linearly from the initial pre-stim mean period to the post-stim mean period. The qualitative frequency response was found to be similar for each neuron.

Equation 4.6 was applied to the experimental observations in Figure 4.2 B-D; the resulting interaction functions are illustrated in Figure $4.3 \mathrm{~A}$. The period of the neuron action potentials was taken to be the median period of the observed action potentials. Once the interaction functions were determined for each of the neurons, their corresponding response functions were calculated using Equation 4.2. For this calculation, the stimulation function was set to Equation 4.17 and the neuron waveform, $x(\phi)$, was set to the mean cycle of the neuron action potential. 

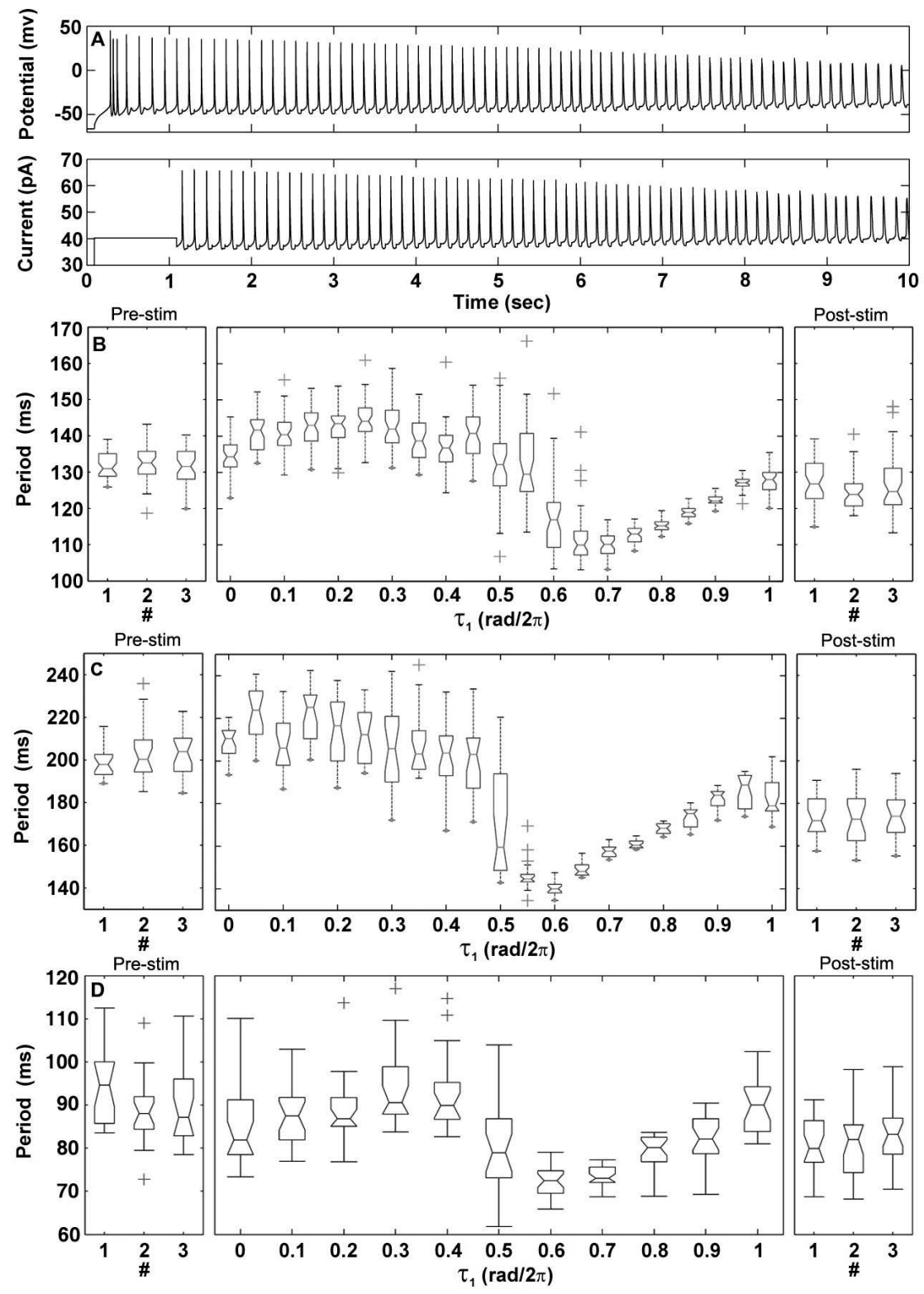

Figure 4.2: The interaction function is measured by applying self-delayed feedback to a single patch clamped neuron. A) The membrane potential of a single neuron (top) and the applied stimulation signal (bottom) as a function of time $\left.\left(K_{0}=13 \mathrm{mV}, K_{1}=350, \tau=0.5 \mathrm{rad} / 2 \pi\right) . \quad \mathrm{B}, \mathrm{C} \& \mathrm{D}\right)$ Period distributions for three separate cells. Middle panel shows the period distribution as feedback stimulation delay was increased from from 0 to $1 \mathrm{rad} / 2 \pi$. Left and right panels illustrate the period distribution of the neuron action potentials before and after application of stimulation. 

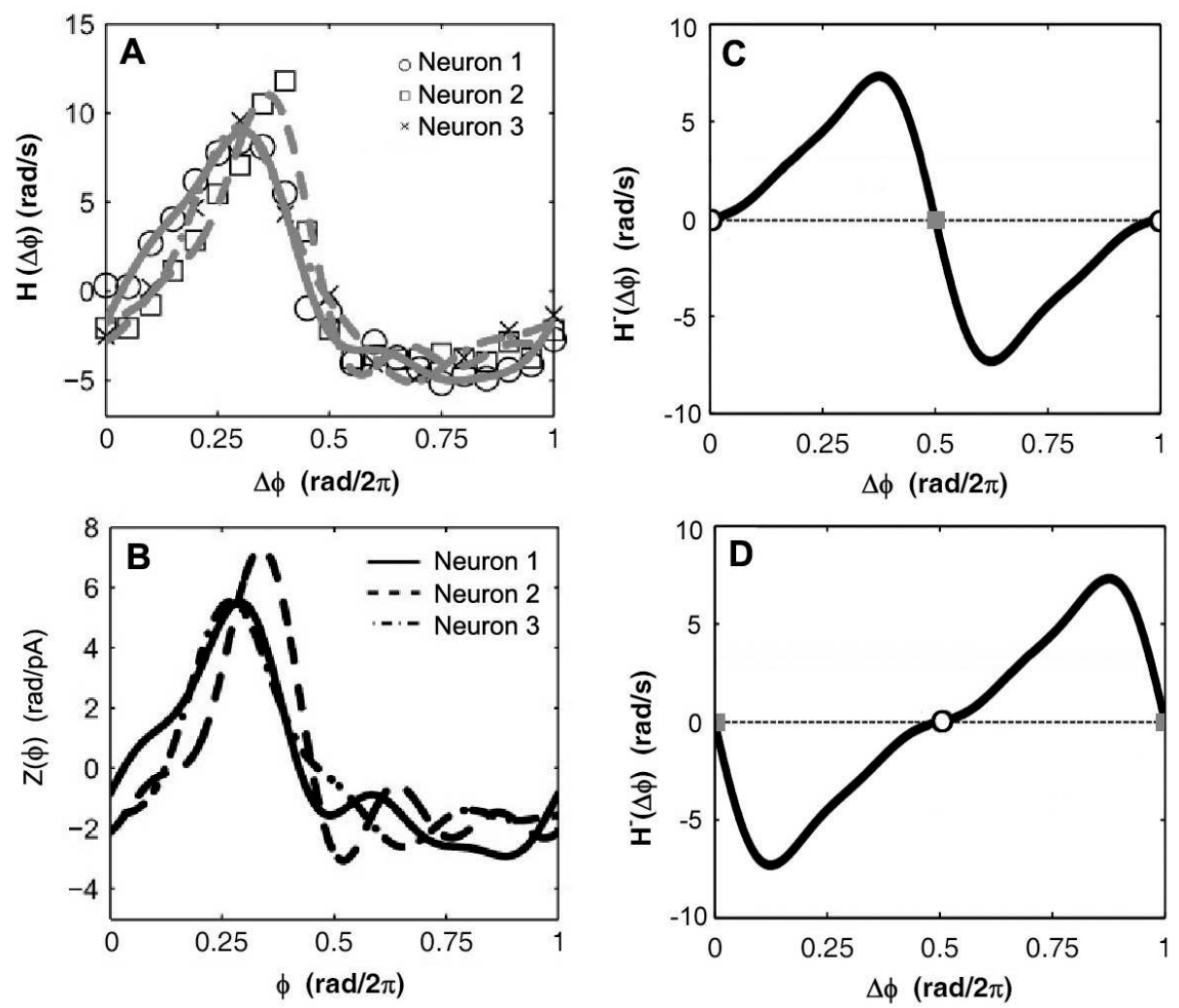

Figure 4.3: Experimentally measured interaction function and response functions for three different neurons predict and in-phase and anti-phase synchronization states for two coupled neurons. A) Calculated interaction function data and Fourier fit for the three neurons shown in Figure 4.2 B-D. B) Calculated response functions for the three isolated neurons. C) Odd part of the interaction function for the experimental system with applied global feedback $\left(K_{0}=13 \mathrm{mV}, K_{1}=350\right.$, and $\left.\tau=0 \mathrm{rad} / 2 \pi\right)$. D) Odd part of the interaction function for the experimental system with applied global feedback $\left(K_{0}=13 \mathrm{mV}, K_{1}=350\right.$, and $\left.\tau=0.5 \mathrm{rad} / 2 \pi\right)$. In C) and D) the open circles are stable states and the gray squares are unstable states.

The resulting response functions for each of the neurons are illustrated in Figure 4.3 B. Qualitative agreement was seen between these three functions, indicating that their sensitivities to electrical stimulation are approximately equal. 


\subsubsection{Synchronization of Two Neurons under Global Feedback}

The experimentally constructed phase model was used to determine the values of feedback delay which would produce in-phase and anti-phase synchronization states in a system of two neurons. As indicated by Equations 4.12 and 4.14, a system of two neurons will exhibit a stable phase-locked state when the odd part of the interaction function equals the value $\Delta \omega / K$ with positive slope. Assuming the ratio of $\Delta \omega / K$ is small, the stationary states of the system can be found at the roots of $H^{-}(\Delta \phi)$. The roots of $H^{-}(\Delta \phi)$ for the experimental system were determined as a function of the feedback delay. It was observed that feedback delays less than $0.2 \mathrm{rad} / 2 \pi$ produced a unique and stable in-phase synchronization state while feedback delays between 0.4 and $0.6 \mathrm{rad} / 2 \pi$ produced a unique and stable antiphase synchronized state. Figure 4.3 C \& D illustrates the odd part of the interaction function for feedback delay of 0 and $0.5 \mathrm{rad} / 2 \pi$ respectively.

The identified feedback delays were applied to the two neuron system in order to produce the expected in-phase and anti-phase synchronization states. To insure that the neurons were connected only through the feedback stimulation, the neurons were synaptically isolated and placed in separate cultures. Both neurons were patch clamped using standard methods. The recorded membrane potentials for both cells under different experimental conditions can be seen in Figure 4.4 A1-A3; the applied stimulation signal is illustrated in Figure 4.4 B1-B3. The neurons were observed to experience accommodation for the first three seconds of each experiment; this data was not considered as part of the analysis.

Without feedback stimulation, the phases of the action potentials of the two neurons were found to be uncorrelated (Figure 4.4 A1-E1). The mean periods of the cells were not observed to lock and no preferred phase orientation was found (Figure $4.4 \mathrm{E} 1$ ). Application of the global feedback stimulation with a 

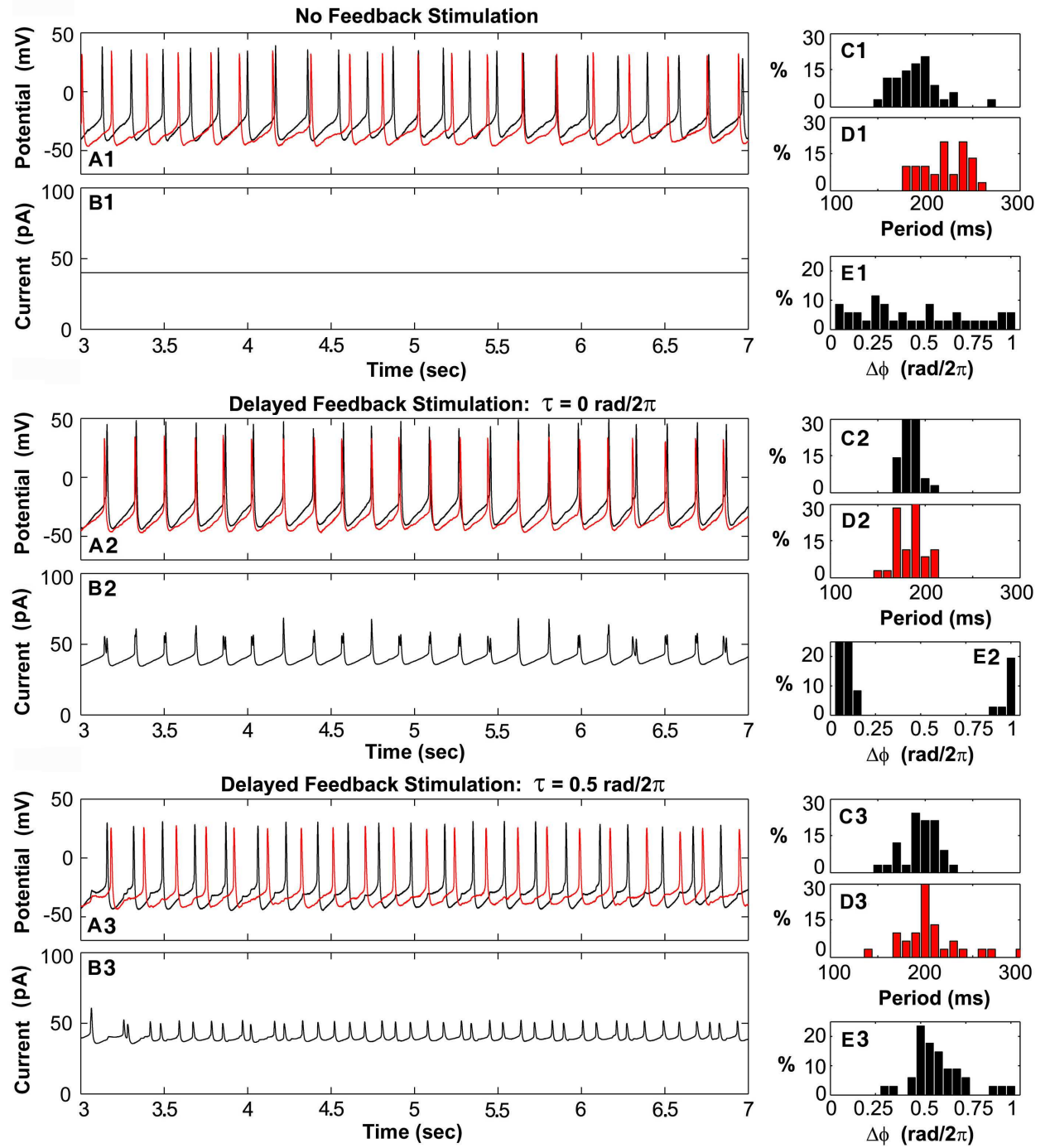

Figure 4.4: Experimental results for two patch clamped neurons under linear global feedback. A) Membrane potential recording of 2 neurons. B) Applied stimulation current $\left(K_{o}=13 \mathrm{mV}, K=400\right)$. C \& D) Period distribution of neuron action potentials of neuron $1 \& 2$ respectively. E) Observed distribution of phase differences between the action potentials of the two neurons. 
delay of $\tau=0 \mathrm{rad} / 2 \pi$ caused the mean period of the two cells to lock with a period of approximately $180 \mathrm{~ms}$ (Figure $4.4 \mathrm{C} 2 \& \mathrm{D} 2$ ). The mean phase difference between the neuron action potentials was observed to be approximately $0.05 \mathrm{rad} / 2 \pi$, indicating the presence of a nearly in-phase synchronized state (Figure 4.4 E2). This observation was consistent with theoretical expectations (Figure $4.3 \mathrm{C}$ ). The in-phase synchronized state persisted until the feedback stimulation was removed (not shown). Increasing the feedback delay to $\tau=0.5 \mathrm{rad} / 2 \pi$ caused the action potentials of the two neurons to synchronize in an anti-phase configuration (Figure 4.4 A3-E3). This was consistent with expectations (Figure 4.3 D).

\subsubsection{Predictions for a Large Population of Neurons}

Models constructed from experimental measurements on single neurons have proved effective for estimating stimulation parameters for controlling the action potential synchronization of two neurons. However, the ultimate goal is the creation (or disruption) of synchronization in large-scale systems. To demonstrate this

application, the experimentally constructed phase model was utilized to estimate stimulation parameters which would disrupt the collective synchronization of a large population of globally coupled neurons. These parameters can be used as a starting point to design a feedback signal that would effectively shorten the duration and severity of seizures in animal models. The dynamical behavior of such a system is governed by the superposition of all interactions (internal and external) between elements.

$$
\frac{d \phi_{i}}{d t}=\omega_{i}+\sum_{j=1}^{N} H_{\text {int }}\left(\phi_{j}-\phi_{i}\right)+H_{\text {ext }}\left(\phi_{j}-\phi_{i}\right) \quad \text { for } i=1 \ldots N
$$


Table 4.1: Analytical expressions for the real parts of the eigenvalues of the first 4 balanced cluster states, assuming the highest order harmonic in the interaction function is $\leq 6$ (from Rusin [115]).

\begin{tabular}{ccc}
\hline Cluster State & $\lambda_{1}$ & $\lambda_{2}$ \\
\hline 1 & $-A_{1}-2 A_{2}-3 A_{3}-4 A_{4}-5 A_{5}-6 A_{6}$ & - \\
2 & $A_{1}-2 A_{2}+3 A_{3}-4 A_{4}+5 A_{5}-6 A_{6}$ & $-2 A_{2}-4 A_{4}-6 A_{6}$ \\
3 & $1 / 2 A_{1}+A_{2}-3 A_{3}+2 A_{4}+5 / 2 A_{5}-6 A_{6}$ & $1 / 2 A_{1}+A_{2}-3 A_{3}+2 A_{4}+5 / 2 A_{5}-6 A_{6}$ \\
4 & $1 / 2 A_{1}+3 / 2 A_{3}-4 A_{4}+5 / 2 A_{5}$ & $2 A_{2}-4 A_{4}+6 A_{6}$ \\
\hline \hline Cluster State & $\lambda_{3}$ & $\lambda_{4}$ \\
\hline 1 & - & - \\
2 & - & - \\
3 & $-3 A_{3}-6 A_{6}$ & $-4 A_{4}$ \\
\hline
\end{tabular}

Typically the intrinsic interactions $\left(H_{\text {int }}\right)$ of such a system are unknown. The application of an external stimulation is utilized to overwhelm the unknown intrinsic coupling between elements allowing new dynamical behaviors to be artificially created. The challenge is in picking the stimulation parameters which will produce the desired effect on the collective behavior of the target system.

To create a desynchronized state, all stationary states of the system must be simultaneously destabilized. The stability of synchronized states can be determined by calculating the eigenvalues associated with these states. Assuming that only balanced phase cluster states are possible, the associated eigenvalues can be determined from the Fourier coefficients of the net interaction function (see Equation $4.8)[121,122]$. Equations for cluster states $1-4$ with up to $6^{\text {th }}$ order harmonics are listed in Table 4.1. To actively disrupt phase synchronized states in a rhythmic population, a set of feedback parameters must be selected such that all phase cluster states have at least 1 eigenvalue with positive real part.

Having experimentally obtained the response function of the experimental neuron system, the stability of balanced phase cluster states can be determined as a 
function of the stimulation parameters. Only linear feedback will be considered for this analysis. By adjusting the feedback delay parameter, the stability of the synchronized states can be externally influenced; the amplitude of the feedback signal does not effect the stability of such states. The eigenvalues of cluster states 1-4 were calculated as a function of feedback delay for the experimental neuron system under first order feedback (Figure 4.5). The eigenvalues indicate that the external feedback signal will desynchronize a population of globally coupled neurons when the feedback delay is between $0.35-0.45 \mathrm{rad} / 2 \pi$. In this parameter range each cluster state will have at least 1 eignevalue with a positive real part. This parameter range is believed to be large enough to provide a robust starting point for future experiments.

\subsection{Discussion}

Current research has explored the synchronization behavior of neurons as a function of inhibitory and excitatory synaptic connections [123-125]. Here we demonstrate that the phase model can also be used to generate global electrical stimulation parameters which can potentially overcome natural behaviors of a neural system.

Phase based models have proven to be a valuable tool for characterizing the individual and collective dynamical behavior of neurons [97, 98, 102, 106, 107]. Such models are advantageous since no detailed knowledge of the biochemistry of neurons is required; only macroscopic measurements of membrane potential are necessary for model construction. Typically, models are generated from experimental measurements on a single neuron and subsequently used to infer the synchronization behavior of a population of interacting neurons. We reverse the process by using the phase model to determine the stimulation required to produce 

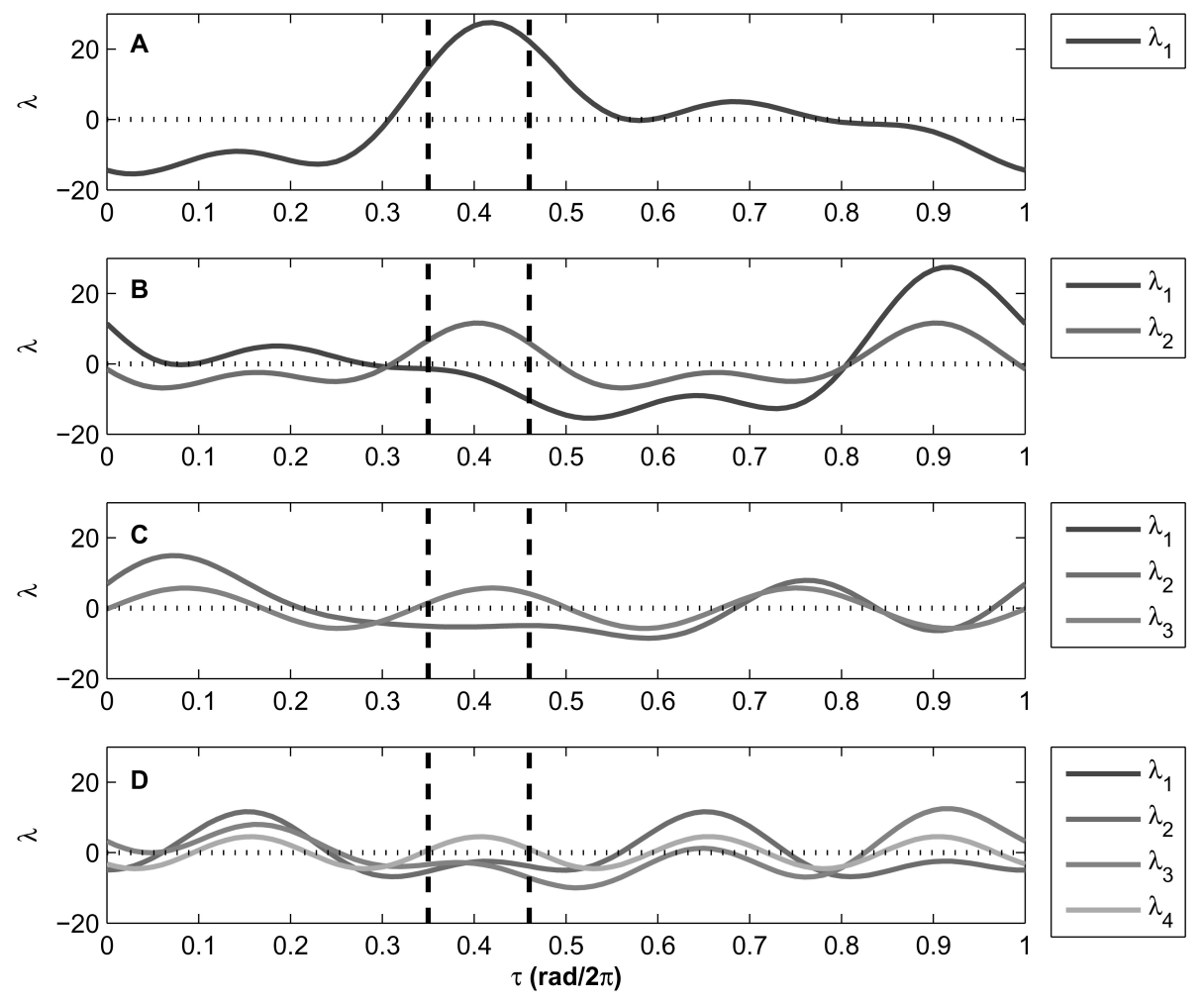

Figure 4.5: Eigenvalues calculated for balanced cluster states as a function of feedback stimulation delay. A) 1-Cluster state. B) 2-Cluster state. C) 3-Cluster state. D) 4-Cluster state. Dashed lines indicate region of possible desynchronization. In the 3 -Cluster state $\lambda_{1}=\lambda_{2}$, and in the 4-Cluster state $\lambda_{1}=\lambda_{3}$ (see Table 4.1).

a desired synchronization behavior.

The use of model derived feedback signals for controlling the synchronization behavior of neurons may represent an improvement over the ad-hoc methods of parameter estimation for current neurostimulation therapies. We have previously demonstrated that phase models can be used to engineer global feedback stimulations for controlling the collective behavior of large populations of complex rhythmic elements in nonlinear electrochemical systems [90]. This work demonstrates proof of concept for applying our engineering framework to control the synchronization behavior of biological neurons. As seen in Figure 4.4, both in-phase 
and anti-phase configurations were successfully generated using global delayed feedback. In both cases, a single common stimulation signal was applied equally to the neurons. Such global stimulation is required for clinical neurostimulation applications. Previous work has demonstrated the use of addressable electrical stimulation for influencing the synchronization of neurons [126].

The effect of synaptic connections on the synchronization of neurons was not considered due to experimental limitations. However, past work has shown that synaptic connectivity and interactions may be directly incorporated into the phase model $[125,127]$. Previous work has also demonstrated that stimulation signals can be created to overwhelm intrinsic interactions between elements and guide the target system towards the desired state [90]. The use of nonlinear stimulation for controlling populations of neurons has been previously demonstrated in numerical simulations [128].

The use of a phase model for estimating neurostimulation parameters is subjected to limitations. As derived, the phase model requires the use of relatively periodic elements. Since neurons under physiological conditions have long quiescent periods, describing their natural behavior by such model may not be possible. However, neurological events such as seizures have long episodes of roughly periodic neuronal activation which may be described using such models [129].

Additionally, the use of phase models requires that the stimulation signal remains small such that the amplitude of the action potential remains undisturbed. However, weak feedback stimulation is desirable since it minimizes disruption of the natural rhythmic behavior of the neurons. This can be seen in Figure 4.2, where upon the removal of the feedback stimulation, the period distribution of the action potentials of the neurons return to their pre-stimulation baseline distribution. No permanent changes to the neuron were observed. 


\subsection{Conclusions}

A method of constructing a phase model, using time delayed self-feedback, has been developed; this method requires experimental access to only a single representative rhythmic unit. After constructing the phase model from observations of the action potential of a single neuron, it was used to predict the synchronization states of a two neuron system. Experiments were conducted to observe the phase behavior of the action potentials of two neurons under linear time-delayed feedback. The predictions of the phase model were confirmed by the experimental observations. This method provides an additional approach for the construction of dynamical models of complex rhythmic systems. 


\section{Chapter 5}

\section{Synchronization of Low Magnesium Bursting In Vitro ${ }^{1}$}

\subsection{Introduction}

In this chapter, the relationship between synchronization and synaptic strength is explored. Synchronization of action potentials for dual whole cell recordings in various $\mathrm{Mg}^{2+}$ external conditions were calculated. The change in synaptic strength were analyzed using two methods: the method of failures and histograms of excitatory postsynaptic current (EPSC) amplitudes. Finally, the effect of three conventional antiepilpetic drugs (AEDs) with different mechanisms on action potential synchronization was examined.

\footnotetext{
${ }^{1}$ S.E. Johnson, J.L. Hudson and J. Kapur, "Synchronization of Action Potentials During Low Magnesium Bursting", (in preparation).
} 


\subsection{Background}

Seizures are thought to be the result of hypersynchronous neural activity [3]. Current research is trying to address the synchronization properties of action potentials to improve treatment of epilepsy patients $[30,99,124,130]$. Synchronization of bursting activity is a characteristic of many in vitro epilepsy models [28, 31, 34, 36$38,130-132$ ] even though the underlying mechanisms causing synchronized bursting may be different [27]. Often the synchronization of the bursting activity is examined using cross-correlation [28-30], field potentials [31-33] or by visual inspection [34-38]. Few experimental studies quantify the synchronization of action potentials to make comparisons between various in vitro conditions.

It is possible for action potential synchrony to exist in neurons that have synaptic connections between them $[89,125,133]$. An underlying assumption of in vitro seizure activity is that the neural activity is more synchronized during a seizure than under more 'natural' external conditions where no bursting is present. Under bursting conditions, action potentials in connected cells do not always exhibit exact synchronization [130], but there may be varying time delays [38] for which action potentials could be considered synchronous.

The strength of synaptic connections can be determined using quantal analysis. Each vesicle in the presynaptic neuron has "unit" of neurotransmitters called a quanta [134]. The unit of neurotransmitters causes a specific amplitude response in the postsynaptic neuron upon release. Measurements of single quanta release can be done under conditions of low probability of release, such as high $\mathrm{Mg}^{2+}$ and low $\mathrm{Ca}^{2+}$, or no action potential firing with tetrodotoxin (TTX). Responses under these conditions are mini-excitatory postsynaptic currents or potentials (mEPSCs, mEPSPs). Recordings with action potentials present in physiologically similar 
concentrations of $\mathrm{Mg}^{2+}$ and $\mathrm{Ca}^{2+}$ are called spontaneous excitatory postsynaptic currents or potentials (sEPSCs, sEPSPs). The sEPSPs (or sEPSCs) will occur with amplitudes that are multiples of the mEPSP (or mEPSCs) [134]. The mean quantal content, $m$, is the mean number of quanta released after a presynaptic action potential. The mean quantal content increases as the sEPSC (or sEPSP) amplitudes increase and as failures decrease; both indicate that synaptic strength has increased.

Low and Zero $\mathrm{Mg}^{2+}$ in the extracellular solution can be used as an seizure-like condition [27, 34]. Decreasing $\mathrm{Mg}^{2+}$ creates bursting behavior predominantly by unblocking NMDA receptors $[34,135]$. Bursts in decreased $\mathrm{Mg}^{2+}$ conditions were shown to be synchronized using extracellular measurements [132]. Additionally, decreasing $\mathrm{Mg}^{2+}$ has been shown to increase the amplitude and frequency of sEPSCs [135]. This suggests that the strength of the synaptic connections has increased. Mangan and Kapur [135] implied that the increase in synchronized activity is related to the increase in synaptic strength, but the relationship between synaptic strength and synchronization has not been studied directly.

\subsection{Methods}

\subsubsection{Mixed Culture Preparation}

Animals were treated according to a protocol approved by the University of Virginia Health Sciences Center Animal Research Committee and efforts were made to minimize animal stress and discomfort. Cultures were prepared from P0-P1 Sprague-Dawley newborn rats using methods similar to those described elsewhere $[116,117]$. The newborn rats were decapitated, their brains removed and placed in cold HEPES-buffered Hank's balanced salt solution (HEPES-HBSS). The hippocampi were removed under a dissecting microscope and collected in a small 
petri dish containing HEPES-HBSS. Tissues were incubated in $0.125 \%$ trypsin for $15 \mathrm{~min}$ at $37^{\circ} \mathrm{C}$. Trypsin solution was replaced with $5 \mathrm{~mL}$ HEPES-HBSS and the cells were rinsed twice more with HEPES-HBSS at 5 min intervals. Hippocampi were triturated until no fragments of tissue remained. Neurons were collected by centrifugation and resuspended in $5 \mathrm{~mL}$ of Dulbeccos modified Eagles medium (DMEM) and F-12 supplement (1:1) (Invitrogen) with $10 \%$ fetal bovine serum (heat-inactivated, Invitrogen), 2 mM L-glutamine (Invitrogen), and penicillin (100 $\mathrm{U} / \mathrm{mL})$-streptomycin $(100 \mathrm{U} / \mathrm{mL})$. Culture dishes were coated with poly-lysine and filled with $2 \mathrm{~mL}$ of culture medium. Cells were plated at a minimum density of 50,000 per $35 \mathrm{~mm}^{2}$ dish and kept at $37^{\circ} \mathrm{C}$ in a $5 \% \mathrm{CO}_{2}$ incubator. After $24 \mathrm{~h}$, the culture medium was changed to serum-free medium containing $2 \%$ B27 and 2 $\mathrm{mmol} / \mathrm{L}$ glutamine. The medium was replaced with fresh medium every 2-3 days.

\subsubsection{Electrophysiology}

\section{Experimental Setup}

Neurons were 14 days in vitro (DIV) at time of use. Three external media were used containing various concentrations of $\mathrm{Mg}^{2+}$ : Control Medium (CM) containing 3 $\mathrm{mM} \mathrm{Mg}^{2+}$, Low Magnesium (LM) containing $0.5 \mathrm{mM} \mathrm{Mg}^{2+}$, and Zero Magnesium (ZM) with $0 \mathrm{mM}$ added $\mathrm{Mg}^{2+}$. The external media consisted of either (in $\mathrm{mM}$ ): 147 $\mathrm{NaCl}, 2.6 \mathrm{KCl}, 2 \mathrm{CaCl}_{2}, 3 \mathrm{MgCl}_{2}, 10$ glucose, and 10 HEPES, pH 7.4, osmolarity 310-316 mOsm (CM); $153 \mathrm{NaCl}, 2.6 \mathrm{KCl}, 2.1 \mathrm{CaCl}_{2}, 0.5 \mathrm{MgCl}_{2}, 10$ glucose, and 10 HEPES, pH 7.4, osmolarity 314-316 mOsm (LM); $153 \mathrm{NaCl}, 2.6 \mathrm{KCl}, 2.2 \mathrm{CaCl}_{2}, 10$ glucose, and 10 HEPES, pH 7.4, osmolarity 314-316 mOsm (ZM). Depending on the experiment, $40 \mu \mathrm{M}$ of 5,5-diphenylhydantoin sodium salt (PHT), 50-100 $\mu \mathrm{M}$ levetiracetam (LEV) or $75 \mu \mathrm{M}$ GYKI-52466 (GYKI) was added to the ZM external 
solution from stock solutions of PHT (in $0.2 \mathrm{M} \mathrm{NaOH}$ ), LEV (in ZM) and GYKI (in DMSO). The final concentration of DMSO and $\mathrm{NaOH}$ were less than $0.3 \%$. The external medium was sterile filtered before use. In solution-switch experiments and AED experiments with PHT, LEV or GYKI, a perfusion system was used to exchange the medium while maintaining the dual patch.

Micropipettes were pulled on a P-97 Flaming/Brown Micropipette puller (Sutter Instruments, Novato, CA) from borosilicate glass (O.D. $1.5 \mathrm{~mm}$, I.D. $0.86 \mathrm{~mm}$, World Precision Instruments, Sarasota, FL) using a 1-stage pull protocol. Micropipettes with resistances of 5-10 M $\Omega$ s were filled with a solution of (in $\mathrm{mM}$ ): $155 \mathrm{~K}$-gluconate, 0.6 EGTA, 12 HEPES, $8 \mathrm{KCl}, 3 \mathrm{NaCl}$ and $4 \mathrm{MgATP}, \mathrm{pH}$ 7.3, osmolarity 297-301 mOsm. The internal solution was sterile filtered before use. In the voltage-clamp recordings, lidocaine $\mathrm{N}$-ethyl bromide was added to the internal solution to block action potentials. HEPES, $\mathrm{K}$-gluconate, $\mathrm{KOH}, \mathrm{KCl}, \mathrm{MgCl}_{2}$, lidocaine, glucose, PHT, MgATP, EGTA, LEV and DMSO were from Sigma-Aldrich (St. Louis, $\mathrm{MO}$ ); $\mathrm{NaCl}$, $\mathrm{CaCl}_{2}$ and $\mathrm{NaOH}$ were from Fischer Scientific (Pittsburg, PA); GYKI was from TOCRIS bioscience (Ellisville, MO).

The apparatus described in Section 4.4.3 was modified to record from two neurons in the same dish. A schematic of the dynamic clamp system can be seen in Figure 5.1. A separate silver chloride reference/counter electrode was placed in the dish for each cell. Experiments were done at room temperature and cultured neurons were viewed on a Scientifica stage (Uckfield, East Sussex, UK) using an inverted Nikon (Melville, NY) microscope. Distance between cells was measured using LinLab (Uckfield, East Sussex, UK). All dual patched cells were within $450 \mu \mathrm{m}$ of one another. Micropipettes were mounted on SD Instruments micro-manipulators (Grants Pass, OR). Data were recorded at $25 \mathrm{kHz}$ using an FPGA card and user defined LabVIEW software (National Instruments Corporation, Austin, TX) with an 


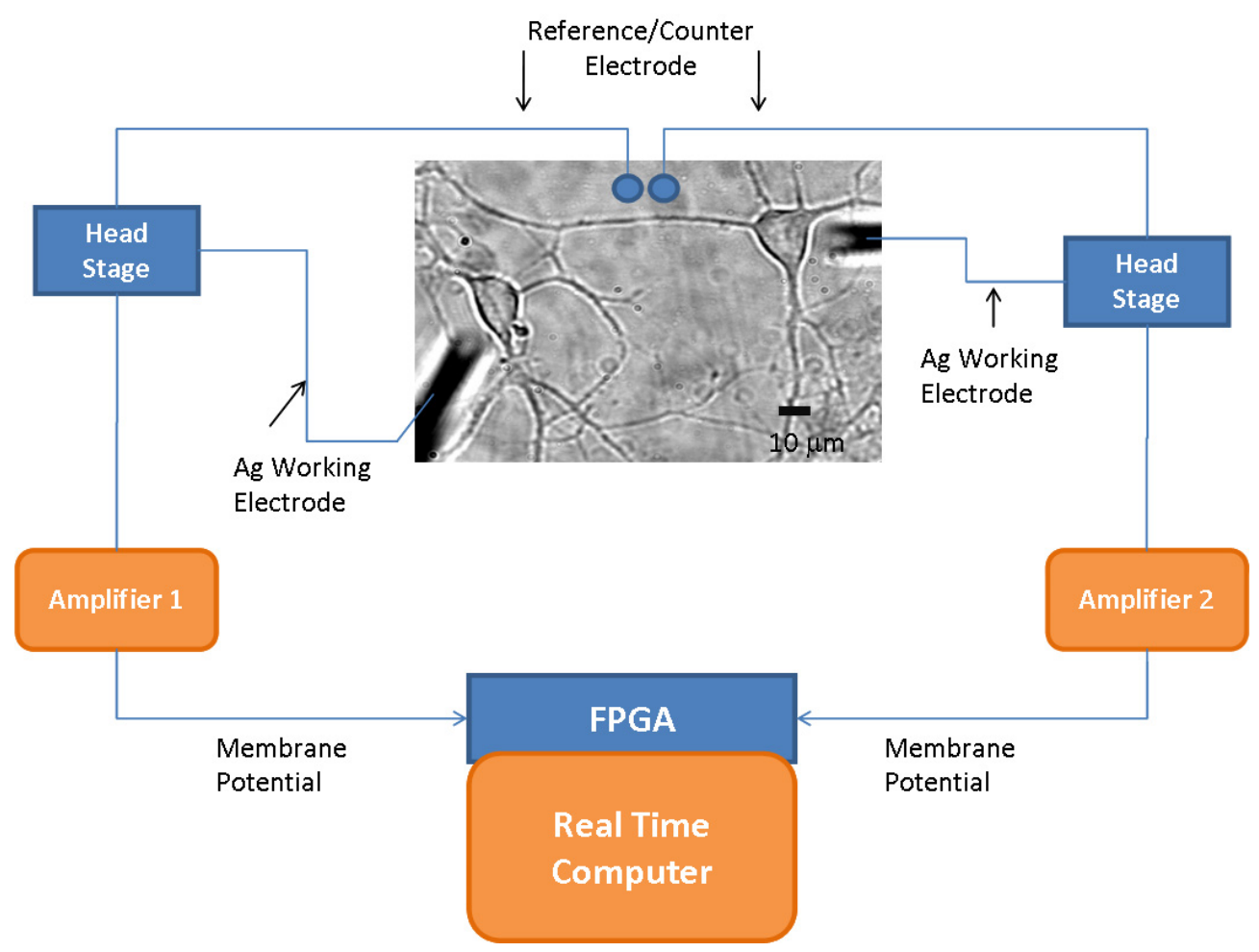

Figure 5.1: Schematic of the dual patch clamp apparatus.

Axopatch 200A and an Axopatch 200B amplifiers (Molecular Devices, Sunnyvale, CA), filtered using an 4-pole Bessel filter at $5 \mathrm{kHz}$. The adjustable analog output scaling factor, $\alpha$, was set to 50 for the dual current-clamp recordings and 10 for the simultaneous current-clamp and voltage-clamp recordings. Only the amplifiers were used to apply current or potential.

\section{Dual Current-Clamp Recordings}

Connections were explored in CM. Dual patch-clamped cells were recorded in current-clamp mode. One cell was given a suprathreshold current (presynaptic cell) and the other cell was hyperpolarized to $\sim-65 \mathrm{mV}$ (postsynaptic cell). Then the process was repeated by switching the presynaptic and postsynaptic cell.

In $\mathrm{CM}$, both cells were depolarized with a suprathreshold current to generate 
action potentials in both cells. In ZM and LM, cells were either depolarized with a supratheshold current, or if bursting was present the cells were injected with the appropriate current to set the minimum membrane potential to $\sim-65 \mathrm{mV}$.

In grouped experiments, cells were measured in either $\mathrm{CM}$ or $\mathrm{ZM}$ conditions only. In solution-switched experiments, cells were initially patch-clamped in CM and then the solution was exchanged using a whole bath perfusion system to either LM or ZM conditions. In experiments with AEDs, the cells were initially patchclamped in $\mathrm{CM}$, the solution was exchanged to $\mathrm{ZM}$ and then again exchanged to $\mathrm{ZM}+\mathrm{AED}$. If no bursting was present in $\mathrm{ZM}$ at $\sim-65 \mathrm{mV}$ for cells used in the AED experiments, the cells were discarded.

\section{Simultaneous Current-Clamp and Voltage-Clamp Recordings}

Whole cell recordings of two neurons were made in $\mathrm{CM}$, then the solution was switched to LM and then to ZM. The presynaptic neuron was measured in currentclamp mode and the postsynaptic neuron was measured in voltage-clamp mode. The external solution was exchanged using a bath perfusion system. The presynaptic neuron was injected with a suprathreshold current and the postsynaptic neuron was held at a potential of $-65 \mathrm{mV}$. Postsynaptic neurons that required $<-150 \mathrm{pA}$ to hold the potential in CM were discarded.

\subsubsection{Data Analysis}

\section{Event Synchronization}

The event synchronization (ES) described by Quiroga et al. [136] was used to quantify the degree of action potential synchronization. ES quantifies the number

of times an event occurs in both time series within a small window. This measure 
can tell whether the two time series have events that are consistently together and if one time series leads the other. A brief description of the ES is below, but more details are available elsewhere [136].

For two neurons, action potential times in neuron $x$ and $y$ can be defined as $t_{i}^{x}$ and $t_{j}^{y}$. Action potentials that occur in both neurons within a time interval, $\pm \tau$, are considered to be synchronous. For each neuron a count of these events is defined as

$$
c^{\tau}(x \mid y)=\sum_{i=1}^{n_{x}} \sum_{j=1}^{n_{y}} J_{i j}^{\tau}
$$

with

$$
J_{i j}^{\tau}= \begin{cases}1, & \text { if } 0<t_{i}^{x}-t_{j}^{y} \leq \tau \\ 1 / 2, & \text { if } t_{i}^{x}=t_{j}^{y} \\ 0, & \text { else }\end{cases}
$$

where $n_{x}$ is the number of action potentials in neuron $x$, and $n_{y}$ is the number of action potentials for neuron $y$, with $i=1, \ldots, n_{x}$ and $j=1, \ldots, n_{y}$. The values of $c^{\tau}(x \mid y)$ and $c^{\tau}(y \mid x)$ are then combined symmetrically $(Q)$ and antisymmetrically $(q)$ :

$$
\begin{aligned}
& Q_{\tau}=\frac{c^{\tau}(y \mid x)+c^{\tau}(x \mid y)}{\sqrt{n_{x} n_{y}}} \\
& q_{\tau}=\frac{c^{\tau}(y \mid x)-c^{\tau}(x \mid y)}{\sqrt{n_{x} n_{y}}}
\end{aligned}
$$

where $Q_{\tau}$ is the strength of the ES and $q_{\tau}$ demonstrates the bias of the events. $Q_{\tau}$ varies from 0 to 1 with 0 being no synchronized events and 1 being completely synchronized events. $q_{\tau}$ can vary from -1 to 1 , where -1 means that the events in neuron $y$ always occur before the events in neuron $x$, and 1 is the opposite order. A $q_{\tau}$ value of zero would mean that the neurons showed no preferred order for the 
synchronized events.

To avoid double counting, a local definition of $\tau$ for each event pair $i j$ is defined as:

$$
\tau=\min \left\{\tau_{c}, \frac{t_{i+1}^{x}-t_{i}^{x}}{2}, \frac{t_{i}^{x}-t_{i-1}^{x}}{2}, \frac{t_{j+1}^{y}-t_{j}^{y}}{2}, \frac{t_{j}^{y}-t_{j-1}^{y}}{2}\right\} .
$$

where $\tau_{c}$ is a constant representing the maximum allowable time interval between action potentials.

\section{Quantal Analysis}

Quantal analysis was done using two methods, the method of failures and amplitude histogram analysis [134]. Failures were identified in windows of $30 \mathrm{~ms}$ following an action potential in the presynaptic neuron. First, the excitatory postsynaptic current (EPSC) onsets were determined by 5 consecutive negative derivatives in the current of the postsynaptic neuron [137]. Then, the falling phase of the EPSC was determined by the sum of the derivative being over $0.0156 \mathrm{pA} / \mathrm{ms}$ over a moving window of $1 \mathrm{~ms}$ [137]. If the minimum value between the onset and falling phase was greater than 3 standard deviations of the RMS noise it was considered a 'success.' If there was no onset or if the minimum was not greater than 3 standard deviations of the noise, the response was considered a 'failure.' The number of failures, $N_{0}$, and number of action potentials, $N$, were combined to create the mean quantal content, $m_{f}=-\log \left(N_{0} / N\right)$. For cells where no failures were measured, a value of $N_{0}=1$ was used to obtain a $m_{f}$ for comparison purposes.

The method of amplitude histogram analysis was done on all EPSC amplitudes in the first two minutes of the recording of the postsynaptic neuron. EPSC amplitudes were analyzed using MiniAnalysis (Synaptosoft, Decantur, GA). The threshold was set to three times the root mean square of baseline noise. Amplitudes were 
discarded if they met either of the following sets of criteria: 1) the decay time was greater than the rise time, the amplitude was less than $40 \mathrm{pA}$ and the peak area was less than 150 pAms or 2) the decay time was greater than $10 \mathrm{~ms}$, the rise time was greater than $5 \mathrm{~ms}$ and the amplitude was less than $40 \mathrm{pA}$. Amplitude histograms were fit with Gaussian curves using a MATLAB (Natick, MA) program created by T. C. O’Haver.

\section{Classification of Synaptic Connections}

Responses in the postsynaptic cell as a result of action potentials in the presynaptic cell were analyzed using a user defined program in MATLAB. First, action potentials $>100 \mathrm{~ms}$ apart were identified in the presynaptic cell. The $100 \mathrm{~ms}$ limit avoids convolution of responses from multiple action potentials. Then the mean (overall baseline) and standard deviation (overall noise) of the membrane potential in postsynaptic cell was measured. For each action potential in the presynaptic cell, a minimum and maximum of the membrane potential of the postsynaptic cell were found within a window of $30 \mathrm{~ms}$ from the peak of the action potential. Maximum and minimum values at the edge of the $30 \mathrm{~ms}$ window were rejected, and then program moved to the next action potential. The mean and standard deviation of a $3 \mathrm{~ms}$ window just prior to the peak of the action potential created the local baseline and local noise, respectively. If the local baseline is greater than the overall baseline $\pm 2 \mathrm{mV}$, or the local noise was greater than the 1.25 times the overall noise or a maximum of $1 \mathrm{mV}$, the response was rejected and the program moves to the next action potential. The membrane potential was averaged for $3 \mathrm{~ms}$ at the minimum and maximum values and the local baseline was subtracted to get the maximum amplitude and minimum amplitude within the $30 \mathrm{~ms}$ window. If the minimum amplitude was greater than three times the overall noise, the response 
was identified as inhibitory. If the maximum amplitude was greater than three times the overall noise, the response was identified as excitatory. Otherwise the response was considered a failure. For excitatory responses, the time from the peak of the presynaptic action potential to the peak of the excitatory postsynaptic potential was recorded.

Connections were classified as one of four categories: strong excitatory (s), weak excitatory (w), inhibitory (i) and electrical (e). Strong exciatatory connections were those that had average excitatory responses greater than $30 \mathrm{pA}$ or $3 \mathrm{mV}$ with less than $10 \%$ failures. If the response had a value lower than three times the RMS noise, it was classified as inhibitory. Electrical responses were determined by visual inspection. All other responses, including all failures, were considered weak excitatory connections.

\section{Burst Analysis}

Identification of bursts was done using a user defined program in MATLAB similar to the methods by Pasquale et al. [138], Selinger et al. [139]. First, the action potentials were identified and the inter-spike intervals (time between subsequent spikes, ISI) were calculated. A logISI histogram was created using equally spaced bins of 0.1 in $\log _{10}$ (ISI) units $[138,139]$. Next, the histrogram was smoothed using weighted linear least squares and a 1st degree polynomial model in MATLAB (smooth with lowess method). Peaks of the smoothed histogram were identified in MATLAB using findpeaks with a minimum difference of 2 between peaks. The minimum between the two peaks was identified as the burst threshold. If only one peak was found, the data were identified as having no busrts. If there were more than two peaks, then peaks greater than 10 seconds were ignored. If two peaks were less than 10 seconds, the minimum between those two peaks was identified 
as the burst threshold. If there were less than 1 peaks below 10 seconds, then the minimum between the two smallest (logISIs) was the burst threshold. Finally, if more than two peaks were below 10 seconds, then the minimum between the two largest (logISIs) peaks under 10 seconds was defined as the burst threshold. ISIs longer than the burst threshold were used to identify the beginning and end of bursts and each burst had to contain $\geq 3$ action potentials [140].

\subsubsection{Statistics}

Significance was determined by $\alpha<0.05$ for all statistical tests performed. Data were reported as means \pm SD unless noted otherwise. Statistical tests used were Student's t-test (MATLAB), ANOVA (MATLAB), repeated measures ANOVA (rmANOVA, Minitab 16, State Collage, PA), Fisher's Exact Test (Fishers, MATLAB, program created by Giuseppe Cardillo) and Kolmogorov-Smirnov test (K-S test, MATLAB) as noted in the text and figures. Post-hoc multicomparison tests were done using the Tukey method (MATLAB, Minitab). Number of pairs are denoted as $n_{p}$ and number of individual cells are denoted as $n_{c}$ through out the chapter.

\subsection{Results}

\subsubsection{Neuronal Properties}

\section{Summary of Pairs Recorded in Current-Clamp Mode}

A total of 20 pairs of neurons were examined for the grouped experiments. Of the 20 pairs, 13 were recorded in control medium (CM) only. The other 7 pairs were recorded from in zero added $\mathrm{Mg}^{2+}(\mathrm{ZM})$. In solution-switched experiments, 17 pairs were measured in $\mathrm{CM}$ and switched to $\mathrm{ZM}$. Another 9 pairs were measured in $\mathrm{CM}$ 
and switched to low $\mathrm{Mg}^{2+}$ (LM) solutions. To examine the effect of antiepileptic drugs (AEDs) on synchronization, 4 pairs of neurons were recorded for each AED. The pairs of neurons were recorded in $\mathrm{CM}$, switched to $\mathrm{ZM}$ and finally switched to $\mathrm{ZM}+\mathrm{AED}$. Behavior of the individual cells in CM, LM and ZM are described below. Results for the pairs of cells can be found in their respective sections.

\section{Control Medium}

Control medium (CM) contained $3 \mathrm{mM} \mathrm{Mg}^{2+}$ in the external solution. Cell pairs where one or both cells did not fire action potentials when subjected to a suprathreshold current were discarded $\left(n_{p}=5\right)$. Rarely, neurons in CM fired spontaneous action potentials when held near a membrane potential of $-65 \mathrm{mV}\left(n_{p}=2 / 72\right)$. Occasionally, bursting activity was seen in $\mathrm{CM}\left(n_{p}=4\right)$ at potentials of $-65 \mathrm{mV}$. The bursting activity was not continuous, and these pairs were either discarded $\left(n_{p}=3\right)$ or sections of data without bursting activity were used for further analysis $\left(n_{p}=1\right)$.

When a suprathreshold current was injected to cells in CM, three types of activity were commonly seen: recurrent action potentials $\left(n_{c}=73\right)$, bursts of action potentials $\left(n_{c}=17\right)$ or action potentials only at the beginning of the depolarization $\left(n_{c}=11\right.$, Figure 5.2).

Decreased extracellular $\mathrm{Mg}^{2+}$ has been shown to cause recurrent bursting activity $[28,34,130,132]$. Two decreased $\mathrm{Mg}^{2+}$ models were used, containing $0.5 \mathrm{mM}$ $\mathrm{Mg}^{2+}$ (low magnesium, $\mathrm{LM}$ ) or $0 \mathrm{mM} \mathrm{Mg}^{2+}$ (zero added magnesium, $\mathrm{ZM}$ ). In both conditions, spontaneous bursting activity was observed at potentials of $\sim-65 \mathrm{mV}$. Nine pairs of neurons in LM or ZM were depolarized to generate action potentials when bursting was not present (mean minimum potential, $-52.1 \pm 0.5 \mathrm{mV}, n_{c}=18$ ). They were not included in the bursting analysis. The burst duration, number of action potentials per burst and burst threshold were identified using a user defined 


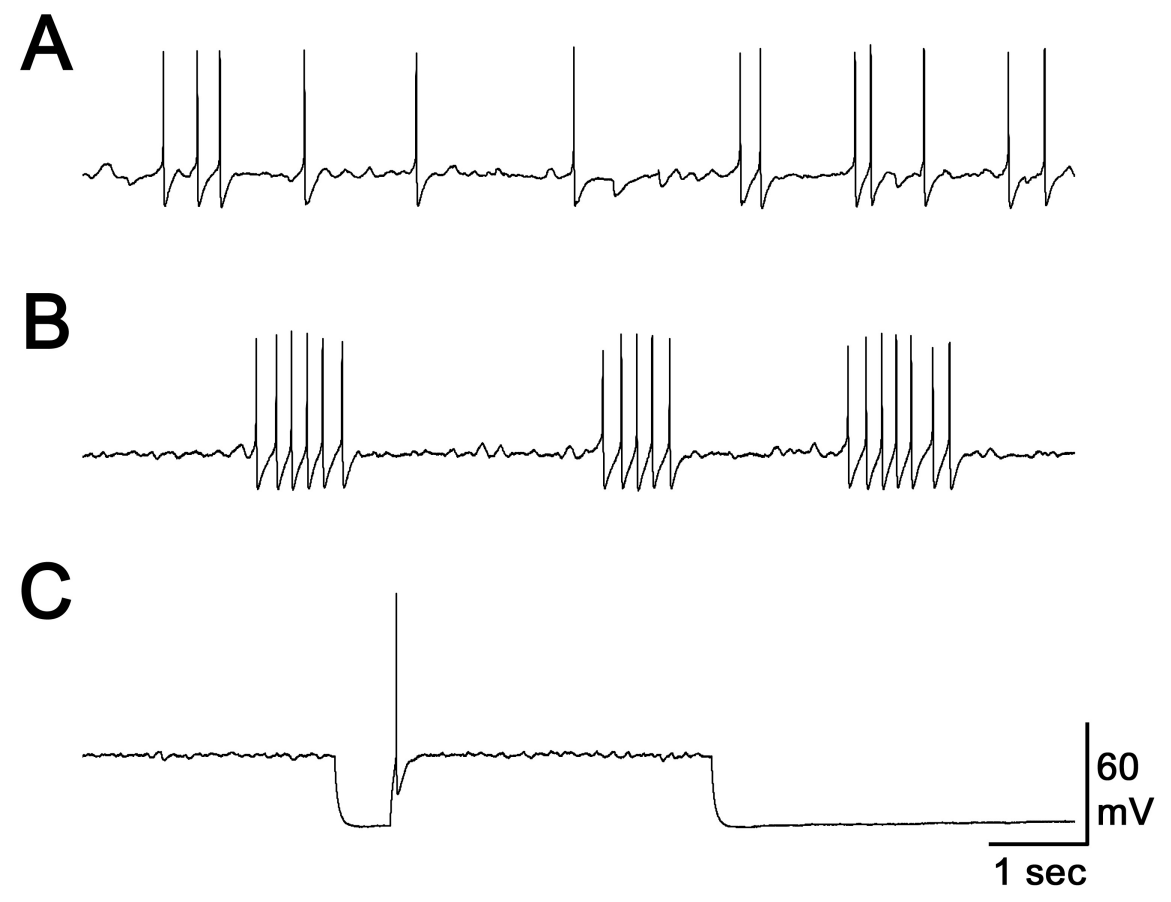

Figure 5.2: Whole cell recordings in current-clamp mode for cells in CM. A) Trace of a cell with recurrent action potential. Cells could also have more periodic action potentials as well. B) Trace for a cell with bursts of action potentials. C) Trace for a cell that only fired action potentials with application of a depolarizing current. Application of suprathreshold current in $\mathrm{A}$ and $\mathrm{B}$ was constant and lasted the duration of the recording. In $\mathrm{C}$ the suprathreshold current was applied and released as evident by the sharp change in membrane potential during the trace.

program. The burst threshold varied depending on the $\log _{10}$ interspike interval (ISI) histogram for each cell. The logISI histograms had either 1 peak (no bursts, Figure 5.3 A and B), two peaks (Figure 5.3 C and D) or three peaks (Figure 5.3 $\mathrm{E}$ and F). The burst threshold was identified as the local minimum between the two peaks of the logISI histograms as described in Section 5.3.3 (Figure 5.3 C and E, dashed line). Burst thresholds for LM cells were 1.07 $\pm 1.64 \mathrm{sec}\left(n_{c}=23\right)$. Burst thresholds for ZM cells were $0.57 \pm 0.47 \sec \left(n_{c}=32\right)$. The burst thresholds were not significantly different $(\mathrm{p}>0.1$, $\mathrm{t}$-test). The number of action potentials per burst for 
LM was $17.6 \pm 32.5\left(n_{c}=23\right)$ and ZM was $10.2 \pm 16.7\left(n_{c}=32\right)$, which was significantly different $(\mathrm{p}<0.0001, \mathrm{t}$-test $)$. Burst durations were $2.46 \pm 4.70 \mathrm{sec}$ in $\mathrm{LM}\left(n_{c}=23\right)$ and $1.50 \pm 2.66 \mathrm{sec}$ in $\mathrm{ZM}\left(n_{c}=32\right)$, which were significantly different $(\mathrm{p}<0.0001, \mathrm{t}$-test $)$. From these data, it can be concluded that bursts in LM are longer and include more action potentials than bursts in ZM. However the standard deviations for these measures are large and no particular bursting activity was consistently seen in either condition. This suggests that the bursting duration and number of action potentials were most likely a property of the specific culture (number of cells, density of connections, type of connections), not an inherent property of the external medium concentration.

\section{Classification of Synaptic Connections}

Connection type varied between the cell pairs. The types of connections between the cell pairs was recorded in case the type of connection affected the amount of synchronization significantly. The connection between the neurons was studied in control (CM) external conditions. One cell was depolarized to induce action potentials, while the other cell was held at $-65 \mathrm{mV}$ (Figure 5.4). Then the process was repeated for the opposite cell. The connections could either be strong excitatory (s, Figure 5.5 A), weak excitatory (w, Figure 5.5 B), inhibitory (i, Figure 5.5 C) or electrical (e, Figure 5.5 D). Strong excitatory connections had amplitudes greater than $3 \mathrm{mV}$ and less than $10 \%$ failures $\left(n_{c}=11\right)$. Weak excitatory connections included all other excitatory responses including cells with all failures $\left(n_{c}=62\right)$. Inhibitory connections had minimum values that were greater than 3 times the RMS of the noise. The number of inhibitory connections is similar to those expected in hippocampal cultures, about $7 \%\left(n_{c}=10 / 102\right)$ [141]. Electrical connections had small amplitude peaks close to the time of the action potential in the presynaptic 
A
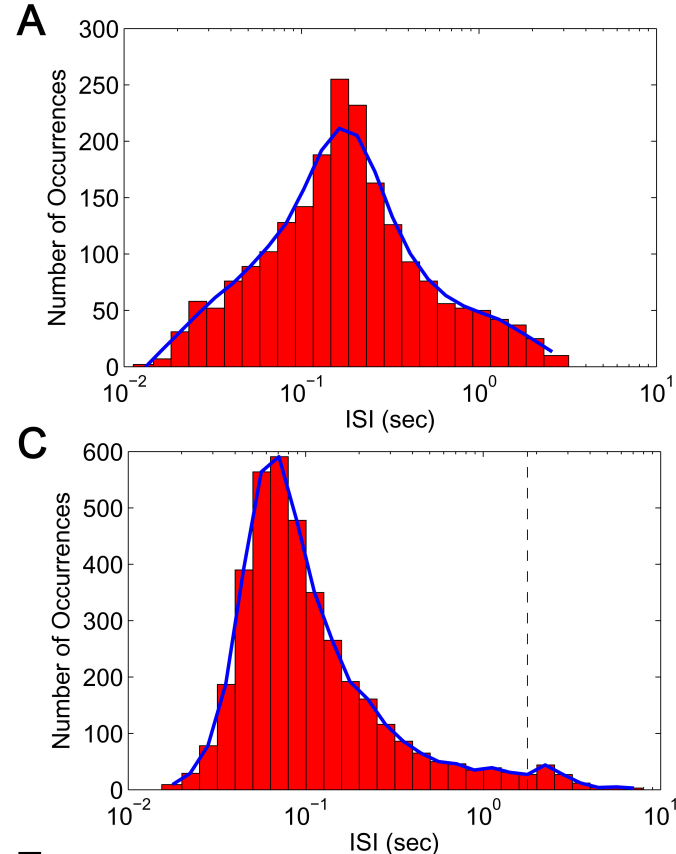

E

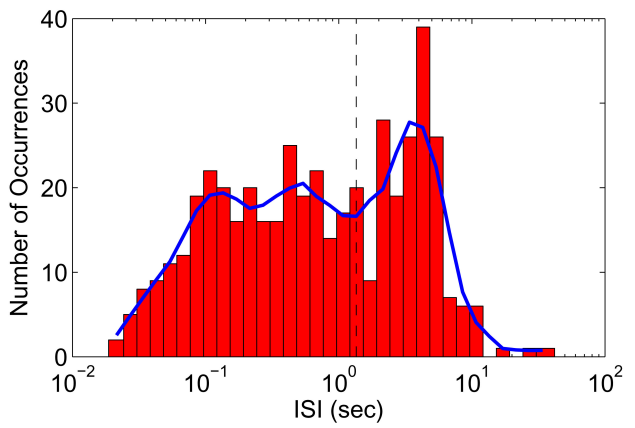

B
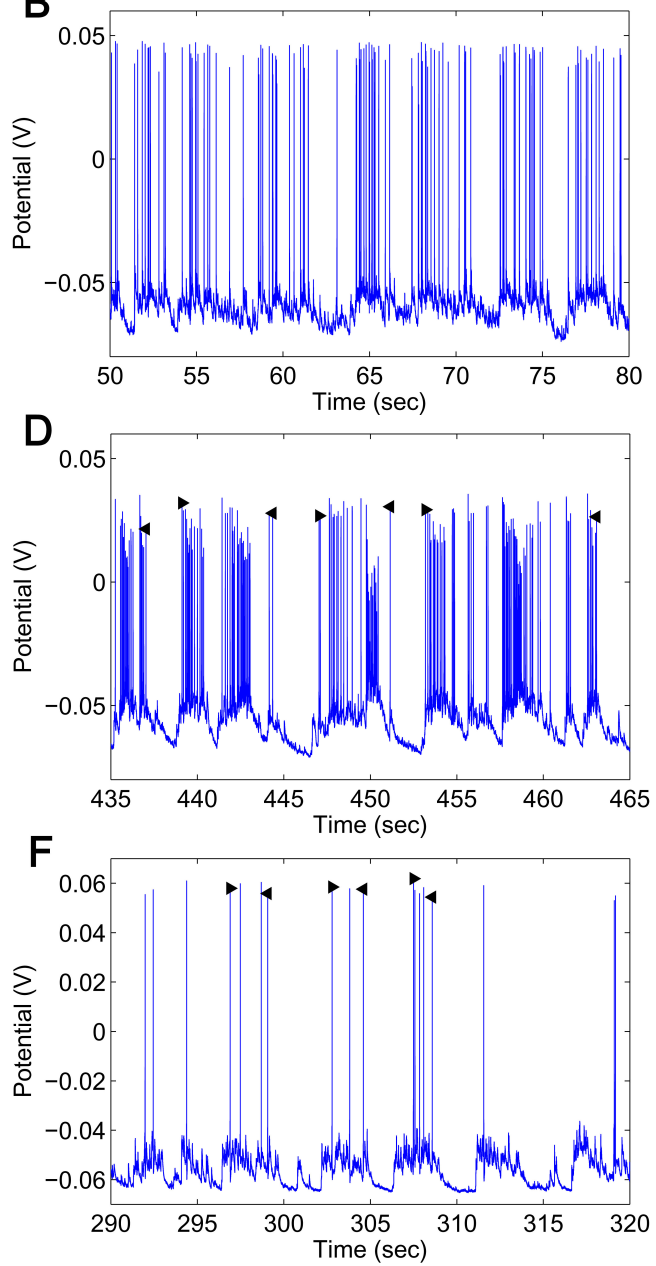

Figure 5.3: Bursts in LM and ZM were identified using the $\log _{10}$ ISI histogram. Blue lines in $\mathrm{A}, \mathrm{C}$ and $\mathrm{E}$ are the smoothed histograms data used to evaluate the presence of peaks. A) One peak in the smoothed logISI histogram signified that no bursts were present in the data. B) Portion of the data for the histogram in A where no bursts were identified by the program. C) LogISI histogram where two peaks were identified and a burst threshold was selected (dashed line). D) Portion of the data for the histogram in C. Arrows demonstrate the start (right arrow) and stop (left arrow) of the bursts as identified by the program. E) LogISI histogram for data with more than two peaks. Burst threshold was identified by the minimum between two peaks as described in Section 5.3.3 (dashed line). F) Portion of the data for the histogram in E. Arrows identify the start (right arrow) and stop (left arrow) of the bursts as identified by the program. 
Table 5.1: Type of connections for grouped and solution-switched experiments

\begin{tabular}{lcccc}
\hline & \multicolumn{4}{c}{ Connection } \\
\cline { 2 - 5 } Experiments & w & s & i & e \\
\hline Grouped & 13 & 3 & 3 & 7 \\
Solution-Switched & 31 & 5 & 5 & 11 \\
\hline
\end{tabular}

cell, followed by a small hyperpolarizing response $\left(n_{c}=19\right)$. The electrical response could be classified as either exciatory, inhibitory or a failure by the detection program, and therefore had to be identified by visual inspection. Each cell in the pair was the presynaptic and postsynaptic cell. Table 5.1 shows the frequency of each connection for all the cell pairs in grouped and solution-switched experiments. The distribution of connections measured in $\mathrm{CM}$ were not statistically significant between the experiments ( $\mathrm{p}=0.83$, Fishers). The average time from the peak of action potential in the presynaptic cell to the peak of EPSP was $17.0 \pm 7.8 \mathrm{~ms}\left(n_{c}=73\right)$.

\subsubsection{Event Synchronization}

The synchronization of the action potentials was measured by using the event synchronization (ES). The maximum window, $\tau_{c}$, was set to $25 \mathrm{~ms}$ based on the average lantency from action potential peak to EPSP peak reported in Section 5.4.1. An example of a pair of cells in CM and a different pair of cells in ZM are shown in Figure 5.6. Action potentials in one cell that were within $25 \mathrm{~ms}$ before an action potential of the other cell are marked with a blue diamond. The strength of synchronization, $Q$, for the $\mathrm{CM}$ pair was 0.13 and the $\mathrm{ZM}$ pair was 0.44 .

All $Q$ for current-clamp pairs are plotted in Figure 5.6. $Q$ in $C M$ was generally low, but a few pairs were highly synchronized $(Q>0.7)$. This demonstrates that naturally connected cells can exhibit high synchronization values, but the cell pairs 

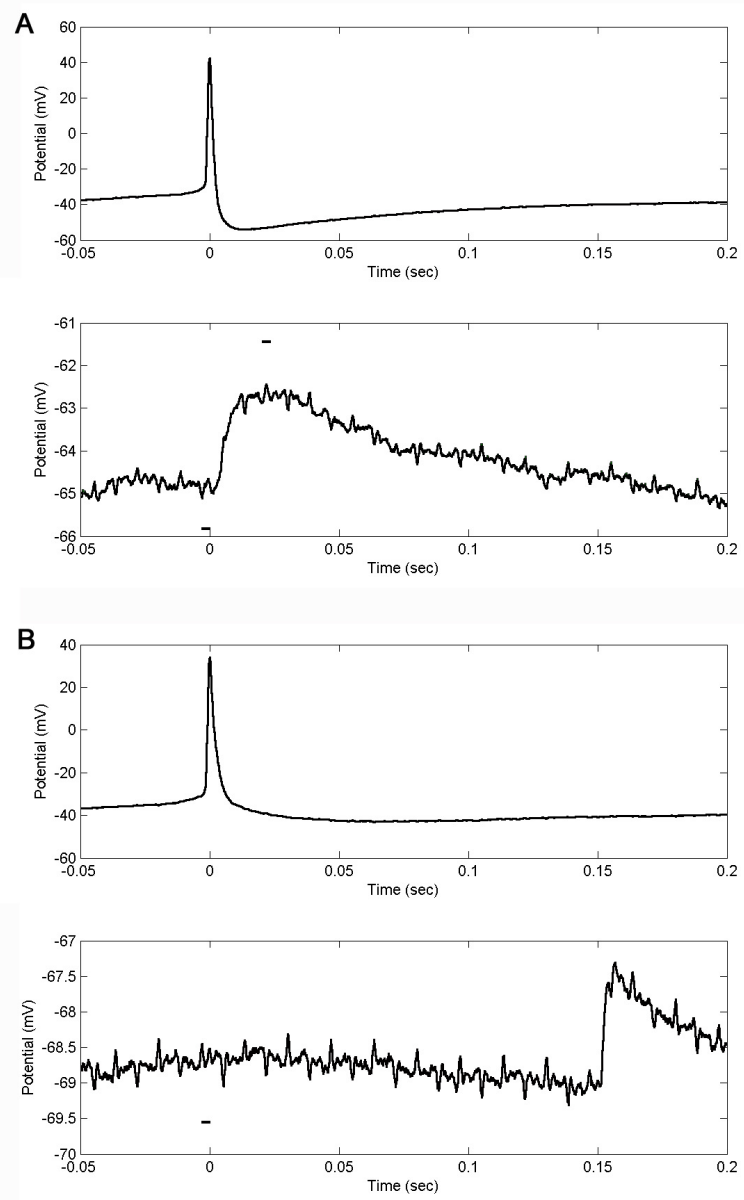

Figure 5.4: Example of the detection of one excitatory response (A) and a failure (B) when analyzing the type of connections between cells. The presynaptic neuron membrane potential is on the top of $A$ and $B$. The postsynaptic neuron membrane potential are on the bottom of A and B. Black lines are $3 \mathrm{~ms}$ long to demonstrate the window over which the potential were averaged to create the local baseline (around 0 seconds, bottom of $A$ and $\mathrm{B}$ ) and the maximum value (around 0.02 seconds, bottom of $\mathrm{A}$ ). The peak of the action potential in the presynaptic cells were set to time $=0 \mathrm{sec}$. The response seen in B bottom around $0.15 \mathrm{sec}$ is an EPSP from a different cell in the culture. 
A

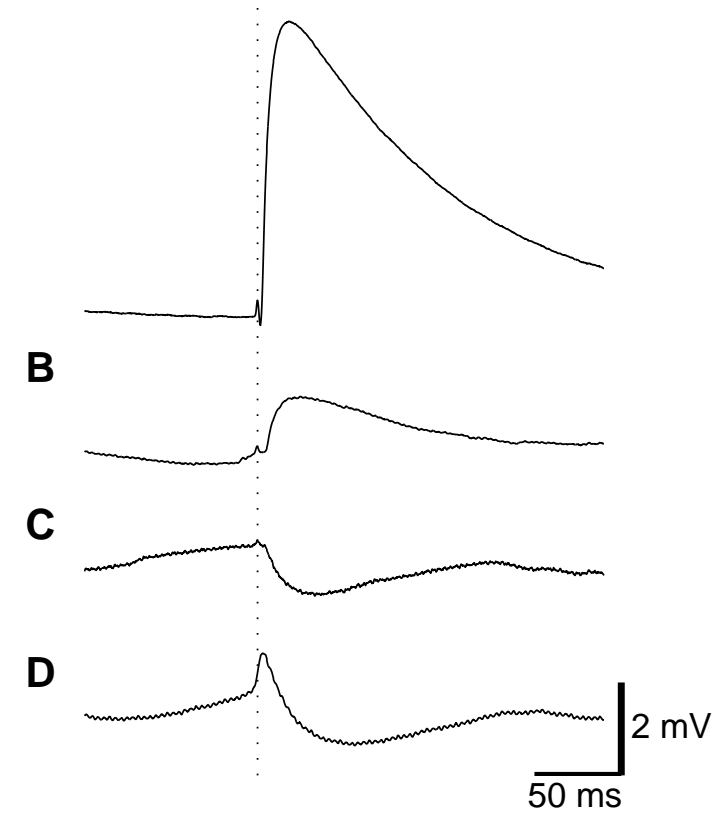

Figure 5.5: Examples of connection types seen in the dual current clamp experiments. A) Strong excitatory connections had an average response with an amplitude greater than $3 \mathrm{mV}$ and less than $10 \%$ failures. B) All other excitatory connections were considered weak. C) Inihibitory connections had minimum values greater than 3 times the RMS noise. Shown are the average IPSP response for one cell. D) Electrical connections were identified visually. The program identifying the connections could classify the electrical response as either excitatory, inhibitory or failure because of their shape and relatively small amplitudes (generally $<1 \mathrm{mV}$ ). Shown are the average for all responses (exitatory, inhibitory and failures) for a cell with an electrical connection. Action potential peak in presynaptic cell occured at the dotted line. Potentials were pinned to $0 \mathrm{mV}$ at the dotted line before averaging.

have lower values of action potential synchronization $(Q<0.3)$. Synchronization values $(Q)$ in LM and ZM were generally higher than those values in $C M$. To examine whether the type of connection affected the synchronization results, the connections of the solution-switched experiments were grouped into weak-weak $\left(n_{p}=13\right)$, strongother $\left(n_{p}=6\right)$, weak-electrical or -inhibitory $\left(n_{p}=4\right)$ and electrical-electrical or - 


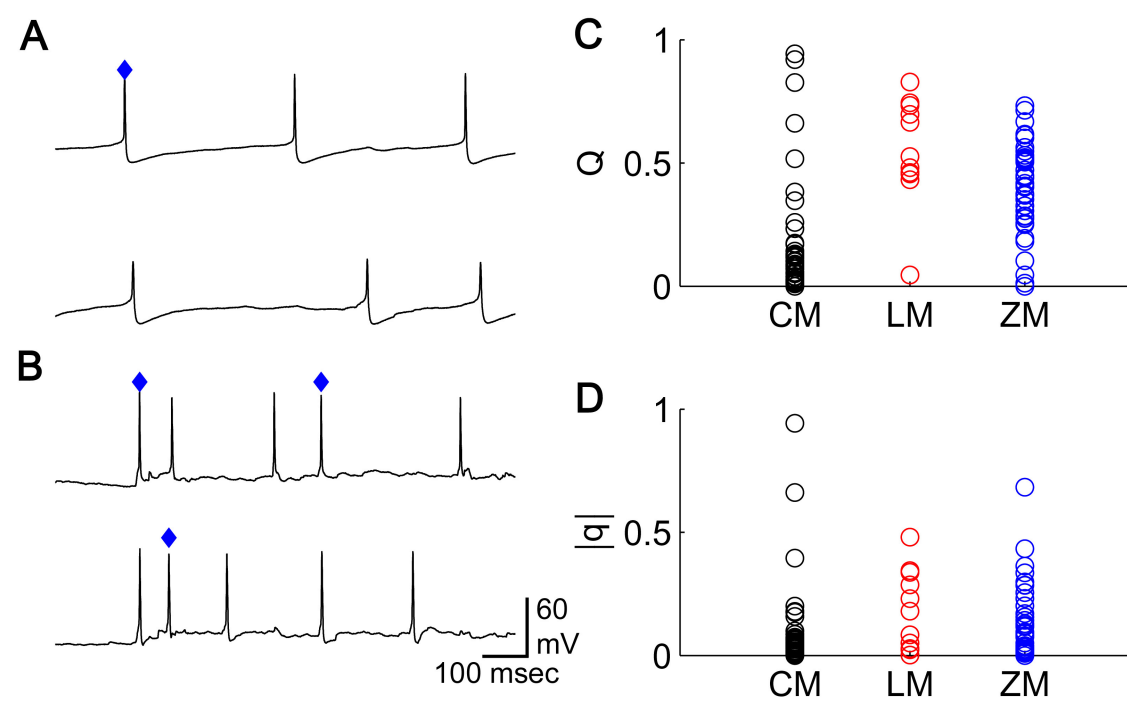

Figure 5.6: Dual whole cell recordings in current-clamp mode. Two traces are shown for $\mathrm{CM}$ conditions where both cells were depolarized to induce recurrent action potentials $(Q=0.13)$. Two traces of two different cells are shown for $\mathrm{ZM}$ conditions $(Q=0.44)$. Blue diamonds indicate action potentials that are synchronized, with the action potential of the leading cell marked. For all dual current-clamp experiments, $Q$ and $|q|$ are shown for the various $\mathrm{Mg}^{2+}$ conditions.

inhibitory $\left(n_{p}=3\right)$. For each of the three $\mathrm{Mg}^{2+}$ concentrations studied, no significant difference was found between the groups of connections (ANOVA, $p>0.05$ ). This is in contrast to modeling and experimental studies where the synchronization is altered based on the type of connections between the cells $[89,125,133]$.

The $|q|$ for all current-clamp pairs are plotted in Figure 5.6. Generally, the $|q|$ values were less than 0.5 , indicating that no preferred order was seen in the pairs (i.e. one cell did not consistently lead). To examine whether the type of connection affected the $|q|$ results, the connections of the solution-switched experiments were grouped into weak-weak $\left(n_{p}=13\right)$, strong-other $\left(n_{p}=6\right)$, weak-electrical or -inhibitory $\left(n_{p}=4\right)$ and electrical-electrical or -inhibitory $\left(n_{p}=3\right)$. For each of the three $\mathrm{Mg}^{2+}$ 
concentrations studied, no significant difference was found between the groups of connections (rmANOVA, $\mathrm{p}>0.05$ ).

Because no significant differences existed, the type of connections were not differentiated and synchronization results were grouped for all analysis. Additionally, since the $|q|$ did not show a significant difference with respect to the types of connections, and most values were $<0.5$ indicating no preferred order, no further analysis was done using $q$ values.

ES is just one possible measure of synchronization and it was chosen because it does not show a bias of synchronization for periods of bursts or single spikes [142], which is important because bursting was not present in all of the data. To insure that the increased frequency of action potentials during bursting activity did not artificially increase $Q$, surrogate pairs of neurons were created from all of the ZM and LM current-clamp recordings. These surrogate pairs mimic dual current-clamp recordings where the cells are not connected. Cells "pairs" were made by randomly assigning cells using two non-repeating permutations. The first minute of each recording was used to create the synchronization values for the pair. Because they are not connected (recorded in different cultures on different days), their synchronization should be very low. An example of surrogate data are shown in Figure 5.7. The top cell was recorded in July 2010 and the second cell was recorded in November 2010. Their synchronization was 0.12 . The average surrogate data synchronization was $0.09 \pm 0.05\left(n_{p}=84\right.$, see Table 5.2). 

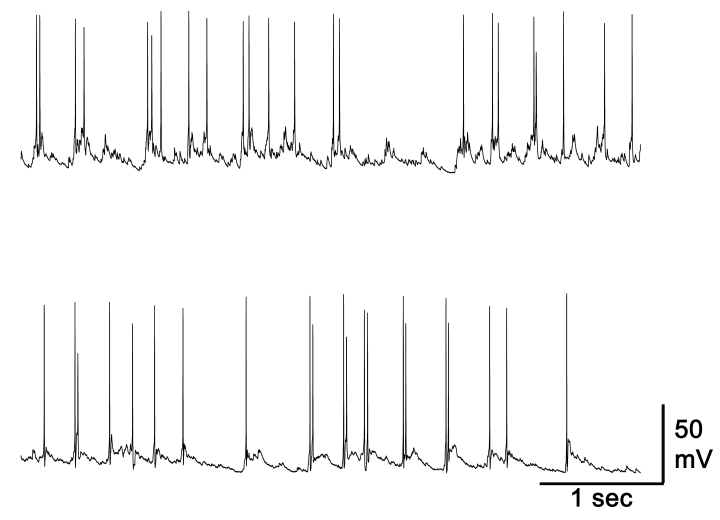

Figure 5.7: Dual whole cell recordings in current-clamp mode for two cell recorded on different days. Circles denote action potentials used to find the synchronization. $Q$ for this pair was 0.12 .

\subsubsection{Effect of Decreased Magnesium on Synchronization}

\section{Grouped Experiments in CM and ZM}

Modeling of synpatically connected neurons with recurrent action potentials (not bursting) have suggested that biologically connected neurons could exhibit synchronized action potentials $[89,133]$. To examine whether synchronization of synaptically connected neurons was enhanced by decreasing extracellular $\mathrm{Mg}^{2+}$, two cells in whole-cell current-clamp mode were recorded under either CM or ZM external conditions.

In $\mathrm{CM}$, both cells were given a suprathreshold current to generate recurrent action potentials (Figure 5.8 CM). Cells in ZM conditions had current injected to adjust the membrane potential to $-65 \mathrm{mV}\left(-64 \pm 8 \mathrm{mV}, n_{c}=16\right.$, Figure $\left.5.8 \mathrm{ZM}\right)$. Synchronization for the data shown in Figure 5.8 were 0.38 and 0.53 for $\mathrm{CM}$ and $Z M$, respectively. For all grouped experiments, $Q$ for $C M$ was $0.13 \pm .19\left(n_{p}=13\right)$ and $0.26 \pm 0.20\left(n_{p}=7\right)$ for ZM (See Table 5.2). The synchronization was not significantly different between the CM and ZM pairs ( $\mathrm{p}=0.16$, $\mathrm{t}$-test).

In the $\mathrm{ZM}$ experiments, it was difficult to measure the strength or type of 
A

CM
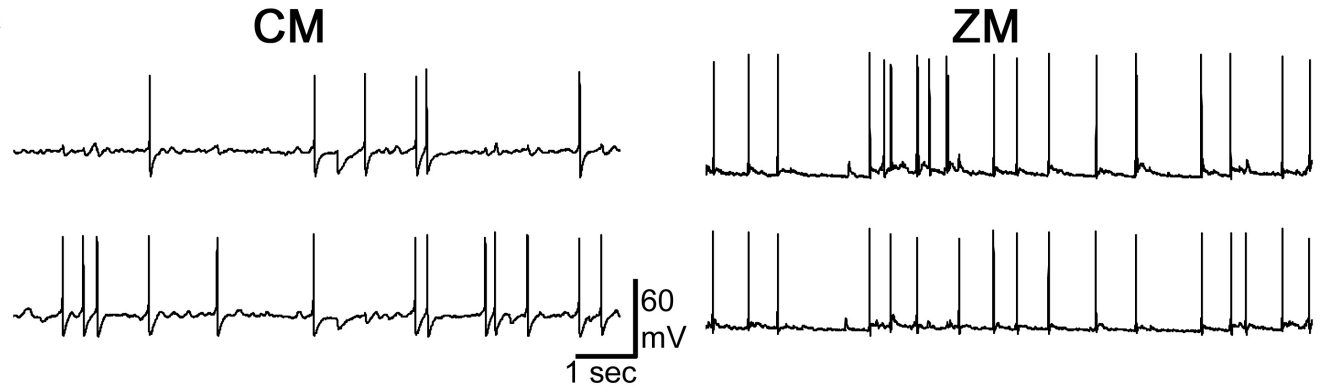

B

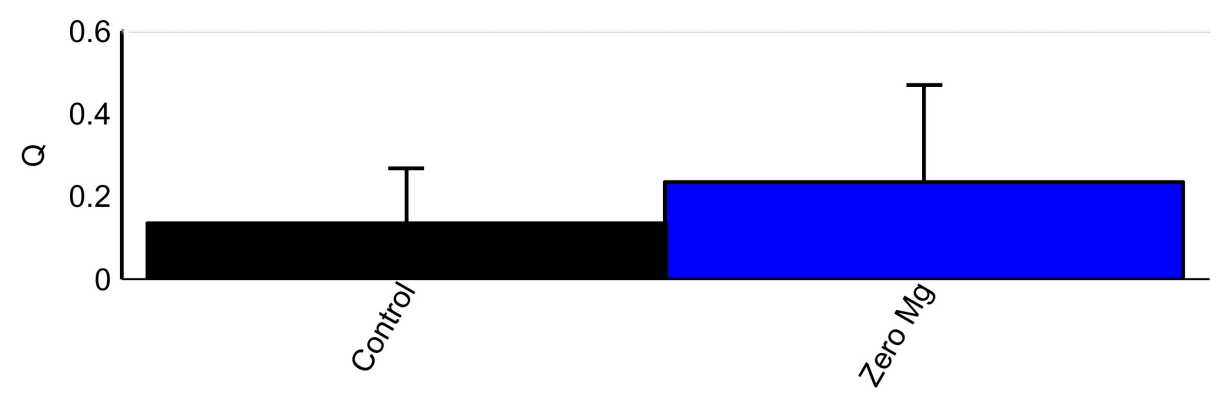

Figure 5.8: Dual whole cell recordings in current-clamp mode. Two traces are shown for CM conditions where both cells were depolarized to induce recurrent action potentials. Two traces of two different cells are shown for $Z M$ conditions. $Q$ was 0.38 for the pair in $C M$ and 0.53 for the pair in ZM. The difference in $Q$ for cells in CM and $Z M$ was not significant ( $\mathrm{p}=0.16$, t-test). In CM 13 cell pairs were recorded and in ZM 7 pairs were recorded.

connection between the cells due to the high amount of activity at $-65 \mathrm{mV}$. No connection data were analyzed for the ZM pairs. The lack of significant change in synchronization could be attributed to any number of differences between the cells measured in $\mathrm{CM}$ and the cells measured in ZM. The cells were different distances apart, part of different cultures with a different number of connections to a variable number of other cells and the type and strength of the connections in ZM could not be determined accurately. In order to keep these variables the same in CM and ZM, solution-switch experiments were performed where the solution was exchanged from $\mathrm{CM}$ to $\mathrm{ZM}$ while maintaining the dual patch clamp so cell pairs could be measured in both conditions.

While the result was not significant, $Q$ did increase from CM to ZM. It would be 
possible that the increase in $Q$ would not be significantly different in the solutionswitch experiments. Solution-switch experiments were also performed by exchanging CM to LM conditions. The addition of LM can demonstrate a trend in synchronization even if the change in synchronization is not significant.

\section{Solution-Switch Experiments}

In the grouped experiments, the type of connection could not be determined for the pairs of neurons in ZM. This may have been the reason the change in synchronization was not significant. To keep the type of connection the same in CM and ZM, the same cell pairs were measured in CM and LM or ZM. The solution was switched from CM to ZM or LM using a bath perfusion system. LM conditions were studied to further examine the relationship between action potential synchronization and synaptic strength.

Two cells were whole-cell patch-clamped in current-clamp mode in CM. The types of connections between the cells were measured as previously described. Both cells were depolarized with a suprathreshold current to generate action potentials. The external medium was exchanged to either ZM or LM. Recordings for two cells in $\mathrm{CM}$ and the same two cells in ZM are shown in Figure 5.9 A. Synchronization for the pair in $\mathrm{CM}$ was 0.02 and in $\mathrm{ZM}$ was 0.62 . Figure $5.9 \mathrm{C}$ shows that the synchronization was significantly changed from control to $\mathrm{ZM}\left(n_{p}=17, \mathrm{p}=0.00007\right.$, paired t-test, see Table 5.1). Recordings for two cells in CM and the same two cells in LM are shown in Figure 5.9 B. Synchronization for the pair in CM was 0.17 and it increased in LM to 0.83 . Figure $5.9 \mathrm{C}$ shows that the synchronization was significantly changed from control to $\operatorname{LM}\left(n_{p}=9, \mathrm{p}=0.00007\right.$, paired $\mathrm{t}$-test, see Table 5.1). Interestingly, the synchronization was higher in LM than in ZM; however, the synchronization difference between LM and ZM was not significant (rmANOVA, 
A

CM
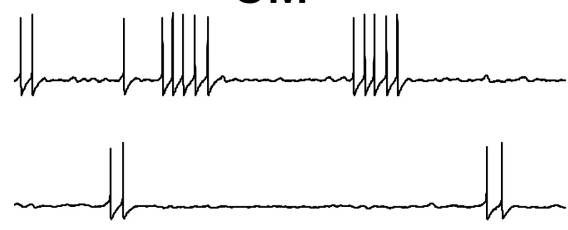

CM
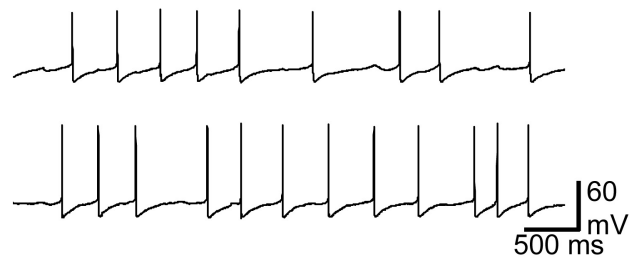

B

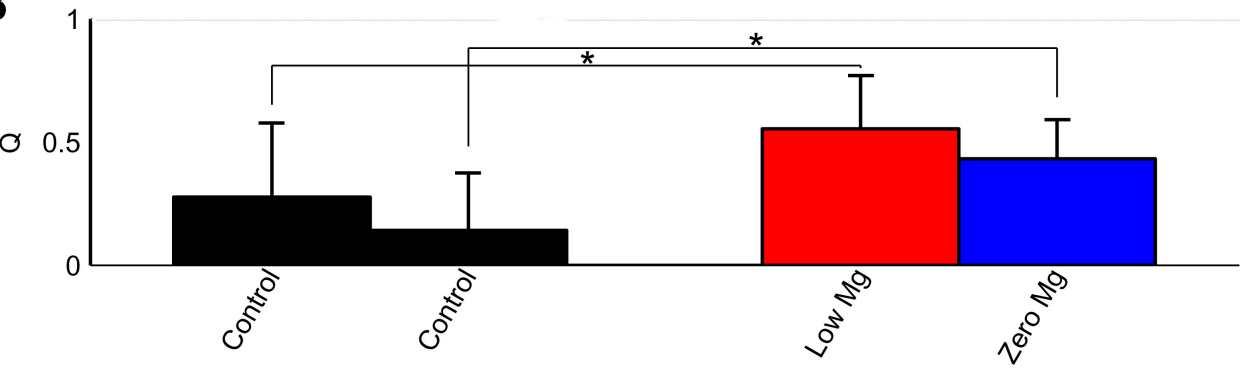

Figure 5.9: Dual whole cell recordings in current-clamp mode under $C M$ conditions (A, left) and ZM conditions (A, right). Both cells were depolarized in $\mathrm{CM}$ to induce recurrent action potentials. Synchronization for the pair under $\mathrm{CM}$ was 0.02 and under $\mathrm{ZM}$ the synchronization was increased to 0.62 . For pairs solution-switched from $\mathrm{CM}$ to $\mathrm{ZM}$, synchronization increased significantly from $0.14 \pm 0.23$ to $0.26 \pm 0.20$, respectively $\left(n_{p}=17, \mathrm{p}=0.00007\right.$, paired t-test). Dual whole cell recordings in current-clamp mode under $\mathrm{CM}$ conditions (A, left) and LM conditions (A, right). Both cells were depolarized in $\mathrm{CM}$ to induce recurrent action potentials. Synchronization for the pair under CM was 0.17 and under LM the synchronization was increased to 0.83. For pairs solution-switched from CM to LM, synchronization increased significantly from $0.27 \pm 0.30$ to $0.55 \pm 0.22$, respectively $\left(n_{p}=9, \mathrm{p}=0.00007\right.$, paired t-test). *significant, $\mathrm{p}<0.05$.

post-hoc Tukey).

Ideally, to examine the relationship between synchronization and synaptic strength, the synchronization and change in synaptic strength would be measured in the same cell pair under CM and ZM (or LM). However, the same problems that made it difficult to measure the type of connection in $\mathrm{ZM}$ for the grouped 
experiments also affect the ability to detect change in synaptic strength in the solution-switch experiments. Action potentials were present in both the presynaptic and postsynaptic cells and they often occur near simultaneously, obscuring the EPSP responses to those action potentials. Firing of multiple other cells in the culture created many EPSPs in the postsynaptic cell. The EPSPs in response to the presynaptic (recorded) cell could not be distinguished from other EPSPs. The membrane potential was highly variable because of the temporary depolarizations underlying the ZM bursting. The amplitudes of EPSPs depend on the initial membrane potential $[1,16]$. The use of CM to LM solution-switched experiments did not alleviate any of these issues.

To measure the synaptic strength, dual whole-cell patch-clamp experiments were performed with one cell in current-clamp mode (presynaptic cell) and the other cell in voltage-clamp mode (postsynaptic cell). Action potentials in the postsynaptic cell were blocked by addition of lidocaine to the internal solution so that only EPSCs were measured. In voltage-clamp mode, the membrane potential is held relatively constant making the measured amplitudes during bursting slightly more accurate. Because the specific response due to activity in the presynaptic cell cannot be distinguished from activity due to other cells in the culture, two measures of synaptic strength were used: the method of failures which depends on responses relative to the presynaptic cell and the increase of EPSC amplitudes which depends on all EPSCs recorded in the postsynaptic cell.

\subsubsection{Effect of Decreased Magnesium on Synaptic Strength}

Decreasing $\mathrm{Mg}^{2+}$ primarily increases synaptic strength by unblocking NMDA receptors [135]. Quantal analysis was using to quantify the change in synaptic strength when $\mathrm{Mg}^{2+}$ was decreased in the external solution. Increasing the connections 
between cells can also increase the synchronization [143]. Ideally, the change in the synaptic strength would be measured in the same cell pairs that synchronization was measured. The presence of action potentials in both cells in LM and ZM with the increase of EPSPs from other cells in the culture made direct quantal analysis on the EPSPs difficult. Instead, one cell was whole-cell patch-clamped in currentclamp mode (presynaptic cell) and another cell was whole-cell patch-clamped in voltage-clamp mode (postsynaptic cell). The postsynpatic cell had lidocaine in the internal solution to block action potentials. The presynaptic cell was given a suprathreshold current in CM conditions. The solution was exchanged using a perfusion system to LM external solution and then to ZM. The presynaptic cell in LM or ZM was injected with current to keep the minimum membrane potential around $-65 \mathrm{mV}$ or given a suprathreshold current to cause more action potentials.

Action potentials of the presynaptic cell were identified and the cell pairs were separated by the strength of their excitatory connections in CM, either strong or weak. The reversal potential for $\mathrm{Cl}^{-}$was $-68 \mathrm{mV}$ for these conditions, meaning inhibitory connections would cause responses in the opposite direction of the excitatory connections. Therefore, even though no $\mathrm{GABA}_{\mathrm{A}}$ blockers were used in the experiments, the responses analyzed below are all considered excitatory.

An example of two cells with a strong excitatory connection is shown in Figure 5.10. All EPSC amplitudes in the first two minutes of the recordings were used to create amplitude histograms. The histograms were fit with multiple Gaussian curves. Peaks were at 20.9, 47.8, 67.8 and 99.6 pA in control, 20.0, 31.6, 49.3 and 100.4 pA in LM and 22.4, 41.3, 54.0, 92.0 and $139.5 \mathrm{pA}$ in ZM. As the concentration of $\mathrm{Mg}^{2+}$ was decreased to $\mathrm{ZM}$ a new population of high amplitude EPSCs emerged with amplitudes around $140 \mathrm{pA}$, demonstrating that the mean quantal content increased. In the pairs with a strong connection, $m_{f}$ decreased from control to LM, 

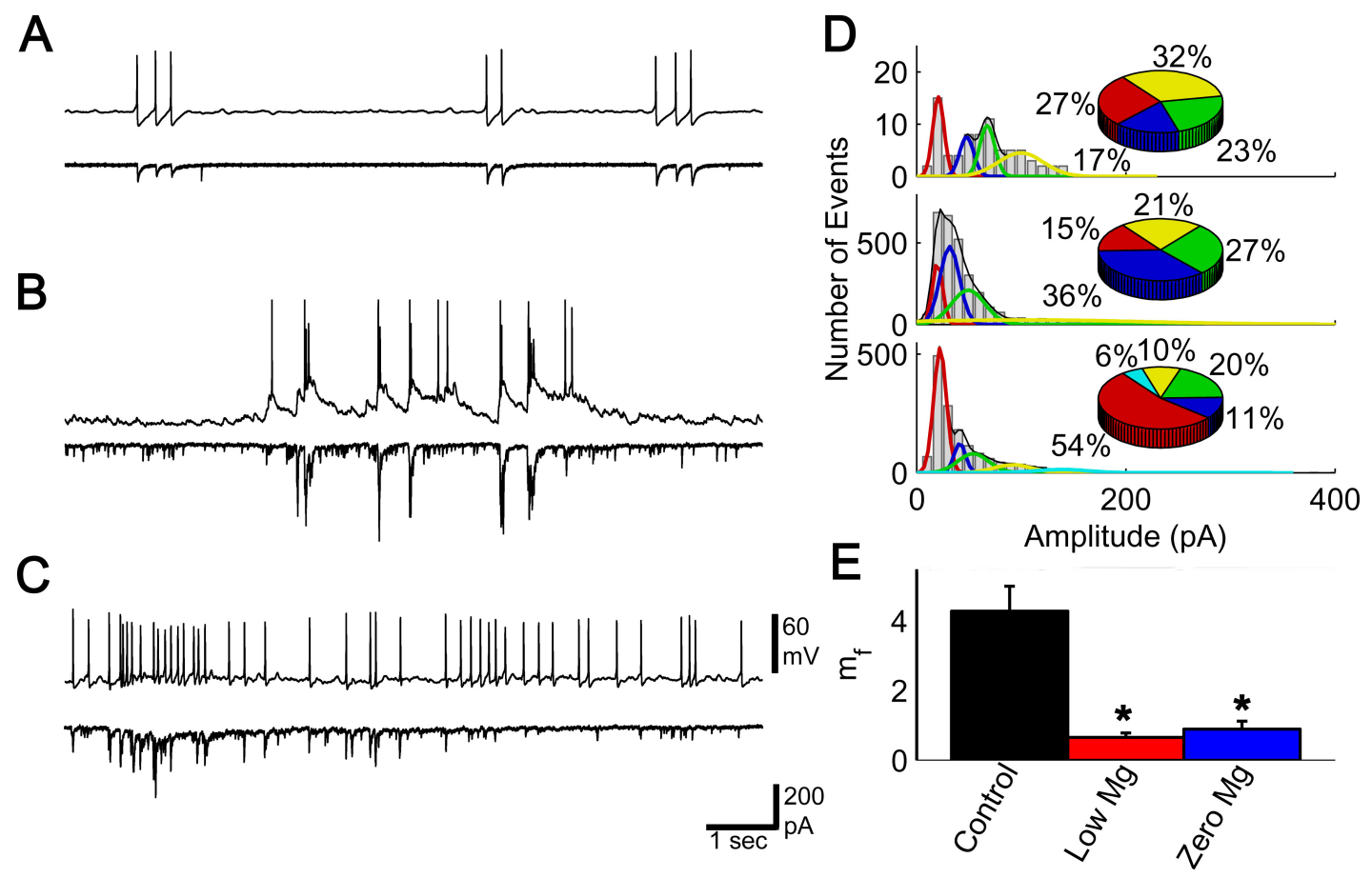

Figure 5.10: Connectivity was decreased from $\mathrm{CM}$ to LM and increased from LM to ZM in a neuron pair with a strong connection under CM conditions. One cell in current clamp mode (A, B, C, top) and one cell in voltage clamp mode (A, B, C, bottom) were measured in CM (A), LM (B) and ZM (C) external conditions. D) Amplitude distributions for all EPSCs for the cell in voltage clamp mode under CM (top), LM (middle) and ZM (bottom) conditions. Amplitudes increased slightly from CM to LM and ZM. Fits of the amplitudes reveal new populations of amplitudes as magnesium was decreased. Corresponding pie charts demonstrate the relative amount of amplitudes at each peak value. E) $m_{f}$ was calculated for responses based on the action potentials of the cell in current clamp mode. The number of failures increased from control to LM and then decreased slightly to ZM. $m_{f}$ for CM was significantly higher than LM and ZM ( $<<0.0001$ for both, $n_{p}=4$, repeated measures ANOVA, post-hoc Tukey method). *significant, $\mathrm{p}<0.05$.

but then increased slightly from LM to ZM (Figure 5.10 C). Both LM and ZM were significantly different from $\mathrm{CM}\left(\mathrm{p}<0.0001\right.$ for both, $n_{p}=4$, rmANOVA, post-hoc Tukey method).

An example of two cells with a weak excitatory connection is shown in Figure 5.10. Again, amplitude histograms were made from the first two minutes of the 
recordings and fit with multiple Gaussian curves. For the weakly connected neurons, peaks were at 20.7 and $43.5 \mathrm{pA}$ in $\mathrm{CM}, 20.8,39.7,63.1,113.2,176.3$ and $238.6 \mathrm{pA}$ in $\mathrm{LM}$ and $20.7,34.2,55.9,80.1,132.7$ and $256.0 \mathrm{pA}$ in $\mathrm{ZM}$. As the concentration of $\mathrm{Mg}^{2+}$ was decreased to ZM a new population of high amplitude EPSCs emerged with amplitudes around $140 \mathrm{pA}$, demonstrating that the mean quantal content increased. For pairs with a weak connection, $m_{f}$ was increased as $\mathrm{Mg}^{2+}$ was decreased (Figure $5.11 \mathrm{C})$. However, only the ZM was significantly different from control $(\mathrm{p}=0.026$, $n_{p}=4$, repeated measures ANOVA, post-hoc Tukey method). Four new populations emerged in the LM conditions and were also present in the ZM conditions. This suggests that for the network activity for the strong and weak excitatory connected neurons, the synaptic strength increased, even thought the direct connection in the strong pairs decreased.

\subsubsection{Effect of Antiepileptic Drugs on Synchronization}

Antiepileptic drugs (AEDs) can treat seizures through a variety of mechanisms; they can attenuate bursting activity and decrease synaptic strength. Three AEDs were applied to cells in $\mathrm{ZM}$, an in vitro seizure-like condition. The drugs were chosen based on their mechanisms of action. Phenytoin (PHT) stops recurrent action potentials by blocking $\mathrm{Na}^{+}$channels $[144,145]$, but does not alter synaptic strength. GYKI-52466 (GYKI) blocks AMPA and kainate receptors [146], which play a role in the bursting behavior of ZM [135]. LEV has multiple mechanisms of action $[147,148]$, and has been shown in some in vitro models to decrease synchronization [33]. Levetiracetam (LEV) has been shown to bind to synaptic vesicle protein 2 (SV2) [149]. Loss of SV2 reduces release probability for a train of action potentials [150], which means that synaptic strength is decreased specifically during high frequency stimulations, like bursting activity. 

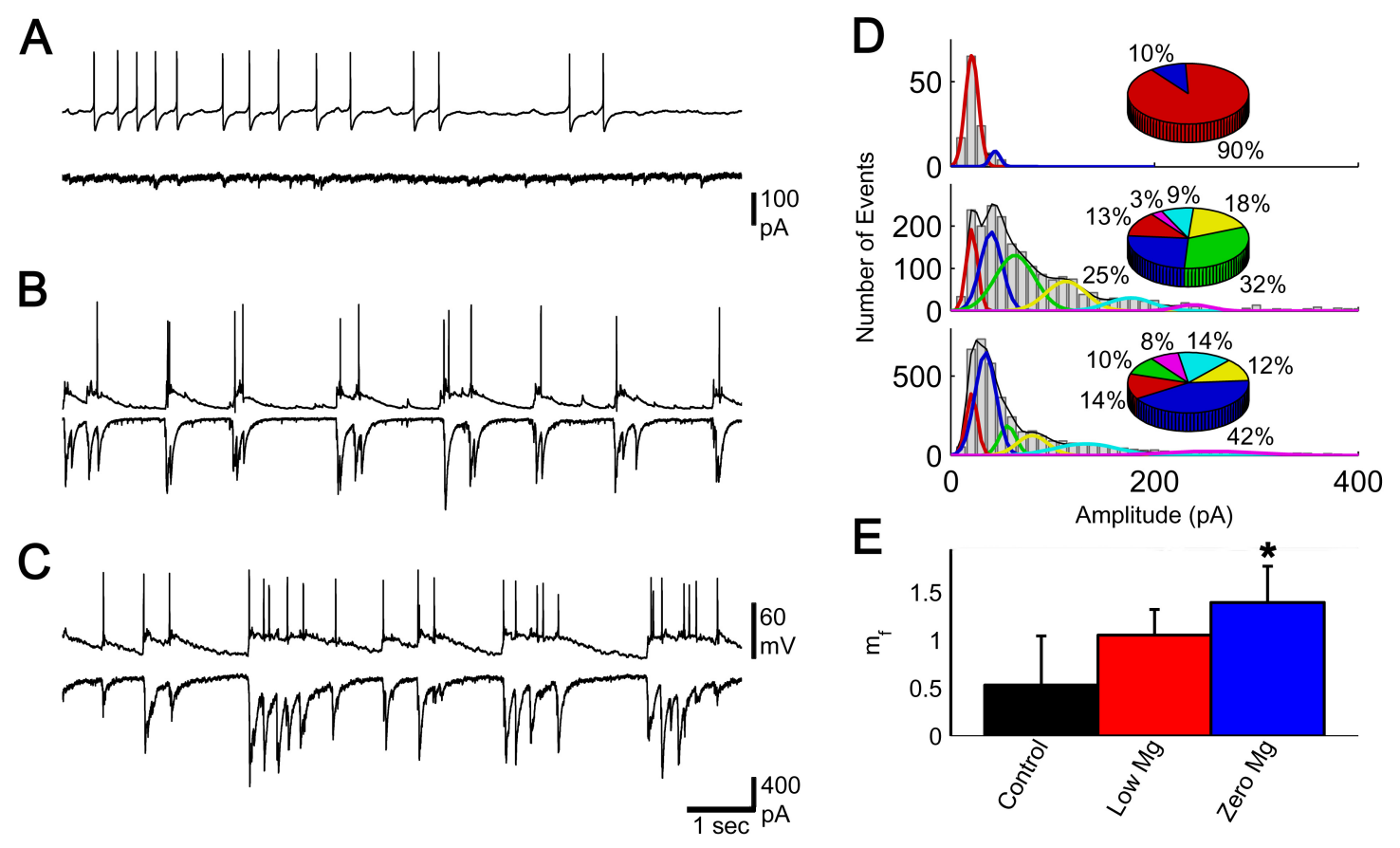

Figure 5.11: Connectivity was increased from CM to LM and from LM to $\mathrm{ZM}$ in a pair with a weak connection under CM conditions. One cell in current clamp mode $(\mathrm{A}, \mathrm{B}, \mathrm{C}$, top) and one cell in voltage clamp mode $(\mathrm{A}$, $B, C$, bottom) were measured in control (A), LM (B) and ZM (C) external conditions. D) Amplitude distributions for all EPSCs for the cell in voltage clamp mode under CM (top), LM (middle) and ZM (bottom) conditions. Amplitudes increased from CM to LM and ZM. Fits of the amplitudes reveal new populations of amplitudes as magnesium was decreased. Corresponding pie charts demonstrate the relative amount of amplitudes at each peak value. E) $m_{f}$ was calculated for responses based on the action potentials of the cell in current clamp mode. The number of failures decreased from CM to LM and then decreased slightly from LM to ZM. $m_{f}$ increased from CM to LM to ZM, with CM and ZM being significantly different ( $\mathrm{p}=0.026, n_{p}=4$, repeated measures ANOVA, post-hoc Tukey method). *significant, $\mathrm{p}<0.05$.

Dual whole-cell current-clamp recordings were made for cells in CM, then the solution was exchanged to ZM and finally in ZM + AED. AED was either $40 \mu \mathrm{M}$ PHT, $75 \mu \mathrm{M}$ GYKI or 50-100 $\mu \mathrm{M}$ LEV. Concentrations used were similar those found elsewhere $[27,151,152]$ and were adjusted so that spontaneous activity was not abolished at $-65 \mathrm{mV}$. Cells in CM were given a suprathreshold current to 
create action potentials. Some cell pairs in these experiments were not able to fire recurrent action potentials and the synchronization for those experiments were set to 0 (GYKI, $n_{p}=1$; LEV, $n_{p}=1$ ). In ZM and ZM + AED conditions, cells were injected with current to adjust the minimum membrane potential of each cell to $\sim-65 \mathrm{mV}$.

An example of two cells in CM are shown in Figure 5.12 A $(Q=0.10)$ and the same cells are shown in $\mathrm{ZM}(Q=0.62)$. $\mathrm{ZM}+\mathrm{PHT}$ stopped bursting activity and synchronization was increased to $Q=0.78$ (Figure $5.12 \mathrm{~A}$ bottom). Interevent intervals (IEIs) are plotted in Figure 5.12 B in CM (black), ZM (blue) and ZM + PHT (green) for the second cell (bottom traces of CM, ZM and ZM + PHT in A). The cumulative distributions of IEIs were significantly different for all conditions $(\mathrm{p}<0.0001, \mathrm{~K}-\mathrm{S}$ test $)$. For four pairs, average synchronization was increased from $\mathrm{CM}$ to $\mathrm{ZM}(0.06 \pm 0.04$ to $0.57 \pm 0.05)$, but addition of PHT did not affect the synchronization $(0.56 \pm 0.06$, Figure $5.12 \mathrm{C})$. The increase in synchronization from $\mathrm{CM}$ to $\mathrm{ZM}$ was significant, but not the change from $\mathrm{ZM}$ to $\mathrm{ZM}+\mathrm{PHT}(\mathrm{p}=0.98$, rmANOVA, post-hoc Tukey).

Synchronization of the two cells in CM shown in Figure 5.13 A was 0 and for the same cells in ZM was 0.35. Surprisingly, the addition of GYKI had an effect similar to that of PHT in that the bursting was attenuated (Figure $5.13 \mathrm{~A}$, bottom), but the synchronization for ZM + GYKI was increased to 0.90 . The cumulative distributions of IEIs were significantly different for all conditions (Figure 5.13 B, $p<0.0001$, K-S test). For four pairs, average synchronization was increased from $\mathrm{CM}$ to $\mathrm{ZM}(0.03 \pm 0.04$ to $0.50 \pm 0.16)$, and addition of GYKI also increased the synchronization, but not significantly $(0.69 \pm 0.20$, Figure $5.13 \mathrm{C}, \mathrm{p}=0.20$, rmANOVA, post-hoc Tukey). This was unexpected because GYKI decreases the strength of the synaptic connections and should have decreased the ability of the cells to synchronize. 
A
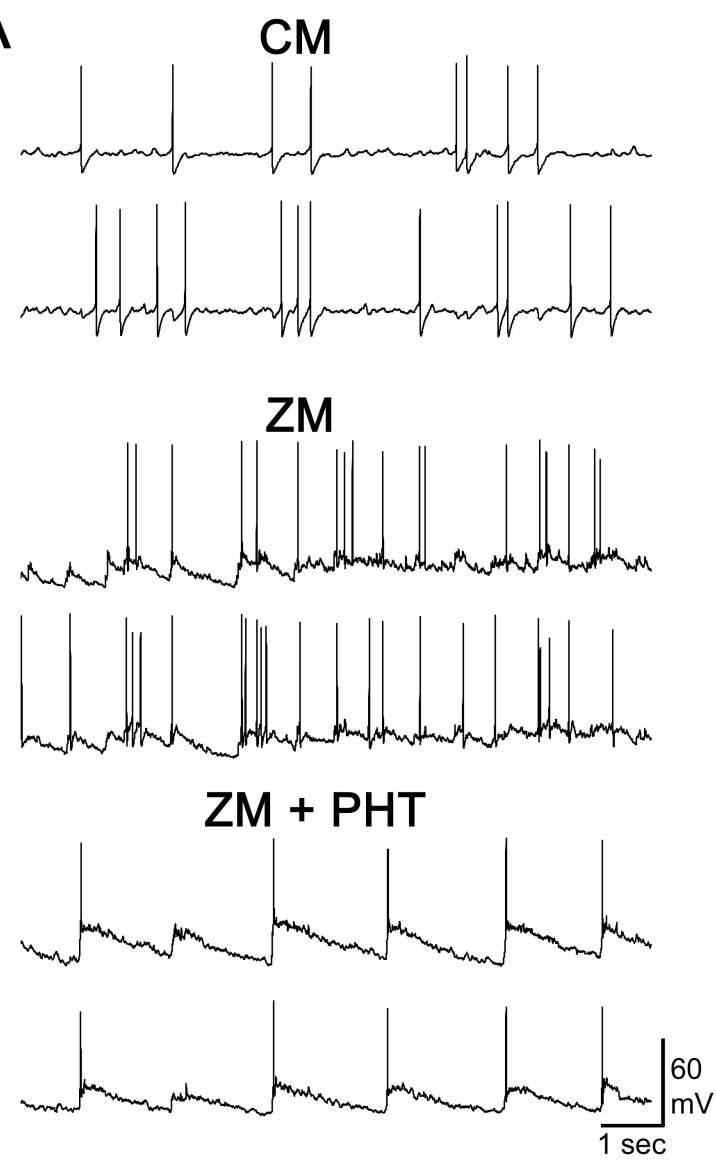

B
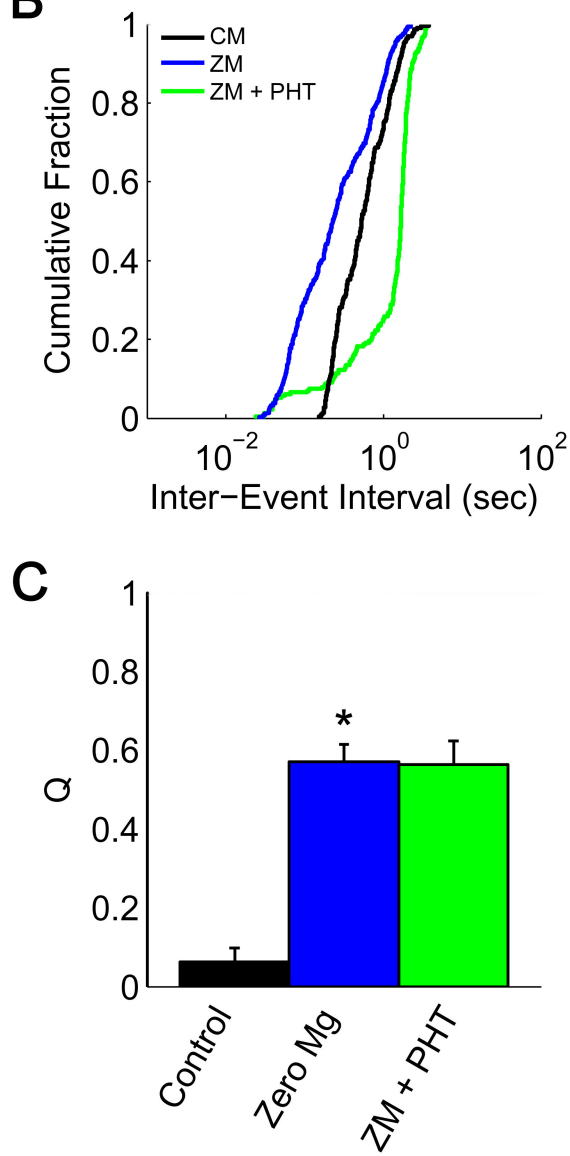

Figure 5.12: Dual whole cell recordings in current-clamp mode. A Cell 1 (top) and Cell 2 (bottom) under CM, ZM and ZM $+40 \mu \mathrm{M}$ PHT conditions. Both cells were depolarized in CM to induce recurrent action potentials. B) The Inter-Event Interval (IEI) cumulative distribution for one cell shows that ZM (blue line) had a greater amount of small IEIs than CM ( $<<0.0001$, $\mathrm{K}-\mathrm{S}$ test), and addition of PHT shifted the distribution to larger IEIs (blue line, $\mathrm{p}<0.0001$, K-S test). C) Although PHT changed the IEI distribution significantly, no significant change was seen in the synchronization $(\mathrm{p}=0.98$, rmANOVA, post-hoc Tukey). *significant, $\mathrm{p}<0.05$. 
A

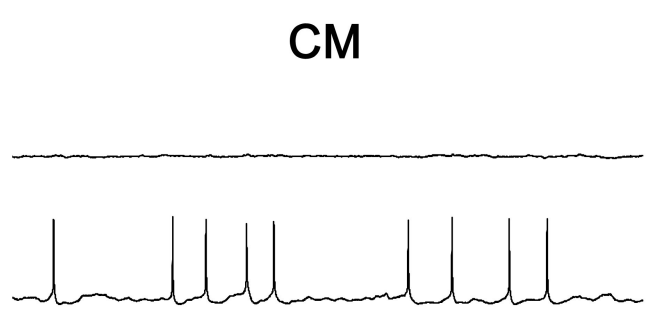

ZM

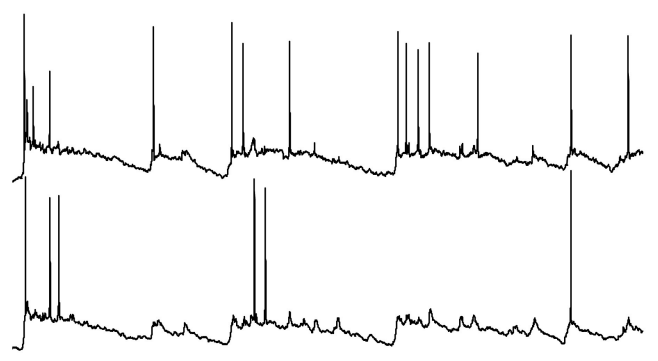

B
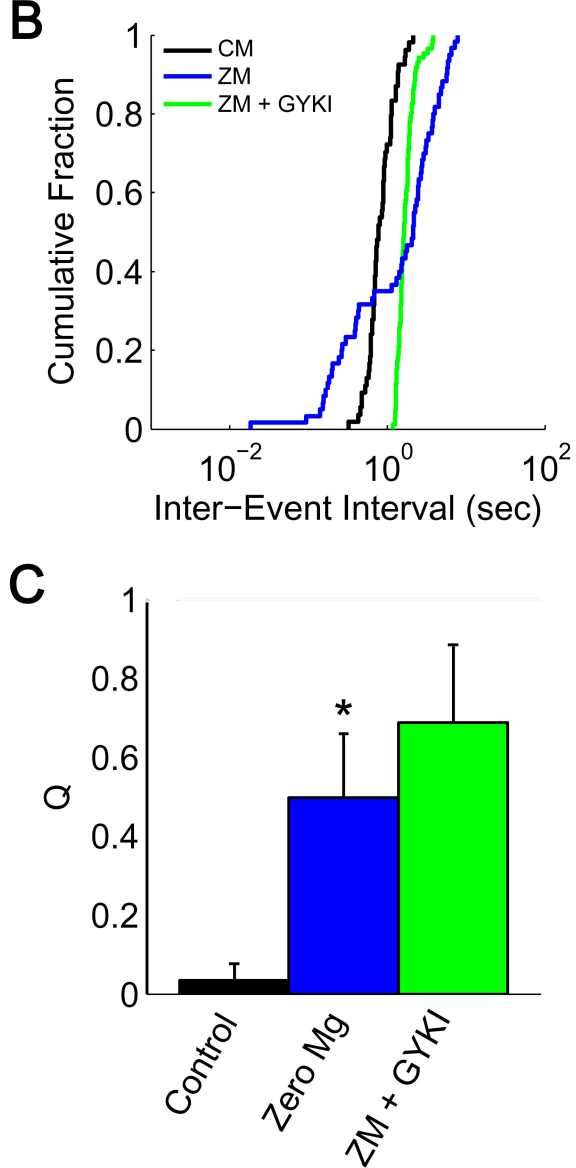

Figure 5.13: Dual whole cell recordings in current-clamp mode. A) Cell 1 (top) and Cell 2 (bottom) under CM, ZM and ZM + $75 \mu \mathrm{M}$ GYKI conditions. Both cells were depolarized in $\mathrm{CM}$ to induce recurrent action potentials. B) The Inter-Event Interval (IEI) cumulative distribution for one cell shows that ZM (blue line) had a greater amount of small IEIs than control $(\mathrm{p}<0.0001, \mathrm{~K}-$ $S$ test), and addition of GYKI shifted the distribution left to larger IEIs (green line, $\mathrm{p}=0.0001, \mathrm{~K}-\mathrm{S}$ test). C) Although GYKI changed the IEI distribution significantly, no significant change was seen in the synchronization from $\mathrm{ZM}$ to $\mathrm{ZM}+\mathrm{GYKI}$ ( $\mathrm{p}=0.20$, rmANOVA, post-hoc Tukey). *significant, $\mathrm{p}<0.05$. 
Two cells in CM, ZM and ZM + LEV are shown in Figure 5.14 A. Synchronization for $\mathrm{CM}$ was 0 and in $\mathrm{ZM}$ was 0.18 . Switching to $\mathrm{ZM}+\mathrm{LEV}$ showed little alteration in bursting activity (Figure 5.14 B); the synchronization for ZM + LEV was slightly increased to 0.21 . The cumulative distributions of IEIs were significantly different for all conditions (Figure $5.14 \mathrm{~B}, \mathrm{p}=0.0001$, K-S test). For four pairs, average synchronization was increased from CM to ZM $(0.00 \pm 0.01$ to $0.25 \pm 0.04)$. Addition of LEV decreased the synchronization, but not significantly $(0.19 \pm 0.07$, Figure 5.14 C, $\mathrm{p}=0.32$, rmANOVA, post-hoc Tukey). The synchronization in ZM was lower for cells pairs used in ZM + LEV than in the PHT and GYKI experiments (Table 5.2). This may have had an effect on how well LEV could desynchronize the pairs of cells if the cells were not very synchronized initially.

\subsection{Discussion}

This chapter examined the relationship between synchronization of action potentials and synaptic strength. It has been suggested that synchronized behavior is increased as the synaptic strength is increased $[135,143]$. The strength of connections between neurons were examined as external $\mathrm{Mg}^{2+}$ was decreased. $\mathrm{Mg}^{2+}$ strengthens synaptic connections primarily by unblocking NMDA receptors $[34,135]$. The effect of decreasing $\mathrm{Mg}^{2+}$ on synaptic strength was analyzed using quantal analysis.

Synchronization of the bursting activity has been examined using cross-correlation [28-30], field potentials [31-33] and by visual inspection [34-38]. In this chapter, the event synchronization (ES) was used to quantify the amount of action potential synchronization between two cells in various external concentrations of $\mathrm{Mg}^{2+}$. ES is just one possible measure of synchronization. Compared to other synchronization measures, ES has similar results in distinguishing levels of synchronization and 
A

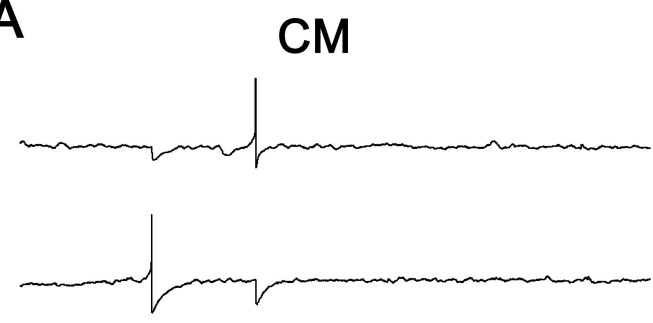

ZM
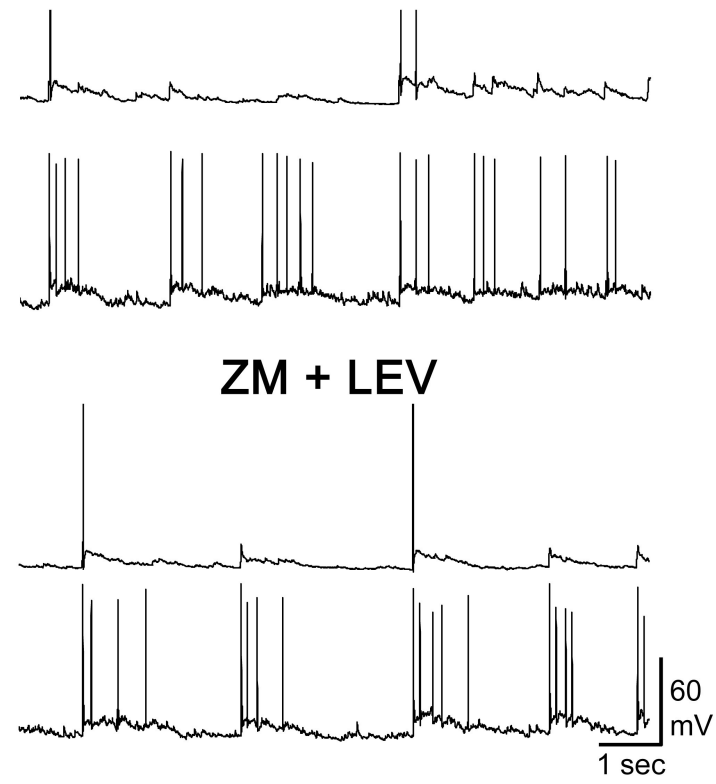

B

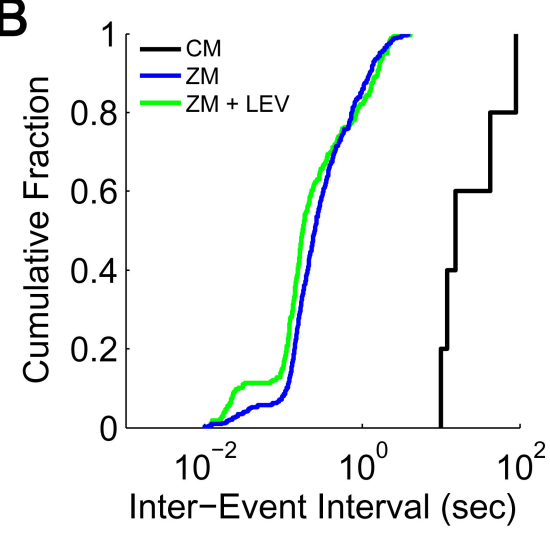

C

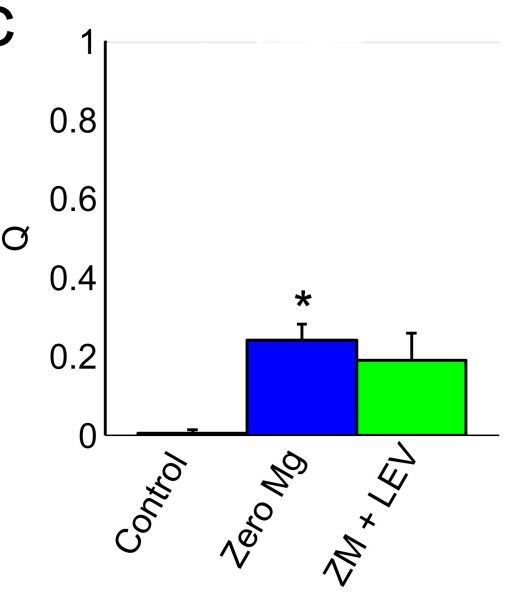

Figure 5.14: Dual whole cell recordings in current-clamp mode. A) Cell 1 (top) and Cell 2 (bottom) under CM, ZM and ZM + 50-100 $\mu \mathrm{M} \mathrm{LEV}$ conditions. Both cells were depolarized in $\mathrm{CM}$ to induce recurrent action potentials. B) The Inter-Event Interval (IEI) cumulative distribution shows for one cell that ZM (blue line) had a greater amount of small IEIs than control (K-S test), and addition of LEV created slightly more IEIs (green line, $\mathrm{p}=0.0001, \mathrm{~K}-\mathrm{S}$ test). C) Although LEV changed the IEI distribution significantly, no significant change was seen in the synchronization $(\mathrm{p}=0.32$, rmANOVA). *significant, $\mathrm{p}<0.05$. 


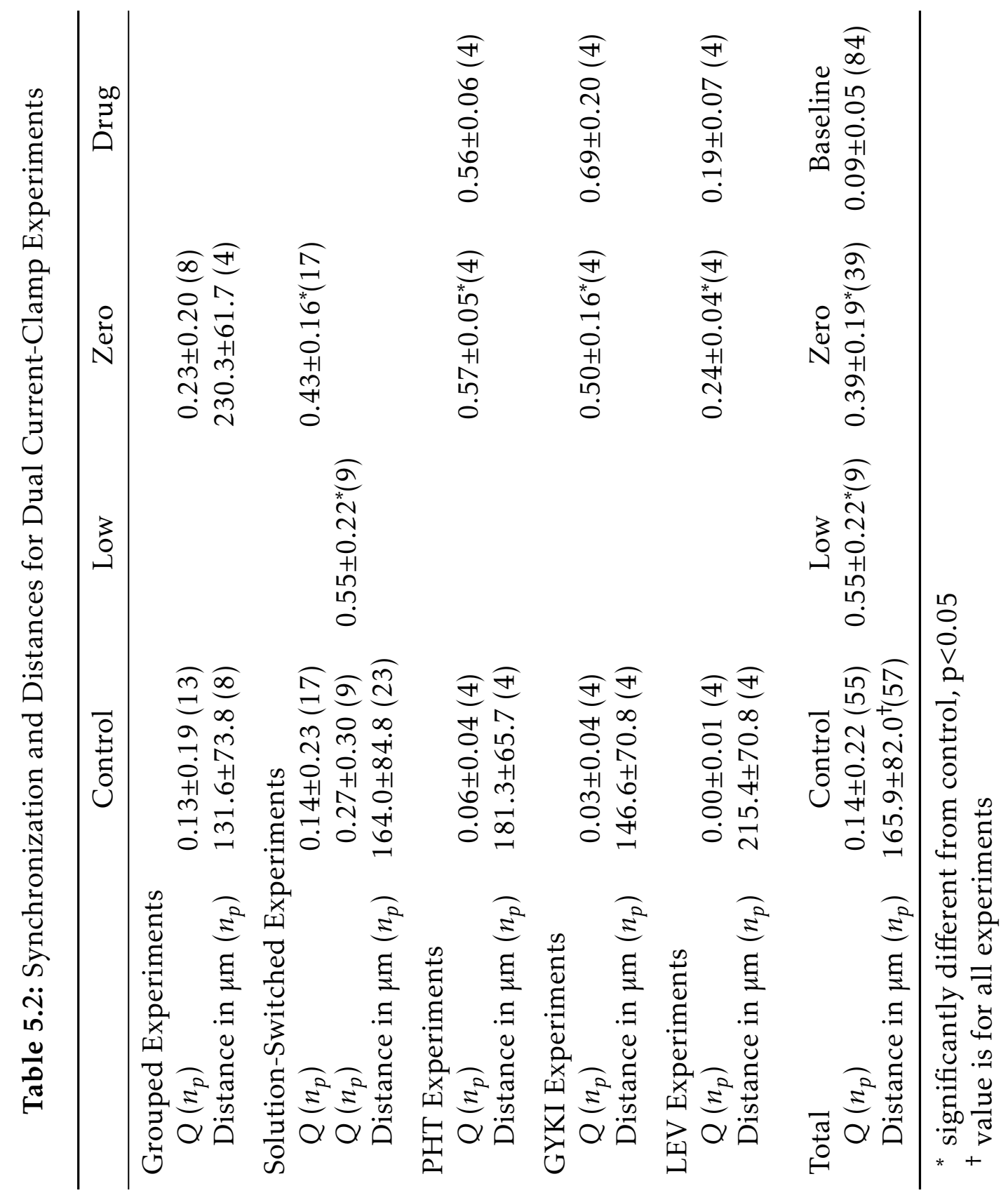


robustness against noise [153]. ES was not affected by the increase of frequency during bursting activity, which was verified using surrogate data. Additionally, action potentials may not exhibit exact synchronization $[38,130]$ or even a preferred order between cells as demonstrated by the small $|q|$ values. By using ES, slight variations in delay and order could be considered synchronous within a small time window.

Synchronization of cells with biological synaptic connections has been studies using neuronal models, and the type of connection often affects the ability of the cells to in-phase synchronize $[89,133]$. However, in these experiments the type of connection did not alter the synchronization of cells in CM. But high values of synchronization did appear occasionally in $\mathrm{CM}$, suggesting that it is possible for biologically connected cells to be highly synchronized, but it was not common under the conditions studied. Modeling of bursting activity suggest that synchronization occurs on the level of bursts but not at the level of action potentials $[130,154]$ unless the synaptic strength is sufficiently strong [155]. Results in solution-switch experiments confirm that the action potential synchronization was increased for decreased $\mathrm{Mg}^{2+}$ concentrations.

Performing quantal analysis under decreased $\mathrm{Mg}^{2+}$ was difficult due to the increase of activity and superposition of multiple quantal releases. Because of these issues, quantal analysis was measured in two ways: method of failures and deconvolution of amplitude histograms. Both methods suggest that more vesicles were released per EPSC, indicating a strengthening of connections. The first peaks from amplitude distributions were all around $20 \mathrm{pA}$ suggesting that the quantal content of one vesicle was unaltered by decreasing $\mathrm{Mg}^{2+}$ as has been described before [135]. Also, decreasing $\mathrm{Mg}^{2+}$ increased the number and sizes of sEPSCs. New populations emerged at LM and ZM conditions suggesting that the strength of the 
synapses increased.

The strength of connectivity was increased as $\mathrm{Mg}^{2+}$ was decreased, but the synchronization was higher in LM conditions, which suggest that the $\mathrm{Mg}^{2+}$ concentration may have a dose response with a maximum effectiveness (at synchronizing) around $0.5 \mathrm{mM}$. Decreasing the $\mathrm{Mg}^{2+}$ concentration to $0.5 \mathrm{mM}$ may have the optimal effect on opening channels and facilitating release of neurotransmitter, while further decrease in $\mathrm{Mg}^{2+}$ concentration leads to saturation of receptors and/or depletion of the ready releasable pool.

PHT, which stops recurrent action potentials by blocking $\mathrm{Na}^{+}$channels $[144$, 145], did not alter synchronization as expected. GYKI blocks AMPA and kainate receptors [146], which play a role in the bursting behavior of ZM [135], but this blockade seemed to increase the synchronization, though not significantly. Golomb et al. [130] demonstrated in a neuron model with reduced extracellular $\mathrm{Mg}^{2+}$ that decreasing AMPA conductance transitions from a bursting state to a tonic state or tristable state (quiescent, bursting or tonic behavior). This may explain why addition of GYKI attenuated the bursting activity. The increase in synchronization with GYKI could be similar to the result seen with LM conditions, where the synaptic strength was not as strong as in the ZM condition but the synchronization was higher.

LEV has multiple mechanisms of action [147-149], and has been shown in some in vitro models to decrease synchronization [33]. In these experiments, LEV decreased the synchronization of the cells, although not significantly, and very slightly altered the bursting pattern without stopping bursting. Some cells in GYKI and LEV did not have recurrent action potentials in CM conditions. In ZM conditions all cells fired action potentials on their own, but the cells switched to ZM + LEV had a decreased level of synchronization in ZM as compared to the other drug 
experiments. This may be a reason that LEV did not show a significant decrease in synchronization. More experiments are needed to confirm a significant change in synchronization.

These results have some limitations. Synaptic connections in cells are dynamic and can display short-term effects such as depression, facilitation and plasticity [156]. These properties were not accounted for in the analysis. Only three concentrations of $\mathrm{Mg}^{2+}$ were examined in this chapter. The maximum synchronization was found in LM conditions. More experiments at various concentrations of $\mathrm{Mg}^{2+}$ are needed to verify that there is a dose response and to find the concentration of maximal synchronization.

\subsection{Conclusions}

These findings suggest that increase in synaptic strength also caused an increase in action potential synchronization for pairs of neurons in culture. The addition of three antiepileptic drugs, while significantly altering the firing distribution of the cells, did not significantly affect the synchronization of two cells as compared to no drug added. Addition of PHT, which does not affect the strength of synaptic connections, had no effect on the synchronization of action potentials. GYKI affects synaptic connections by blocking AMPA and kinate receptors but it caused a slight increase in synchronization. Addition of LEV slightly decreased the synchronization, but the synchronization measured in ZM was lower than other groups of cell pairs. More experiments are needed to know if the changes seen in GYKI and LEV synchronization are significant. 


\section{Chapter 6}

\section{Conclusions and Future Work}

\subsection{Conclusions}

This dissertation examines the role of synchronization of neural systems, specifically synchronization in epilepsy. Synchronization is examined on the level of the brain and on the level of the individual neuron.

The synchronization index was calculated from intercranial EEGs of patients with medically intractable mesial temporal lobe epilepsy. Five distinct stages were identified that were similar across all patients and all seizures. Patients that became seizure free after resection had a lower SI value during the seizure hub than those that were only seizure free while on medication. It is possible that SI can be used as a tool to determine surgical outcomes and underlying network dynamics during seizures.

A phase model was built for a hippocampal neuron in culture. Two isolated neurons were then subjected to linear feedback at specific delays to create an in-phase and anti-phase state based on the phase model. The phase model was also used predict the behavior of a large group of neurons under linear feedback. 
Desynchronization would be a desirable state to engineer, since synchronization plays a role in the initiation and spread of seizures. A range of feedback delays were found that would desynchronize the population.

Neurons in culture can also synchronize through synaptic connections. Synchronization between two cells can be strengthened by altering the external medium. Decreasing the $\mathrm{Mg}^{2+}$ concentration was shown to increase the strength of connection between cells, but the overall synchronization had a possible dose response. Addition of three typical AEDs did not significantly alter the synchronization created by zero $\mathrm{Mg}^{2+}$, but they showed three different effects. Additional experiments are needed to verify the results of the AEDs.

\subsection{Future Work}

\subsubsection{EEG Analysis Based on SI Calculations}

The methods used in Chapter 3 will be extended to more patients and seizures to examine clinical relevance and consistency. This work will be further expanded by using network analysis to quantify and describe the changes in the SI connectivity seen in the animations and stages put forth in Section 3.4.2. These network measures can describe differences in the topology of the whole network and changes at an electrode. Similar network analyses have been done for recorded seizures using cross-correlation to create a connection matrix [157].

The programs describe in Section 3.3.2 have already been adapted to allow filtering of the EEG into physiologically relevant bands for analysis. All of the methods that have been described can be repeated for each frequency band. Additionally, the programs saves the independent components of SI and future analysis could focus on only the phase synchronization between the EEG signals or amplitude of 
the EEG if needed.

\subsubsection{Extension of Phase Model and Feedback Design}

Chapter 4 demonstrated the ability of a feedback signal to desynchronize neurons for an idealized system. In the ideal conditions, the feedback signal can be designed to create any desired synchronization states. Future experiments are needed to extend the results from the ideal system to design a feedback for treatment of epilepsy or other diseases. The first difference from the ideal system to the real system is the presence of synaptic connections. Generally, the feedback signal can be made strong enough to overcome these intrinsic interactions, but it would also be beneficial to have a model for each of the synaptic connection types. Also, the experiments in Chapter 4 allowed precise control and measurement of the individual neuron membrane potentials. In whole animal and human experiments, feedback signals would be applied with an external electrode and individual neurons would receive slightly different stimulation based on their distance from the electrode. Finally, the activity of individual neurons were modified in Chapter 4 to be periodic. Actual individual neuron behavior during seizures are most likely different and may modify the feedback parameters needed to desynchronize the system. Below are suggested experiments to address some of these difference between the ideal and real systems.

\section{Dual Whole-Cell Patch-Clamp Experiments}

Neuron micro-islands could be cultured [116] and the interaction function could be measured for two naturally coupled neurons [114]. The system would not require feedback, but rather the natural oscillation and interaction of the two neurons would provide the data to measure the interaction function. This method would 
allow the measurement of the contribution of the individual synapses by varying bicuculline, DNQX and DL-AP5 concentrations in the external medium, blocking some synapses but not all. Assuming that the contributions to the interaction function sum linearly, the different interactions functions, with and without the synapses, would just be subtracted.

\section{Extracellular Stimulation Experiments}

It is impossible to make similar measurements to whole-cell patch clamp recordings in a whole animal or a human brain. To extend the work in Chapter 4, experiments could be conducted in which two cells are whole-cell patch-clamped in culture and extracellular electrodes are used to record and stimulate the network of neurons. Bursting activity of the neurons could be generated by the addition of bicuculline, 4-aminopyridine or decreased external magnesium. The field potential recorded by the extracellular electrode could be used to generate a feedback signal, which would then be applied by a different extracellular bipolar electrode [158]. The dual whole cell recording would demonstrate how the potential of the neurons are affected either nearby or at a distance from a stimulation electrode. Combining dual whole-cell recordings to an extracellular electrode recording may demonstrate how the underlying neuron activity contributes to the mean field recording $[28,30]$.

\section{Whole Animal Experiments}

After it was demonstrated that linear feedback can affect the phase synchronization of two neurons (see Chapter 4), Xin Ren began independent experiments on how feedback affects the dynamics of seizures [159]. Current research showed that closed-loop stimulation using linear feedback at the focus of chemoconvulsively initiated seizures in rats reduces the amplitude of the seizure [160]. However, how 
the feedback affects the behavior of the neurons is unknown. Studies have been designed to determine how different types of feedback affect individual neurons in anestitized and awake rat models using microelectrode arrays. The goal is to develop an understanding of neuron activity during a seizure and then design a feedback signal that can effectively stop a seizure.

An apparatus with the capability of recording eight signals, calculating a feedback signal and applying current at $25 \mathrm{kHz}$ was built, similar to the neuron apparatus. Rats were electrically kindled to create seizures. One bipolar electrode was placed in the hippocampus CA3 to initiate the seizures, one microelectrode array with four points of contact was placed in contralateral CA1 of the hippocampus to record the seizure activity. Initial studies have demonstrated in anesthetized and awake animals that synchronization is high at the beginning and middle of the seizure, with a decrease in synchronization towards the end of the seizure [159].

Future studies will examine the role of synchronization between multiple areas of the hippcampus and entorhinal cortex. One bipolar electrode will be placed in the hippocampus CA3 to initiate the seizures, as before. Two microelectrode arrays with four contacts each will be used to record activity from two of the following areas: hippocampus CA1, dentate gyrus and entorhnial cortex. After examining the synchronization across these areas for seizure without treatment, the effect of anticonvulsants and electrical stimulation on the synchronization of these areas can be measured and compared to find effective treatments. A variety of stimulations can be explored, such as sine waves, pulses, linear and non-linear feedback.

\subsubsection{Synaptic Strength and Synchronization}

Experiments are planned to continue the work in Chapter 5. More dual patch recordings will be gathered to further examine the effect of AEDs on the synchro- 
nization of neural action potentials in culture. Experiments will follow the same methods as described in Section 5.3.2.

Additionally, the same analysis in Section 5.3.3 could be extended to examine the effect of a variety of parameters in the cultures:

1. Bursting activity created by other in vitro epilepsy models such as bicuculline, 4-aminopyridine or high $\mathrm{K}^{+}$. Different mechanisms underly the bursting activity and may alter the strength of the synchronization.

2. Distance of the pair in culture. Distance would affect the direct connections between the two neurons, but could also affect the inputs each neuron receives. If the cells surrounding the neurons are different, their inputs are more likely to be different, possibly resulting in a lower level of synchronization during bursting.

3. Synchronization of sEPSCs. By blocking action potentials in both cells with lidocane in the internal solutions, two cells could be patch clamped in voltageclamp mode and the synchronization of the sEPSCs could be examined as a function of $\mathrm{Mg}^{2+}$. This experiment would demonstrate how the inputs to each cells are synchronized as the strength of the network is increased, rather than the synchronization of the action potentials of the two cells within the network.

4. Examination of additional $\mathrm{Mg}^{2+}$ concentrations and the alteration of $\mathrm{Ca}^{2+}$. $\mathrm{A}$ more detailed study could be done of the relationship between the synaptic strength and synchronization of action potentials in culture. Smaller $\mathrm{Mg}^{2+}$ concentration changes would allow the correlation between increase of synaptic strength and synchronization without bursting activity. Under bursting 
conditions, more $\mathrm{Mg}^{2+}$ concentrations would verify the dose-response relationship where the synchronization seems to be maximum at a concentration of $0.5 \mathrm{mM} \mathrm{Mg}^{2+}$, even though the strength of the synaptic connections are higher in $0 \mathrm{mM} \mathrm{Mg}{ }^{2+}$. Increasing $\mathrm{Ca}^{2+}$ would also increase synaptic connections by facilitating synaptic vesicle release to see if the synchronization stays constant or decreases for further increases in synaptic strength.

The Neuro-Synchroscope 2008 software and apparatus set up in Fig 5.1 do not need to be modified to perform these experiments. 


\section{Appendix A}

\section{MATLAB Code for Human EEG Data}

\section{Analysis}

\section{A.1 Calculate SI from EDF files}

\section{A.1.1 SI_file_create}

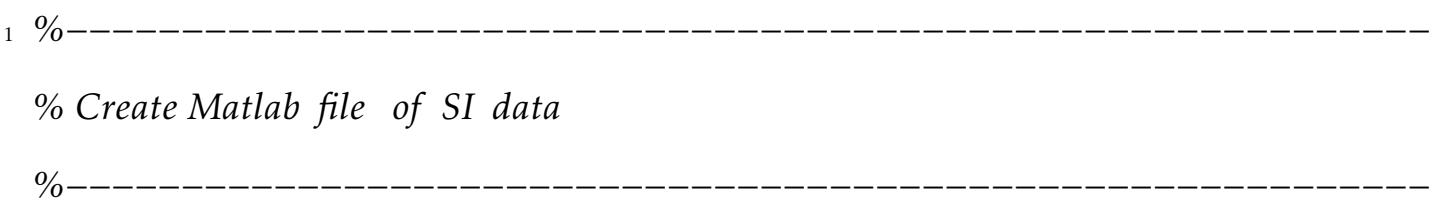

function SI_file_create_newformat_May_2011(edf_file_name,matrix_of_electrodes, samp_freq,output_name,filter_switch,filter_band)

6

\% loads raw data

[raw_data,header $]=$ sload(edf_file_name);

$\%$ removes only electrodes to be analyzed

cut_data=raw_data(:,matrix_of_electrodes); 
$11 \%$ creates a table of time stamps

time_table $=(1:$ length $($ raw_data $)) /$ samp_freq;

$\%$ loads electrode names

electrode_names = header.Label(matrix_of_electrodes,:);

$\%$ finds number of electrodes

16 number_of_electrodes = length(matrix_of_electrodes);

$\%$ finds number of sigmas to go with SI's

number_of_sigmas $=($ length $($ raw_data $) /$ samp_freq $)$;

$\%$ Electrode pair generator

21 counter $=1$;

for $\mathrm{i}=1$ :number_of_electrodes-1

for $\mathrm{j}=\mathrm{i}+1$ :number_of_electrodes

electrodepair $($ counter, 1$)=\mathrm{i}$;

electrodepair $($ counter, 2$)=j$;

$26 \quad$ counter $=$ counter +1 ;

end

end

\% FILTER, uses cheby2_filter_func, but can use any other filter if filter_switch is 1, or turned 'on', the filter band range must be defined

31 if filter_switch $==1$

fs = samp_freq;

hzrange $=$ filter_band;

data $=$ cut_data;

$\%$ extracted data is now filtered

$36 \quad[$ data, cut_data $]=\underline{\text { cheby2_filter_func }}($ fs , hzrange,data); 
end

$\%$ hilbert of the extracted data

hilbert_data =hilbert $($ cut_data);

41 amplitude=abs(hilbert_data);

hilbert_unwrap=unwrap(angle(hilbert_data));

$\%$ finds size of the electrodepairs that need to be calculated

[num_elec,byy $]=$ size(electrodepair);

$46 \%$ time stamp loop

for $\mathrm{m}=\mathrm{sn}$ :number_of_sigmas

$\%$ electrode loop

for $n=1:$ num_elec

elec1 = electrodepair $(\mathrm{n}, 1)$;

51

elec2 = electrodepair $(\mathrm{n}, 2)$;

$\%$ finds SI for two electrodes at a certain time stamp

[SI,Sigma_pair,amp_elec1,amp_elec2] = partsplot5 (cut_data,time_table,amplitude,

hilbert_unwrap,m,number_of_sigmas,elec1,elec2);

$\%$ sets SI/sigma into matrix of dimension (Time, electrode 1, electrode 2)

SI_matrix $(\mathrm{m}$, elec1,elec2 $)=$ SI;

56

SI_matrix $(\mathrm{m}$, elec $2, \mathrm{elec} 1)=\mathrm{SI}$;

sigma_matrix $(\mathrm{m}, \mathrm{elec} 1, \mathrm{elec} 2)$ = Sigma $\_$pair;

sigma_matrix $(\mathrm{m}, \mathrm{elec} 2$,elec1 $)$ = Sigma $\_$pair;

$\%$ sets amp for each electrode;

$\operatorname{amp} \_\operatorname{matrix}(\mathrm{m}, \mathrm{elec} 1)=$ amp_elec 1 ;

61 $\operatorname{amp} \_\operatorname{matrix}(\mathrm{m}, \mathrm{elec} 2)=$ amp_elec 2 ;

end 


\section{$\operatorname{disp}($ strcat ('Time stamp:', num2str(m)));}

end

${ }_{66} \%$ saves generated SI Matrix

save(output_name,'SI_matrix','electrode_names','sigma_matrix', 'amp_matrix');

$\operatorname{disp}('$. mat file saved');

\section{A.1.2 cheby2 filter func}

1 function $[$ data, signal_extract $]=\underline{\text { cheby } 2 \text { _filter_func }}(\mathrm{fs}$, hzrange,data $)$

$\% \%$ Input

[ size_length, maxelect $]=\operatorname{size}($ data $)$;

signal_extract $=$ zeros(size_length, maxelect);

6

for electrode $=1$ :maxelect

$\% \%$ Create Filter

$\%$ nyquist freq

HalfFs = fs $/ 2$;

$11 \%$ Tukey Window to set ends to zero + enhance filter

windowed=tukeywin(size_length,0.001);

$\mathrm{x}(:$, electrode $)=$ data $(:$, electrode $) *$ windowed;

\% Stop-band test removes $60 \mathrm{hz}$ Power noise (from 55 to $65 \mathrm{hz}$ )

16 fstop $=[(55) /(\mathrm{fs} / 2),(65) /(\mathrm{fs} / 2)]$;

$[\mathrm{b}, \mathrm{a}]=\operatorname{butter}(10$, fstop, 'stop' $)$;

hz60pass $=$ filtfilt $(\mathrm{b}, \mathrm{a}, \mathrm{x}(:$, electrode $))$; 
$\%$ Cheby2 Filter with optimized $n$ and $R$

$21 \quad \mathrm{n}=4$;

$\mathrm{R}=20$;

Wst = hzrange/HalfFs;

$[\mathrm{b} 1, \mathrm{a} 1]=$ cheby $2(\mathrm{n}, \mathrm{R}, \mathrm{Wst})$;

signal_extract $(:$, electrode $)=$ filtfilt $(\mathrm{b} 1, \mathrm{a} 1, \mathrm{hz60}$ pass);

$26 \operatorname{disp}(\operatorname{strcat}($ 'electrode:' , num2str(electrode)));

end

$\operatorname{disp}('$ Filter complete');

\section{A.1.3 partsplot5}

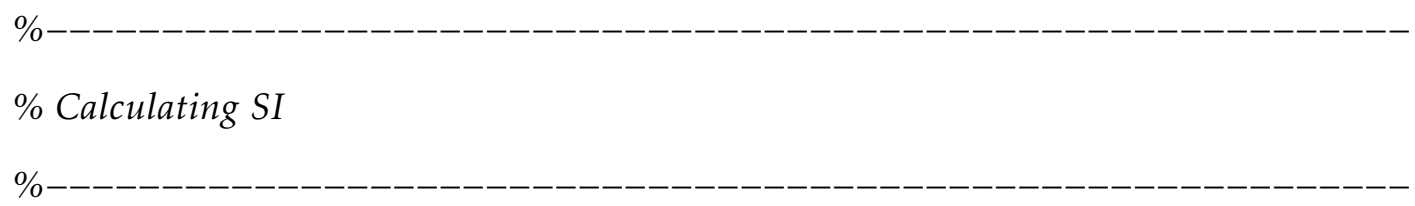

4

function [SI,Sigma_pair,amp_elec1,amp_elec2]=partsplot5 (data,time_table,amplitude, hilbert_unwrap,time_tick,number_of_sigmas,elec1,elec2)

Ndata $=$ round $($ length $($ data $) /$ number_of_sigmas -0.5$)$;

$\mathrm{dp}=$ hilbert_unwrap(:,elec1)-hilbert_unwrap(:,elec2);

$9 \operatorname{tm}($ time_tick $)=$ mean $($ time_table $($ Ndata $*($ time_tick -1$)+1:$ Ndata $*$ time_tick $))$;

$\operatorname{am}($ time_tick,elec 1$)=$ mean $(\operatorname{amplitude}(\operatorname{Ndata} *($ time_tick -1$)+1:$ Ndata $*$ time_tick,elec 1$))$; $\operatorname{am}($ time_tick,elec 2$)=$ mean $\left(\right.$ amplitude $\left(\right.$ Ndata $*($ time_tick -1$\left.\left.)+1: N d a t a * t i m e \_t i c k, e l e c 2\right)\right)$; $\operatorname{sigma}($ time_tick,elec 2$)=$ getsigma $(\operatorname{dp}($ Ndata $*($ time_tick -1$)+1:$ Ndata $*$ time_tick $))$; SI $=(($ am $($ time_tick,elec1 $)+a m($ time_tick,elec 2$)) / 2) . *$ sigma $($ time_tick,elec 2$)$;

${ }_{14}$ Sigma_pair = sigma(time_tick,elec2); 
amp_elec1 = am(time_tick,elec1);

amp_elec2 = am(time_tick,elec2);

\section{A.1.4 getsigma}

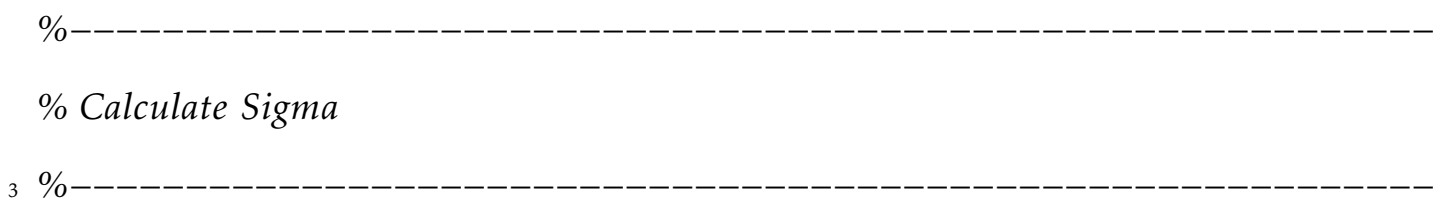

function $\mathrm{O}=$ getsigma $(\mathrm{dp})$

$\mathrm{dp}=\bmod (\mathrm{dp}, 2 \times \mathbf{p i})$;

8 bin number $=30$;

number_bins $=$ linspace $(0,2 * \mathbf{p i}$,bin_number $)$;

$[\mathrm{ny}, \mathrm{nx}]=$ hist(dp,number_bins);

$\mathrm{pk}=\mathrm{ny} / \operatorname{sum}(\mathrm{ny})$;

ind $=$ find $(\mathrm{pk}==0)$;

$13 \mathrm{pk}($ ind $)=[]$;

$\operatorname{Smax}=\log (30)$;

$\mathrm{S}=-1 * \operatorname{sum}(\mathrm{pk} . \star \log (\mathrm{pk}))$;

$\mathrm{O}=1-\mathrm{S} / \mathrm{Smax}$;

\section{A.1.5 analyze_quick}

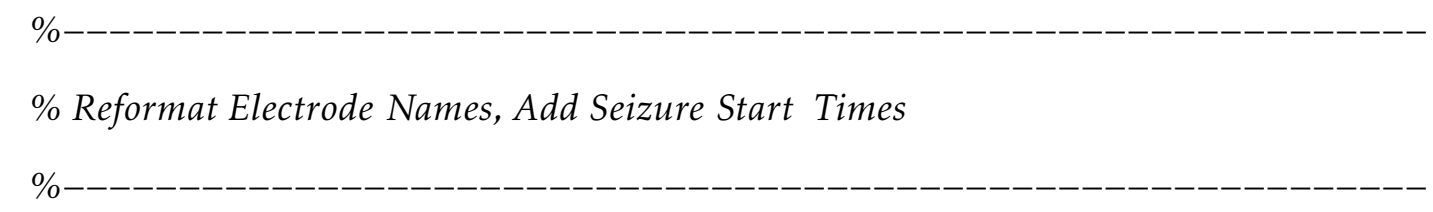

4

function analyze_quick(mat_file_name,seizure_start_time,output_name,output_folder) 
load(mat_file_name); \% Load the file

[rows,columns,pages]=size(SI_matrix); \% Find the size of the time matrix

9

$\%$ Create a cell array of the electrode names without the -REF

electrode_name = cellstr(electrode_names);

$\mathrm{x}=\operatorname{regexp}($ electrode_name,'-');

$\mathrm{x}=\operatorname{cell} 2 \mathrm{mat}(\mathrm{x})$;

14 for $i=1$ :pages

electrode_name $\{i, 1\}=$ electrode_name $\{\mathrm{i}, 1\}(1:(\mathrm{x}(\mathrm{i})-1))$;

end

$\%$ Create a filename for the analyzed data

19 end_name = strfind (output_name,'.mat');

filename = output_name;

filename1 = strcat(output_folder, 'raw_,, filename(1:end_name-1),'.mat');

save(filename1, 'electrode_name','SI_matrix' , ' seizure_start_time' , 'amp_matrix', ' sigma_matrix')

end

\section{A.2 Create SI Movies and Still Images}

\section{A.2.1 make_image}

$2 \%$ Make Still Image of SI Pairs

$\%$ 
function [file_type ] = $\underline{\text { make_image }}($ electrode_matrix,electrode_name,data,cmin,cmax, image_mode)

$7 \% \%$ Load and plot the brain image, load the electrodes

[image_data] = imread('brain_plain',' tif ') ;

image(image_data $(:,:, 1: 3))$

hold on

$\%$ Generic placement of all possible electrodes

12 load electrode_map

$\operatorname{set}(\mathbf{g c f}$, ' color' , 'white')

$\% \%$ Find the non-used electrodes and remove

\% String compare for the second column (list of patient electrodes in excel file)

17 [blank_electrodes] = find $(\operatorname{strcmp}(' b l a n k '$, electrode_matrix $(:, 2))==1)$;

$\%$ Delete the rows that have blank electrodes, both in location and name

electrode_matrix(blank_electrodes,$:)=[]$;

electrodeXY(blank_electrodes,: $)=[]$;

${ }_{22} \% \%$ Create image based on type specified by image_mode

if isempty(image_mode) $==1 \%$ Normal movie mode, where the max SI for each electrode is plotted

$\% \%$ Plot the electrodes that are active, with connections

$\%$ Plotting the maximum SI connection for every electrode

[number_of_rows,ry] = size(data); \% number_rows and number_columns should be equal and equal number of electrodes

$27 \%$ Preallocating for speed, one column, length of the number of electrodes max_SI = zeros(number_of_rows,1); 
$\%$ Index the color for each electrode and line

$\mathrm{h}=$ figure $(1)$;

colormap_scale $=255$;

32

Color $=$ jet $($ colormap_scale);

colormap_index $=\mathbf{f i x}(($ data $-\mathrm{cmin}) /(\operatorname{cmax}-\mathrm{cmin}) *($ colormap_scale -1$))+1$;

$\%$ Set all values off the range to the highest color

$[\mathrm{i}, \mathrm{j}, \mathrm{k}]=$ find $($ colormap_index $>255)$;

for counter $=1$ :length $(i)$

colormap_index $(\mathrm{i}($ counter $), \mathrm{j}($ counter $))=255$;

end

for electrode_1 = 1:number_of_rows \% Increment through every electrode $\%$ Find and index maximum SI for this electrode

$\left[\right.$ ry, electrode $\left.\_2\right]=\operatorname{find}\left(\operatorname{data}_{(}\left(\right.\right.$electrode $\left._{-} 1,:\right)==\max \left(\right.$ data $_{2}\left(\right.$ electrode $\left.\left.\left._{-} 1,:\right)\right)\right)$;

$\%$ Keep the maximum SI in a matrix

$\max \_S I\left(\right.$ electrode $\left.\_1\right)=\max \left(\right.$ data $\left(\right.$ electrode $\left.\left.\_1,:\right)\right)$;

$\%$ Get the name of the electrodes, match the name of the electrodes to the electrode position on the image

electrode_1_position $=$ find $(\operatorname{strcmp}($ electrode_name $($ electrode_1 $)$, electrode_matrix $(:, 2))==1)$;

electrode_2_position $=$ find $\left(\operatorname{strcmp}\left(\right.\right.$ electrode $\_n a m e\left(e l e c t r o d e \_2\right)$, electrode_matrix $(:, 2))==1)$; $\%$ Plots one electrode for the pair, with SI mag in color $\operatorname{plot}($ electrodeXY(electrode_1_position,1),electrodeXY(electrode_1_position,2), 'o' ,'MarkerFaceColor',Color(colormap_index(electrode_1,electrode_2),:),' MarkerEdgeColor',Color(colormap_index(electrode_1,electrode_2),:)) 
$\%$ Plots the line between them, with SI mag in color

line ([electrodeXY(electrode_1_position,1) electrodeXY(electrode_2_position,1)], [electrodeXY(electrode_1_position,2) electrodeXY(electrode_2_position,2)],' Color',Color(colormap_index(electrode_1,electrode_2),:))

end

axis off

$\operatorname{colormap}($ jet $(255))$

colorbar('YTick',[50:50:250], 'YTickLabel', \{num2str(ceil(50*10/255*(cmax-cmin)) $/ 10+\operatorname{ceil}(\operatorname{cmin} * 10) / 10), \operatorname{num} 2 \operatorname{str}(\operatorname{ceil}(100 * 10 / 255 *(\mathrm{cmax}-\mathrm{cmin})) / 10+\operatorname{ceil}(\mathrm{cmin}$ $\star 10) / 10), \operatorname{num} 2 \operatorname{str}(\operatorname{ceil}(150 * 10 / 255 *(\operatorname{cmax}-\operatorname{cmin})) / 10+\operatorname{ceil}(\operatorname{cmin} * 10) / 10)$, $\operatorname{num} 2 \operatorname{str}(\operatorname{ceil}(200 * 10 / 255 *(\operatorname{cmax}-\operatorname{cmin})) / 10+\operatorname{ceil}(\mathrm{cmin} * 10) / 10), \operatorname{strcat}('>$ ', $\operatorname{num} 2 \operatorname{str}(\operatorname{ceil}(250 * 10 / 255 *(\operatorname{cmax}-\operatorname{cmin})) / 10+\operatorname{ceil}(\operatorname{cmin} * 10) / 10))\}) ;$ file_type $=1$;

elseif isnumeric(image_mode) $==1 \%$ image_mode contains the number of top electrodes to pick

$\%$ Plotting the top \#\# of maximum SI connections $[$ reshape_data $]=$ reshape $($ data,length $($ data $) \times$ length $($ data $), 1) ; \%$ Make a long vector of all SI values

[ry,electrode_index,rx] = unique(reshape_data); \%Find non-repeating SI values ( sorted ascending values)

$\left[\mathrm{NaN}\right.$ index, $\left.{ }^{\sim}\right]=\operatorname{find}\left(\operatorname{isnan}(\mathrm{ry})==1,1\right.$, 'first' $\left.^{\prime}\right)$; \% Find NaNs, start SI plots after NaNs

[electrode_1, electrode_2] = ind2sub([length(data) length(data)],electrode_index( NaN_index-1-image_mode+1:NaN_index-1)); \%Find the electrode pairs that correspond to the top SI values 
$\%$ Index the color for each electrode and line

67 $\mathrm{h}=$ figure $(1)$;

colormap_scale $=255$;

Color $=$ jet $($ colormap_scale);

colormap_index $=\mathbf{f i x}(($ data $-\mathrm{cmin}) /(\operatorname{cmax}-\mathrm{cmin}) *($ colormap_scale -1$))+1$;

$72 \%$ Set all values off the range to the highest color

$[\mathrm{i}, \mathrm{j}, \mathrm{k}]=$ find $($ colormap index $>255)$;

for counter $=1$ :length $(i)$

colormap_index $(\mathrm{i}($ counter $), \mathrm{j}($ counter $))=255$;

end

77

$\%$ Plot the electrodes that are active, with connections

for index_electrode $=1$ :image_mode

$\%$ Get the name of the electrodes, match the name of the electrodes to the electrode position on the image

electrode_1_position $=$ find $(\operatorname{str} \mathbf{c m p}($ electrode_name(electrode_1(index_electrode) ),electrode_matrix $(:, 2))==1)$;

electrode_2_position $=$ find $(\mathbf{s t r} \mathbf{c m p}$ (electrode_name(electrode_2(index_electrode) ),electrode_matrix $(:, 2))==1)$;

$\%$ Plots one electrode for the pair, with SI mag in color

plot(electrodeXY(electrode_1_position,1),electrodeXY(electrode_1_position,2), 'o' ,'MarkerFaceColor',Color(colormap_index(electrode_1(index_electrode), electrode_2(index_electrode)),:),'MarkerEdgeColor',Color(colormap_index( electrode_1(index_electrode),electrode_2(index_electrode) $),:))$

$\%$ Plots the second electrode for the pair, with SI mag in color plot(electrodeXY(electrode_2_position,1),electrodeXY(electrode_2_position,2), 'o' 
,'MarkerFaceColor',Color(colormap_index(electrode_1(index_electrode), electrode_2(index_electrode)),:),'MarkerEdgeColor',Color(colormap_index( electrode_1(index_electrode),electrode_2(index_electrode)),:))

$\%$ Plot the electrodes that are not active active_electrodes $=[$ electrode_1; electrode_2 $]$;

[electrodes_plotted,rx ,ry]=unique(active_electrodes);

for electrode_counter $=1:$ length $($ data $)$

[i] = find(electrode_counter $==$ electrodes_plotted); \%See if the electrode is active

if isempty $(\mathrm{i})==1 \%$ electrode of electrode_counter is not active, so plot $\%$ Get the name of the electrodes, match the name of the electrodes to the electrode position on the image

electrode_position = find $(\mathbf{s t r c m p}$ (electrode_name(electrode_counter), electrode $\_$matrix $\left.\left.(:, 2)\right)==1\right)$;

$\%$ Plots the electrode in black plot(electrodeXY(electrode_position,1),electrodeXY(electrode_position,2), 'o' ,' 'MarkerFaceColor', 'k','MarkerEdgeColor', 'k')

else \% electrode of electrode_counter is already plotted $\%$ Do nothing

end 
end

axis off

107

$\operatorname{colormap}($ jet $(255))$

colorbar('YTick',[50:50:250], 'YTickLabel', \{num2str(ceil $(50 * 10 / 255 *(\operatorname{cmax}-\mathrm{cmin}))$

$/ 10+\operatorname{ceil}(\operatorname{cmin} * 10) / 10), \operatorname{num} 2 \operatorname{str}(\operatorname{ceil}(100 * 10 / 255 *(\operatorname{cmax}-\mathrm{cmin})) / 10+\operatorname{ceil}(\mathrm{cmin}$

$\star 10) / 10), \operatorname{num} 2 \operatorname{str}(\operatorname{ceil}(150 * 10 / 255 *(\operatorname{cmax}-\operatorname{cmin})) / 10+\operatorname{ceil}(\operatorname{cmin} * 10) / 10)$,

$\operatorname{num} 2 \operatorname{str}(\operatorname{ceil}(200 * 10 / 255 *(\operatorname{cmax}-\operatorname{cmin})) / 10+\operatorname{ceil}(\operatorname{cmin} * 10) / 10), \operatorname{strcat}('>$ ',

num $2 \operatorname{str}(\operatorname{ceil}(250 * 10 / 255 *(\operatorname{cmax}-\operatorname{cmin})) / 10+\operatorname{ceil}(\operatorname{cmin} * 10) / 10))\})$;

file_type $=2$;

else \% movie_mode contains a string with the electrode to plot a number of top SIs for $\%$ Plotting the top \#\# of maximum SI for a specific electrode (max: \# electrodes - 1)

$\%$ Get the column of data that corresponsds to the desired electrode

electrode_1 = find $(\operatorname{strcmp}($ image_mode $\{1\}$,electrode_name $)==1)$;

$\%$ Get position of electrode 1

electrode $\_1 \_$position $=$find $\left(\operatorname{strcmp}\left(\right.\right.$ electrode_name $\left(\right.$ electrode $\left.\_1\right)$,electrode $\_$matrix $(:, 2))==1)$;

$117 \%$ Sort the data in descending order for that electrode column

$[$ ry, electrode_2a $]=\operatorname{sort}\left(\right.$ data $\left(:\right.$, electrode $\left.\_1\right)$,'descend' $)$;

$\%$ Limit electrode_2 to the top number of electrodes

electrode $\_2=$ electrode $\_2 a\left(2:\right.$ image $\_$mode $\left.\{2\}+1\right)$;

$\%$ Index the color for each electrode and line

$122 \mathrm{~h}=$ figure $(1)$;

colormap_scale $=255$;

Color $=$ jet $($ colormap_scale $)$; 
colormap_index $=\mathbf{f i x}(($ data $-\mathrm{cmin}) /(\operatorname{cmax}-\mathrm{cmin}) *($ colormap_scale -1$))+1$;

127

132

$\%$ Set all values off the range to the highest color

$[\mathrm{i}, \mathrm{j}, \mathrm{k}]=$ find $($ colormap_index $>255)$;

for counter $=1:$ length $(\mathrm{i})$

colormap_index $(\mathrm{i}($ counter $), \mathrm{j}($ counter $))=255$;

end

$\%$ Plot the electrodes that are active, with connections

for index_electrode $=$ image_mode $\{2\}:-1: 1$

$\%$ Get the name of the electrodes, match the name of the electrodes to the electrode position on the image

electrode_2_position $=$ find $(\mathbf{s t r} \mathbf{c m p}$ (electrode_name(electrode_2(index_electrode) ),electrode_matrix $(:, 2))==1)$;

$\%$ Plots one electrode for the pair, with SI mag in color

plot(electrodeXY(electrode_1_position,1),electrodeXY(electrode_1_position,2), 'o'

,'MarkerFaceColor',Color(colormap_index(electrode_1,electrode_2(

index_electrode)),:),'MarkerEdgeColor',Color(colormap_index(electrode_1, electrode_2(index_electrode)),:))

$\%$ Plots the second electrode for the pair, with SI mag in color

plot(electrodeXY(electrode_2_position,1),electrodeXY(electrode_2_position,2) , o'

,'MarkerFaceColor',Color(colormap_index(electrode_1,electrode_2(

index_electrode)),:),'MarkerEdgeColor',Color(colormap_index(electrode_1, electrode_2(index_electrode)),:))

$\%$ Plots the line between them, with SI mag in color

line ([electrodeXY(electrode_1_position,1) electrodeXY(electrode_2_position,1)], [electrodeXY(electrode_1_position,2) electrodeXY(electrode_2_position,2)],' 
Color',Color(colormap_index(electrode_1,electrode_2(index_electrode) $),:$ ) )

end

$\%$ Plot the electrodes that are not active

active_electrodes $=$ [electrode_1; electrode_2 $]$;

147

[electrodes_plotted ,ry,rx]=unique(active_electrodes);

for electrode_counter $=1$ :length $($ data $)$

[i] = find(electrode_counter $==$ electrodes_plotted); \%See if the electrode is active

if isempty(i) $==1 \%$ electrode of electrode_counter is not active, so plot $\%$ Get the name of the electrodes, match the name of the electrodes to the electrode position on the image electrode_position = find(strcmp(electrode_name(electrode_counter), electrode_matrix $(:, 2))==1)$;

$\%$ Plots the electrode in black $\operatorname{plot}($ electrodeXY(electrode_position,1), electrodeXY(electrode_position,2), 'o' , 'MarkerFaceColor', 'k', 'MarkerEdgeColor', 'k')

else \% electrode of electrode_counter is already plotted $\%$ Do nothing

157 end end

axis off $\operatorname{colormap}($ jet $(255))$ colorbar('YTick', [50:50:250], 'YTickLabel', \{num2str(ceil(50*10/255*(cmax-cmin)) $/ 10+\operatorname{ceil}(\mathrm{cmin} * 10) / 10), \operatorname{num} 2 \operatorname{str}(\operatorname{ceil}(100 * 10 / 255 *(\mathrm{cmax}-\mathrm{cmin})) / 10+\operatorname{ceil}(\mathrm{cmin}$ $\star 10) / 10), \operatorname{num} 2 \operatorname{str}(\operatorname{ceil}(150 * 10 / 255 *(\operatorname{cmax}-\operatorname{cmin})) / 10+\operatorname{ceil}(\operatorname{cmin} * 10) / 10)$, 
$\operatorname{num} 2 \operatorname{str}(\operatorname{ceil}(200 * 10 / 255 *(\operatorname{cmax}-\operatorname{cmin})) / 10+\operatorname{ceil}(\operatorname{cmin} * 10) / 10), \operatorname{strcat}('>$ ', $\operatorname{num} 2 \operatorname{str}(\operatorname{ceil}(250 * 10 / 255 *(\operatorname{cmax}-\operatorname{cmin})) / 10+\operatorname{ceil}(\operatorname{cmin} * 10) / 10))\})$;

file_type $=3$;

end

\section{A.2.2 make_movie}

\% Make Movie of Still Images

$$
\%
$$

4

function make_movie(movie_start_time,movie_stop_time,image_mode,excel_filename, analyzed_filename)

$\% \%$ Load the data for the movie

$\%$ Excel file with corresponding electrode names to map

$9[$ rx ,electrode_matrix $]=x \mathbf{l s r e a d}($ excel_filename,'Electrodes', 'A2:C93');

$\%$ Data and column electrode names

$\mathrm{R}=\operatorname{load}($ analyzed_filename,'electrode_name','SI_matrix', 's seizure_start_time ') ;

time_matrix = R.SI_matrix (movie_start_time:movie_stop_time,:,:);

electrode _name = R.electrode_name;

14 seizure_start_time $=$ R. seizure_start_time ;

$\% \%$ Put the data into the image at each time step

$\%$ This data is constant for each patient, throughout the movie

[rows,columns,pages] = size(time_matrix);

19 time_data $=$ reshape $($ time_matrix, 1 ,rows $*$ columns $*$ pages $)$;

time_data = sort $($ time_data $)$; 
time_data $(1$ :rows $*$ columns $)=[]$;

cmax = quantile(time_data,0.95);

${ }_{24} \% \mathrm{NaN}$ substitution where electrode is paired with itself

for $\mathrm{i}=1$ :length(electrode_name)

time_matrix $(:, \mathrm{i}, \mathrm{i})=\mathrm{NaN}$;

end

$\operatorname{cmin}=0$;

$29 \%$ Values for print out on bottom of movie/image

$\max \_$value $=\operatorname{nanmax}(\operatorname{nanmax}(\operatorname{nanmax}($ time_matrix $)))$;

$\operatorname{avg}$ _value $=$ nanmean $($ nanmean $($ nanmean $($ time_matrix $)))$;

$\min \_v a l u e=\operatorname{nanmin}(\operatorname{nanmin}(\operatorname{nanmin}($ time_matrix $)))$;

[rows,rx,ry] = size(time_matrix); \% Get the number of time points for movie

34

$\%$ Initialize the movie variable

$\mathrm{F}=[]$

number_of_frames $=12 ; \%$ Changes the length of the movie $\left(20^{\sim} 1 \mathrm{sec}\right)$

39 for time_point $=1$ :rows

[ file_type ] = make_image $($ electrode_matrix,electrode_name,squeeze(time_matrix( time_point,:,:)),cmin,cmax,image_mode);

$\operatorname{text}(500,50,[$ num 2str(movie_start_time-1+time_point),' (',num2str(movie_start_time -1+time_point-seizure_start_time),')'],'HorizontalAlignment','center')

$\operatorname{text}(200,675,[$ 'Min = ', num2str(min_value,'\%6.2f'),' \muV'],'HorizontalAlignment', ' center')

$\operatorname{text}\left(478,675,\left[{ }^{\prime} A v g={ }^{\prime}, \operatorname{num} 2 \operatorname{str}(\right.\right.$ avg_value, '\%6.2f'),' $\backslash$ muV'],'HorizontalAlignment',' center') 
$\operatorname{text}(776,675,[$ 'Max = ',num2str(max_value,'\%6.2f'),' \muV'],'HorizontalAlignment', ' center')

$\% \%$ After each image, capture the frame multiple times

$\mathrm{H}=\operatorname{getframe}($ gcf $)$;

for frame_counter $=1$ :number_of_frames

$$
\mathrm{F}=[\mathrm{F}, \mathrm{H}]
$$

$49 \quad$ end

close

end

$\% \%$ Save the full movie as an mpg

$\%$ Create a filename for the analyzed data

54 end_name = strfind(analyzed_filename,'.mat');

switch file_type

case 1

filename1 = strcat(analyzed_filename(1:end_name-1),' movie ${ }_{-}^{\prime}$, num 2 str( movie_start_time),' ', num 2str(movie_stop_time),'_all.mpg');

case 2

filename1 = strcat(analyzed_filename(1:end_name-1),' movie ${ }_{-}$, num 2 str( movie_start_time),' ', num2str(movie_stop_time),, ', num 2str(image_mode),'. mpg');

otherwise

filename1 = strcat(analyzed_filename(1:end_name-1),' movie ${ }_{-}^{\prime}$, num 2 str( movie_start_time),' ', num $2 \operatorname{str}\left(\right.$ movie_stop_time),' ', image_mode $\{1\},{ }_{-}{ }_{-}$, num2str(image_mode\{2\}),'.mpg');

end

mpgwrite(F, colormap,filename1);

64 close 


\section{A.2.3 still_image_2}

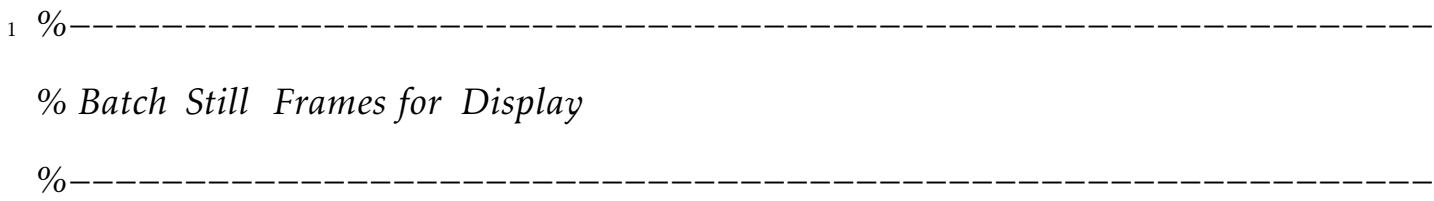

function still_image_2(movie_start_time,movie_stop_time,time_point,image_mode, excel_filename,analyzed_filename,sz_type,sz,sz_num,sz_start)

6

$\% \%$ Load the data for the movie

$\%$ Excel file with corresponding electrode names to map

[rx,electrode_matrix $]=\mathbf{x l s r e a d}($ excel_filename,'Electrodes', ' A2:C93');

$\%$ Data and column electrode names

$11 \mathrm{R}=$ load(analyzed_filename,'electrode_name','SI_matrix',' seizure_start_time' ) ;

time_matrix = R.SI_matrix (movie_start_time:movie_stop_time,:,:);

electrode_name = R.electrode_name;

$\% \%$ Put the data into the image at each time step

${ }_{16} \%$ This data is constant for each patient, throughout the movie

[rows, columns,pages] = size $($ time_matrix $)$;

time_data $=$ reshape $($ time_matrix, 1, rows $*$ columns $*$ pages $)$;

time_data $=$ sort $($ time_data $)$;

time_data $(1$ :rows $*$ columns $)=[]$;

21

cmax = quantile(time_data,0.95);

$\% N a N$ substitution, where electrode is paired to itself

for $\mathrm{i}=1$ :length(electrode_name)

time_matrix $(:, \mathrm{i}, \mathrm{i})=\mathrm{NaN}$; 


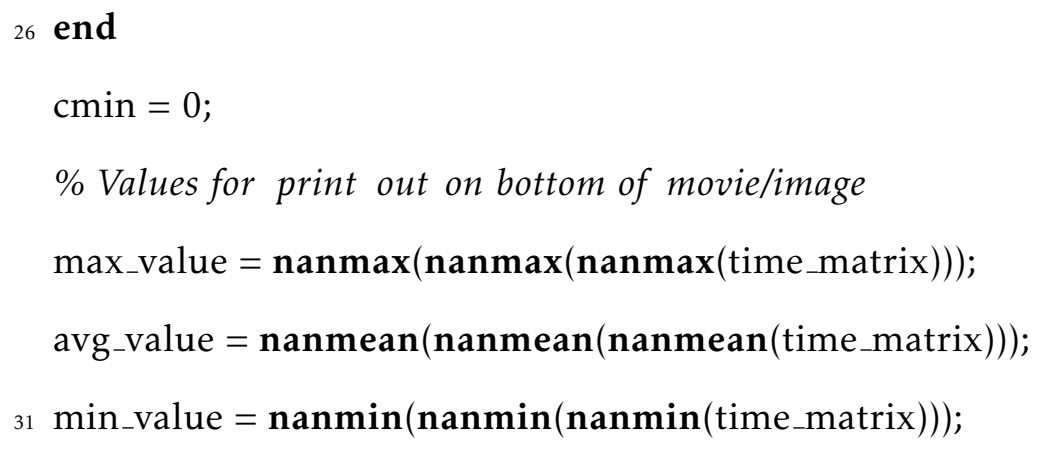

$\%$ Create a filename for the analyzed data

end_name = strfind (analyzed_filename,'.mat');

${ }_{41}$ switch file_type

case 1

filename1 = strcat(analyzed_filename(1:end_name-1),' ', num2str(time_point),' all.pdf');

case 2

filename1 = strcat $($ analyzed_filename(1:end_name-1),' ', num 2str(time_point),' ', , num2str(image_mode),'.pdf');

$46 \quad$ otherwise 


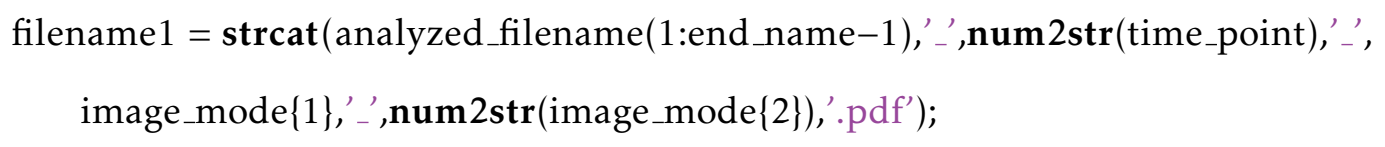

end

$\operatorname{print}\left({ }^{\prime}-\mathrm{dpdf}\right.$, filename1)

\section{A.2.4 Examples for the Different Image Modes}

Figure A.1 (following page): Examples of the three different display options for the make_image program. Two different time points during the seizure are represented for a seizure in Subject A. The three options are 1) to plot the highest SI value for each electrode connected to its pair (top row), 2) plot the highest SI pairs (in this case the top 70 SI pairs are plotted, middle row), and 3) plot the top SI connections for one designated electrode (in this case the top 20 SI connections for LH01, bottom row). Electrode that were present in the patients electrode grid, but were not in the highest SI values for modes 2 and 3 (middle and bottom row) were plotted in black. Plotting the highest SI pairs regardless of the which electrodes are involved can demonstrate localization of synchronization. In the rightmost column, the first mode displays disperse, unorganized connections (top, right), while the same time point in the second mode (middle, right) displays a concentration of high SI connections in the left temporal lobe. The third mode can be used to demonstrate changes in SI for an electrode. In the left column, the third mode shows connections from LH01 to LFA and RPT electrodes (left, bottom). At a different time point, the LH01 has only one connection to electrodes on the right side of the brain and most connections are in the left temporal lobe (right, bottom). 
392 (40)

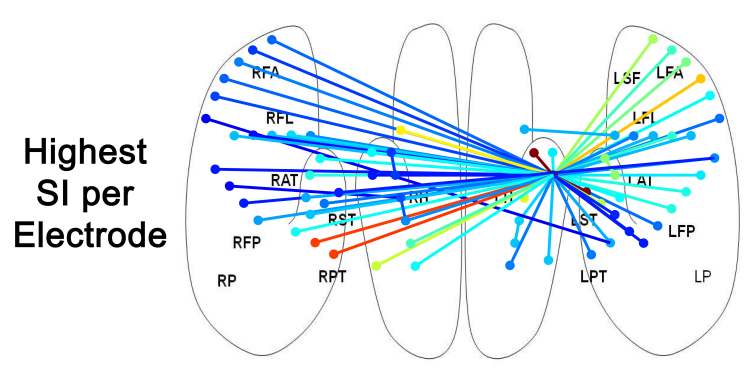

$\operatorname{Min}=0.73 \mu \mathrm{V} \quad$ Avg $=26.81 \mu \mathrm{V}$

$\operatorname{Max}=306.93 \mu \mathrm{V}$

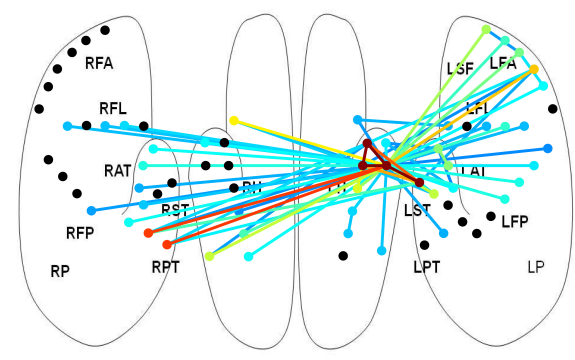

$\operatorname{Min}=0.73 \mu \vee$

$\operatorname{Avg}=26.81 \mu \mathrm{V}$

$\operatorname{Max}=306.93 \mu \mathrm{V}$

Highest

$70 \mathrm{SI}$

Values

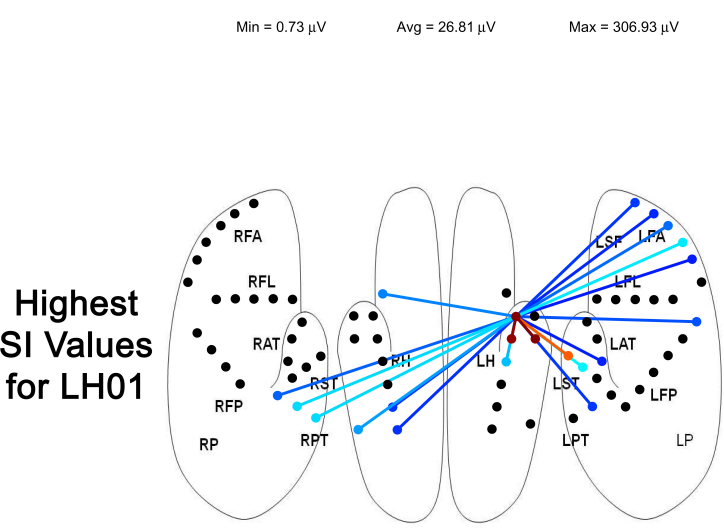

$\operatorname{Min}=0.73 \mu \mathrm{V}$

Avg $=26.81 \mu \mathrm{V}$

$\operatorname{Max}=306.93 \mu \mathrm{V}$
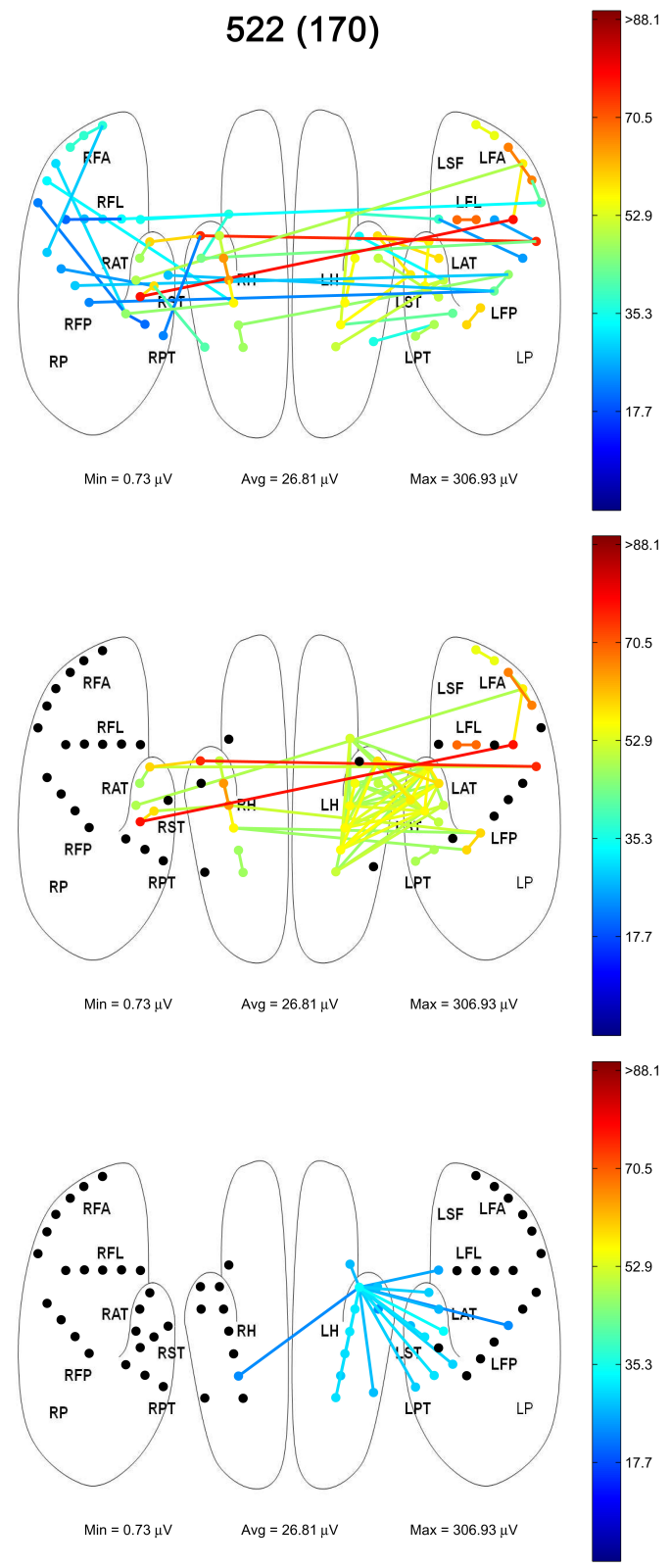


\section{Bibliography}

[1] Eric R. Kandel, James H. Schwartz, and Thomas M. Jessell. Principles of Neural Science. McGraw-Hill, New York, N.Y., 4th edition, 2000.

[2] Jerrold S. Meyer and Linda F. Quenzer. Psychopharmacology: Drugs, the Brain, and Behavior. Sinauer Associates, Inc., Sunderland, MA, 2005.

[3] T. R. Browne and G. L. Holmes. Handbook of Epilepsy. Lippincott, Williams \& Wilkins, Philidelphia, P.A., second edition, 2000.

[4] Andreas Schulze-Bonhage. Deep brain stimulation: A new approach to the treatment of epilepsy. Deutsches Ärzteblatt International, 106(24):407-412, 2009.

[5] E. H. Bertram, D. X. Zhang, P. Mangan, N. Fountain, and D. Rempe. Functional anatomy of limbic epilepsy: a proposal for central synchronization of a diffusely hyperexcitable network. Epilepsy Research, 32(1-2):194-205, 1998.

[6] Richard Wennberg, Francisco Arruda, Luis Felipe Quesney, and Andre Olivier. Preeminence of extrahippocampal structures in the generation of mesial temporal seizures: Evidence from human depth electrode recordings. Epilepsia, 43(7):716-726, 2002.

[7] Hal Blumenfeld, Maritza Rivera, J. Gabriel Vasquez, Akash Shah, Dina Ismail, 
Miro Enev, and Hitten P. Zaveri. Neocortical and thalamic spread of amygdala kindled seizures. Epilepsia, 48(2):254-262, 2007.

[8] Mojgan Hodaie, Richard A. Wennberg, Jonathan O. Dostrovsky, and Andres M. Lozano. Chronic anterior thalamus stimulation for intractable epilepsy. Epilepsia, 43(6):603-608, 2002.

[9] T. Wyckhuys, T. De Smedt, P. Claeys, R. Raedt, L. Waterschoot, K. Vonck, C. Van Den Broecke, C. Mabilde, L. Leybaert, W. Wadman, and P. Boon. High frequency deep brain stimulation in the hippocampus modifies seizure characteristics in kindled rats. Epilepsia, 48(8):1543-1550, 2007.

[10] R. Fisher, V. Salanova, T. Witt, R. Worth, T. Henry, R. Gross, K. Oommen, I. Osorio, J. Nazzaro, D. Labar, M. Kaplitt, M. Sperling, E. Sandok, J. Neal, A. Handforth, J. Stern, A. DeSalles, S. Chung, A. Shetter, D. Bergen, R. Bakay, J. Henderson, J. French, G. Baltuch, W. Rosenfeld, A. Youkilis, W. Marks, P. Garcia, N. Barbaro, N. Fountain, C. Bazil, R. Goodman, G. McKhann, K. B. Krishnamurthy, S. Papavassiliou, C. Epstein, J. Pollard, L. Tonder, J. Grebin, R. Coffey, and N. Graves. Electrical stimulation of the anterior nucleus of thalamus for treatment of refractory epilepsy. Epilepsia, 51(5):899-908, 2010.

[11] Robert J. Coffey. Deep brain stimulation devices: A brief technical history and review. Artificial Organs, 33(3):208-220, 2009.

[12] K. H. Lee, C. D. Blaha, P. A. Garris, P. Mohseni, A. E. Horne, K. E. Bennet, F. Agnesi, J. M. Bledsoe, D. B. Lester, C. Kimble, H. K. Min, Y. B. Kim, and Z. H. Cho. Evolution of deep brain stimulation: Human electrometer and smart devices supporting the next generation of therapy. Neuromodulation, 12(2):85-103, 2009. 
[13] J. Lockman and R. S. Fisher. Therapeutic brain stimulation for epilepsy. Neurologic Clinics, 27(4):1031-1040, 2009.

[14] C. Hauptmann, O. Popovych, and P. A. Tass. Effectively desynchronizing deep brain stimulation based on a coordinated delayed feedback stimulation via several sites: a computational study. Biological Cybernetics, 93(6):463-470, 2005.

[15] I. Z. Kiss, M. Quigg, S. H. C. Chun, H. Kori, and J. L. Hudson. Characterization of synchronization in interacting groups of oscillators: Application to seizures. Biophysical Journal, 94(3):1121-1130, 2008.

[16] John G. Nicholls, A. Robert Martin, Bruce G. Wallace, and Paul A. Fuchs. From Neuron to Brain. Sinauer Associates, Inc., Sunderland, MA, 4th edition, 2001.

[17] Dakota Rose Marshall, Laurie Filsrand, Carima Meintjies, and Lynelle Hagedorn. http://lynlaukimdak.wikispaces.com/09.+Nervous+System, accessed October 312011.

[18] Bernard Katz. Nerve, Muscle, and Synapse. McGraw-Hill, Inc, New York, NY, 1966.

[19] M. Barbarosie and M. Avoli. Ca3-driven hippocampal-entorhinal loop controls rather than sustains in vitro limbic seizures. Journal of Neuroscience, 17 (23):9308-9314, 1997.

[20] P. De Guzman, M. D’Antuono, and M. Avoli. Initiation of electrographic seizures by neuronal networks in entorhinal and perirhinal cortices in vitro. Neuroscience, 123(4):875-886, 2004. 
[21] P. A. Rutecki, R. G. Grossman, D. Armstrong, and S. Irishloewen. Electrophysiological connections between the hippocampus and entorhinal cortex in patients with complex partial seizures. Journal of Neurosurgery, 70(5): 667-675, 1989.

[22] The Brain Bank. http://thebrainbank.wordpress.com/2011/04/15/ seahorse-and-friends-a-stroll-down-memory-lane/, accessed October 312011.

[23] Isabel A. Muzzio, Clifford Kentros, and Eric Kandel. What is remembered? role of attention on the encoding and retrieval of hippocampal representations. The Journal of Physiology, 587(12):2837-2854, 2009.

[24] G. F. Ayala, Matsumot.H, and R. J. Gumnit. Excitability changes and inhibitory mechanisms in neocortical neurons during seizures. Journal of Neurophysiology, 33(1):73, 1970.

[25] London Health Science Center. http://www.lhsc.on.ca/eeg/epilepsy. htm, accessed October 312011.

[26] P. A. Tass. A model of desynchronizing deep brain stimulation with a demandcontrolled coordinated reset of neural subpopulations. Biological Cybernetics, 89(2):81-88, 2003.

[27] A. Gulyás-Kovács, J. Dóczi, I. Tarnawa, L. Détári, I. Banczerowski-Pelyhe, and I. Világi. Comparison of spontaneous and evoked epileptiform activity in three in vitro epilepsy models. Brain Research, 945(2):174-180, 2002.

[28] J. Ziburkus, J. R. Cressman, E. Barreto, and S. J. Schiff. Interneuron and pyramidal cell interplay during in vitro seizure-like events. Journal of Neurophysiology, 95(6):3948-3954, 2006. 
[29] R. D. Traub, A. Bibbig, F. E. N. LeBeau, E. H. Buhl, and M. A. Whittington. Cellular mechanisms of neuronal population oscillations in the hippocampus in vitro. Annual Review of Neuroscience, 27:247-278, 2004.

[30] T. I. Netoff and S. J. Schiff. Decreased neuronal synchronization during experimental seizures. Journal of Neuroscience, 22(16):7297-7307, 2002.

[31] H. L. Haas and J. G. R. Jefferys. Low-calcium field burst discharges of ca1 pyramidal neurons in rat hippocampal slices. Journal of Physiology-London, 354(SEP):185-201, 1984.

[32] R. Bianchi and R. K. S. Wong. Carbachol-induced synchronized rhythmic bursts in ca3 neurons of guinea-pig hippocampus in-vitro. Journal of Neurophysiology, 72(1):131-138, 1994.

[33] I. Niespodziany, H. Klitgaard, and D. G. Margineanu. Desynchronizing effect of levetiracetam on epileptiform responses in rat hippocampal slices. Neuroreport, 14(9):1273-1276, 2003.

[34] R. D. Traub, J. G. R. Jefferys, and M. A. Whittington. Enhanced nmda conductance can account for epileptiform activity-induced by low $\mathrm{mg} 2+$ in the rat hippocampal slice. Journal of Physiology-London, 478(3):379-393, 1994.

[35] R. Miles and R. K. S. Wong. Single neurons can initiate synchronized population discharge in the hippocampus. Nature, 306(5941):371-373, 1983.

[36] R. Miles, R. K. S. Wong, and R. D. Traub. Synchronized afterdischarges in the hippocampus - contribution of local synaptic-interactions. Neuroscience, 12 (4):1179-1189, 1984. 
[37] Y. Fujiwara-Tsukamoto, Y. Isomura, K. Kaneda, and M. Takada. Synaptic interactions between pyramidal cells and interneurone subtypes during seizure-like activity in the rat hippocampus. Journal of Physiology-London, 557(3):961-979, 2004.

[38] H. E. Scharfman. Synchronization of area ca3 hippocampal pyramidal cells and non-granule cells of the dentate gyrus in bicuculline-treated rat hippocampal slices. Neuroscience, 59(2):245-257, 1994.

[39] A. S. Pikovsky, M. G. Rosenblum, and J. Kurths. Synchronization: A Universal Concept in Nonlinear Science. Cambridge University Press, Cambridge, 2001.

[40] Sarah Johnson. Characterization of synchronization patterns in induced seizures. Master's thesis, University of Virginia, 2008.

[41] Nicholas M. Barbaro, Mark Quigg, Donna K. Broshek, Mariann M. Ward, Kathleen R. Latnborn, Kenneth D. Laxer, David A. Larson, William Dillon, Lynn Verhey, Paul Garcia, Ladislau Steiner, Christine Heck, Douglas Kondziolka, Robert Beach, William Olivero, Thomas C. Witt, Vicenta Salanova, and Robert Goodman. A multicenter, prospective pilot study of gamma knife radiosurgery for mesial temporal lobe epilepsy: Seizure response, adverse events, and verbal memory. Annals of Neurology, 65(2):167-175, 2009.

[42] S. S. Spencer, A. T. Berg, B. G. Vickrey, M. R. Sperling, C. W. Bazil, S. Shinnar, J. T. Langfitt, T. S. Walczak, and S. V. Pacia. Predicting long-term seizure outcome after resective epilepsy surgery - the multicenter study. Neurology, 65(6):912-918, 2005.

[43] S. Wiebe, W. T. Blume, J. P. Girvin, and M. Eliasziw. A randomized, controlled 
trial of surgery for temporal-lobe epilepsy. New England Journal of Medicine, 345(5):311-318, 2001.

[44] S. S. Spencer. Neural networks in human epilepsy: Evidence of and implications for treatment. Epilepsia, 43(3):219-227, 2002.

[45] A. Bragin, C. L. Wilson, and J. Engel. Chronic epileptogenesis requires development of a network of pathologically interconnected neuron clusters: A hypothesis. Epilepsia, 41:S144-S152, 2000.

[46] J. Bear, N. B. Fountain, and E. W. Lothman. Responses of the superficial entorhinal cortex in vitro in slices from naive and chronically epileptic rats. Journal of Neurophysiology, 76(5):2928-2940, 1996.

[47] Gaelle Bettus, Eric Guedj, Florian Joyeux, Sylviane Confort-Gouny, Elisabeth Soulier, Virginie Laguitton, Patrick J. Cozzone, Patrick Chauvel, Jean-Philippe Ranjeva, Fabrice Bartolomei, and Maxime Guye. Decreased basal fmri functional connectivity in epileptogenic networks and contralateral compensatory mechanisms. Human Brain Mapping, 30(5):1580-1591, 2009.

[48] E. H. Bertram. Why does surgery fail to cure limbic epilepsy? seizure functional anatomy may hold the answer. Epilepsy Research, 56(2-3):93-99, 2003.

[49] A. Ossadtchi, R. E. Greenblatt, V. L. Towle, M. H. Kohrman, and K. Kamada. Inferring spatiotemporal network patterns from intracranial eeg data. Clinical Neurophysiology, 121(6):823-835, 2010.

[50] Luc Valton, Maxime Guye, Aileen McGonigal, Patrick Marquis, Fabrice Wendling, Jean Regis, Patrick Chauvel, and Fabrice Bartolomei. Functional 
interactions in brain networks underlying epileptic seizures in bilateral diffuse periventricular heterotopia. Clinical Neurophysiology, 119(1):212-223, 2008.

[51] L. D. Iasemidis, J. C. Sackellares, Hitten P. Zaveri, and W. J. Williams. Phase space topography and the lyapunov exponent of electrocorticograms in partial seizures. Brain Topography, 2:187-201, 1990.

[52] Fabrice Wendling, Patrick Chauvel, Arnaud Biraben, and Fabrice Bartolomei. From intracerebral eeg signals to brain connectivity: identification of epileptogenic networks in partial epilepsy. Frontiers in systems neuroscience, 4:154, 2010.

[53] Mark A. Kramer, Uri T. Eden, Eric D. Kolaczyk, Rodrigo Zepeda, Emad N. Eskandar, and Sydney S. Cash. Coalescence and fragmentation of cortical networks during focal seizures. Journal of Neuroscience, 30(30):10076-10085, 2010.

[54] Pieter van Mierlo, Evelien Carrette, Hans Hallez, Kristl Vonck, Dirk Van Roost, Paul Boon, and Steven Staelens. Accurate epileptogenic focus localization through time-variant functional connectivity analysis of intracranial electroencephalographic signals. Neuroimage, 56(3):1122-1133, 2011.

[55] Christopher P. Warren, Sanqing Hu, Matt Stead, Benjamin H. Brinkmann, Mark R. Bower, and Gregory A. Worrell. Synchrony in normal and focal epileptic brain: The seizure onset zone is functionally disconnected. Journal of Neurophysiology, 104(6):3530-3539, 2010.

[56] L. Ding, GA Worrell, TD Lagerlund, and B He. Spatio-temporal source 
localization and granger causality in ictal source analysis. In IEEE Eng Med Bio Soc, volume 1, pages 3670-3671, 2006.

[57] Fabrice Bartolomei, Delphine Cosandier-Rimele, Aileen McGonigal, Sandrine Aubert, Jean Regis, Martine Gavaret, Fabrice Wendling, and Patrick Chauvel. From mesial temporal lobe to temporoperisylvian seizures: A quantified study of temporal lobe seizure networks. Epilepsia, 51(10):2147-2158, 2010.

[58] Premysl Jiruska, Jozsef Csicsvari, Andrew D. Powell, John E. Fox, Wei-Chih Chang, Martin Vreugdenhil, Xiaoli Li, Milan Palus, Alejandro F. Bujan, Richard W. Dearden, and John G. R. Jefferys. High-frequency network activity, global increase in neuronal activity, and synchrony expansion precede epileptic seizures in vitro. Journal of Neuroscience, 30(16):5690-5701, 2010.

[59] Alex J. Cadotte, Thomas H. Mareci, Thomas B. DeMarse, Mansi B. Parekh, Rajasimhan Rajagovindan, William L. Ditto, Sachin S. Talathi, Dong-Uk Hwang, and Paul R. Carney. Temporal lobe epilepsy: Anatomical and effective connectivity. Ieee Transactions on Neural Systems and Rehabilitation Engineering, 17(3):214-223, 2009.

[60] Gaelle Bettus, Fabrice Wendling, Maxime Guye, Luc Valton, Jean Regis, Patrick Chauvel, and Fabrice Bartolomei. Enhanced eeg functional connectivity in mesial temporal lobe epilepsy. Epilepsy Research, 81(1):58-68, 2008.

[61] G. U. Martz, S. E. Johnson, J. L. Hudson, and M. Quigg. Utility of synchrony index for localization of seizure onset, 2008. AES Annual Meeting, Abstract 1.046.

[62] P. Tass, M. G. Rosenblum, J. Weule, J. Kurths, A. Pikovsky, J. Volkmann, 
A. Schnitzler, and H.-J. Freund. Detection of n:m phase locking from noisy data: Application to magnetoencephalography. Physical Review Letters, 81 (15):3291-3294, 1998.

[63] C. Y. Kramer. Extension of multiple range tests to group means with unequal number of replications. Biometrics, 12:307-310, 1956.

[64] Dong W. Kim, Sang K. Lee, Hyunwoo Nam, Kon Chu, Chun K. Chung, SeoYoung Lee, Geeyoung Choe, and Hyun K. Kim. Epilepsy with dual pathology: Surgical treatment of cortical dysplasia accompanied by hippocampal sclerosis. Epilepsia, 51(8):1429-1435, 2010.

[65] B. Diehl, I. Najm, A. Mohamed, T. Babb, Z. Ying, and W. Bingaman. Interictal eeg, hippocampal atrophy, and cell densities in hippocampal sclerosis and hippocampal sclerosis associated with microscopic cortical dysplasia. Journal of Clinical Neurophysiology, 19(2):157-162, 2002.

[66] C. Wilke, G. A. Worrell, and B. He. Analysis of epileptogenic network properties during ictal activity. In IEEE Eng Med Biol Soc, pages 2220-2223, 2009.

[67] Maryann D’Alessandro, George Vachtsevanos, Rosana Esteller, Javier Echauz, Stephen Cranstoun, Greg Worrell, Landi Parish, and Brian Litt. A multifeature and multi-channel univariate selection process for seizure prediction. Clinical Neurophysiology, 116(3):506-516, 2005.

[68] S. S. Spencer, D. Marks, A. Katz, J. Kim, and D. D. Spencer. Anatomic correlates of interhippocampal seizure propagation time. Epilepsia, 33(5): 862-873, 1992. 
[69] N. F. Moran, L. Lemieux, N. D. Kitchen, D. R. Fish, and S. D. Shorvon. Extrahippocampal temporal lobe atrophy in temporal lobe epilepsy and mesial temporal sclerosis. Brain, 124:167-175, 2001.

[70] Leonardo Bonilha, Andrea Alessio, Chris Rorden, Gordon Baylis, Benito P. Damasceno, Li Li Min, and Fernando Cendes. Extrahippocampal gray matter atrophy and memory impairment in patients with medial temporal lobe epilepsy. Human Brain Mapping, 28(12):1376-1390, 2007.

[71] M. Avoli. Do interictal discharges promote or control seizures? experimental evidence from an in vitro model of epileptiform discharge. Epilepsia, 42:2-4, 2001.

[72] David Sullivan, Jozsef Csicsvari, Kenji Mizuseki, Sean Montgomery, Kamran Diba, and Gyoergy Buzsaki. Relationships between hippocampal sharp waves, ripples, and fast gamma oscillation: Influence of dentate and entorhinal cortical activity. Journal of Neuroscience, 31(23):8605-8616, 2011.

[73] N. B. Fountain, J. Bear, E. H. Bertram, and E. W. Lothman. Responses of deep entorhinal cortex are epileptiform in an electrogenic rat model of chronic temporal lobe epilepsy. Journal of Neurophysiology, 80(1):230-240, 1998.

[74] Alfons Schnitzler and Joachim Gross. Normal and pathological oscillatory communication in the brain. Nat Rev Neurosci, 6(4):285-296, 2005.

[75] P. J. Uhlhaas and W. Singer. Neural synchrony in brain disorders: Relevance for cognitive dysfunctions and pathophysiology. Neuron, 52(1):155-168, 2006.

[76] J. Artieda, M. Alegre, M. Valencia, E. Urrestarazu, M. Perez-Alcazar, M. J. Nicolas, J. L. Azcarate, and J. Iriarte. Brain oscillations: pathophysiological 
and therapeutic role in some neurological and psychiatric diseases. Anales Del Sistema Sanitario De Navarra, 32:45-60, 2009.

[77] A. Pogosyan, F. Yoshida, C. C. Chen, I. Martinez-Torres, T. Foltynie, P. Limousin, L. Zrinzo, M. I. Hariz, and P. Brown. Parkinsonian impairment correlates with spatially extensive subthalamic oscillatory synchronization. Neuroscience, 171(1):245-257, 2010.

[78] G. C. Albert, C. M. Cook, F. S. Prato, and A. W. Thomas. Deep brain stimulation, vagal nerve stimulation and transcranial stimulation: An overview of stimulation parameters and neurotransmitter release. Neuroscience and Biobehavioral Reviews, 33(7):1042-1060, 2009.

[79] D. A. Wagenaar, R. Madhavan, J. Pine, and S. M. Potter. Controlling bursting in cortical cultures with closed-loop multi-electrode stimulation. Journal of Neuroscience, 25(3):680-688, 2005.

[80] B. J. Gluckman, H. Nguyen, S. L. Weinstein, and S. J. Schiff. Adaptive electric field control of epileptic seizures. Journal of Neuroscience, 21(2):590-600, 2001.

[81] P. J. Franaszczuk, P. Kudela, and G. K. Bergey. External excitatory stimuli can terminate bursting in neural network models. Epilepsy Research, 53(1-2): 65-80, 2003.

[82] E. Scholl, G. Hiller, P. Hovel, and M. A. Dahlem. Time-delayed feedback in neurosystems. Philosophical Transactions of the Royal Society a-Mathematical Physical and Engineering Sciences, 367(1891):1079-1096, 2009.

[83] C. A. Batista, S. R. Lopes, R. L. Viana, and A. M. Batista. Delayed feedback 
control of bursting synchronization in a scale-free neuronal network. Neural Netw, 23(1):114-24, 2010.

[84] C. Hauptmann, O. Popovych, and P. A. Tass. Delayed feedback control of synchronization in locally coupled neuronal networks. Neurocomputing, 65-66:759-767, 2005.

[85] O. V. Popovych, C. Hauptmann, and P. A. Tass. Effective desynchronization by nonlinear delayed feedback. Phys. Rev. Lett., 94:164102, 2005.

[86] O. V. Popovych, C. Hauptmann, and P. A. Tass. Control of neuronal synchrony by nonlinear delayed feedback. Biol. Cybern., 95:69, 2006.

[87] S. Santaniello, G. Fiengo, L. Glielmo, and W. M. Grill. Closed-loop control of deep brain stimulation: A simulation study. Ieee Transactions on Neural Systems and Rehabilitation Engineering, 19(1):15-24, 2011.

[88] R. J. Andrews. Neuromodulation advances in the next five years. Annals of the New York Academy of Sciences, 1199:204-211, 2010.

[89] T. I. Netoff, M. I. Banks, A. D. Dorval, C. D. Acker, J. S. Haas, N. Kopell, and J. A. White. Synchronization in hybrid neuronal networks of the hippocampal formation. Journal of Neurophysiology, 93(3):1197-1208, 2005.

[90] I. Z. Kiss, C. G. Rusin, H. Kori, and J. L. Hudson. Engineering complex dynamical structures: Sequential patterns and desynchronization. Science, 316(5833):1886-1889, 2007.

[91] C. G. Rusin, I. Z. Kiss, H. Kori, and J. L. Hudson. Framework for engineering the collective behavior of complex rhythmic systems. Industrial \& Engineering Chemistry Research, 48(21):9416-9422, 2009. 
[92] R. F. Galán, G. B. Ermentrout, and N. N. Urban. Efficient estimation of phase-resetting curves in real neurons and its significance for neural-network modeling. Physical Review Letters, 94(15), 2005.

[93] J. A. Goldberg, C. A. Deister, and C. J. Wilson. Response properties and synchronization of rhythmically firing dendritic neurons. Journal of Neurophysiology, 97(1):208-219, 2007.

[94] F. H. Sieling, C. C. Canavier, and A. A. Prinz. Predictions of phase-locking in excitatory hybrid networks: Excitation does not promote phase-locking in pattern-generating networks as reliably as inhibition. Journal of Neurophysiology, 102(1):69-84, 2009.

[95] I. Tateno and H. P. C. Robinson. Quantifying noise-induced stability of a cortical fast-spiking cell model with kv3-channell-like current. Biosystems, 89(1-3):110-116, 2007.

[96] Susanna C. Manirubia, Alexander S. Mikhailov, and Damian H. Zanette. Emergence of dynamical order: synchronization phenomena in complex systems, volume 2 of World Scientific lecture notes in complex systems. World Scientific, River Edge, N.J., 2004.

[97] C. D. Acker, N. Kopell, and J. A. White. Synchronization of strongly coupled excitatory neurons: Relating network behavior to biophysics. Journal of Computational Neuroscience, 15(1):71-90, 2003.

[98] B. S. Gutkin, G. B. Ermentrout, and A. D. Reyes. Phase-response curves give the responses of neurons to transient inputs. Journal of Neurophysiology, 94 (2):1623-1635, 2005. 
[99] T. I. Netoff, C. D. Acker, J. C. Bettencourt, and J. A. White. Beyond two-cell networks: Experimental measurement of neuronal responses to multiple synaptic inputs. Journal of Computational Neuroscience, 18(3):287-295, 2005.

[100] Amanda J. Preyer and Robert J. Butera. Neuronal oscillators in aplysia californica that demonstrate weak coupling in vitro. Physical Review Letters, 95:138103, 2005.

[101] T. Tateno and H. P. C. Robinson. Phase resetting curves and oscillatory stability in interneurons of rat somatosensory cortex. Biophysical Journal, 92 (2):683-695, 2007.

[102] Y. Tsubo, M. Takada, A. D. Reyes, and T. Fukai. Layer and frequency dependencies of phase response properties of pyramidal neurons in rat motor cortex. European Journal of Neuroscience, 25(11):3429-3441, 2007.

[103] Y. Kawamura, H. Nakao, K. Arai, H. Kori, and Y. Kuramoto. Collective phase sensitivity. Physical Review Letters, 101(2):024101, 2008.

[104] N. Nakagawa and Y. Kuramoto. Collective chaos in a population of globally coupled oscillators. Progress of Theoretical Physics, 89(2):313-323, 1993.

[105] J. X. Cui, C. C. Canavier, and R. J. Butera. Functional phase response curves: A method for understanding synchronization of adapting neurons. Journal of Neurophysiology, 102(1):387-398, 2009.

[106] R. F. Galán, G. B. Ermentrout, and N. N. Urban. Predicting synchronized neural assemblies from experimentally estimated phase-resetting curves. Neurocomputing, 69(10-12):1112-1115, 2006. 
[107] E. M. Izhikevich. Dynamical Systems in Neuroscience: The Geometry of Excitability and Bursting. MIT Press, Cambridge, Mass., 2007.

[108] K. Ota, M. Nomura, and T. Aoyagi. Weighted spike-triggered average of a fluctuating stimulus yielding the phase response curve. Physical Review Letters, 103(2), 2009.

[109] Arthur T. Winfree. The Geometry of Biological Time. Springer, New York, NY, 1980.

[110] Yoshiki Kuramoto. Chemical Oscillations, Waves and Turbulence. Springer, New York, NY, 1984.

[111] I. Z. Kiss, Y. Zhai, and J. L. Hudson. Predicting mutual entrainment of oscillators with experiment-based phase models. Phys. Rev. Lett., 94:248301, 2005.

[112] J. Miyazaki and S. Kinoshita. Determination of a coupling function in multicoupled oscillators. Physical Review Letters, 96(19), 2006.

[113] C. G. Rusin, I. Tokuda, I. Z. Kiss, and J. L. Hudson. Engineering of synchronization and clustering of a population of chaotic chemical oscillators. Angewandte Chemie, 50:10212, 2011.

[114] J. Miyazaki and S. Kinoshita. Method for determining a coupling function in coupled oscillators with application to belousov-zhabotinsky oscillators. Physical Review E, 74(5), 2006.

[115] Craig G. Rusin. Engineering the Behavior of Complex Rhythmic Systems. PhD thesis, University of Virginia, 2009. 
[116] Gary Banker and Kimberly Goslin, editors. Culturing Nerve Cells. MIT Press, Cambridge, Mass, 2nd edition, 1998.

[117] Joseph Nunez. Primary culture of hippocampal neurons from p0 newborn rats. Journal of Visualized Experiments, 19, 2008.

[118] Areles Molleman. Patch Clamping: An Introductory Guide to Patch Clamp Electrophysiology. John Weily \& Sons LTD, West Sussex, England, 2003.

[119] A. D. Dorval, D. J. Christini, and J. A. White. Real-time linux dynamic clamp: A fast and flexible way to construct virtual ion channels in living cells. Annals of Biomedical Engineering, 29(10):897-907, 2001.

[120] T. Nowotny, A. Szucs, R. D. Pinto, and A. I. Selverston. Stdpc: A modern dynamic clamp. Journal of Neuroscience Methods, 158(2):287-299, 2006.

[121] K. Okuda. Variety and generality of clustering in globally coupled oscillators. Physica D, 63:424, 1993.

[122] S. C. Manrubia, A. S. Mikhailov, and D. H. Zanette. Emergence of Dynamical Order: Synhronization Phenomena in Complex Systems. World Scientific Sigapore, 2004.

[123] T. Nowotny, V. P. Zhigulin, A. I. Selverston, H. D. I. Abarbanel, and M. I. Rabinovich. Enhancement of synchronization in a hybrid neural circuit by spike-timing dependent plasticity. Journal of Neuroscience, 23(30):9776-9785, 2003.

[124] N. Kopell and B. Ermentrout. Chemical and electrical synapses perform complementary roles in the synchronization of interneuronal networks. Pro- 
ceedings of the National Academy of Sciences of the United States of America, 101 (43):15482-15487, 2004.

[125] J. G. Mancilla, T. J. Lewis, D. J. Pinto, J. Rinzel, and B. W. Connors. Synchronization of electrically coupled pairs of inhibitory interneurons in neocortex. Journal of Neuroscience, 27(8):2058-2073, 2007.

[126] J. Suzurikawa, M. Nakao, Y. Jimbo, R. Kanzaki, and H. Takahashi. Lightaddressed stimulation under ca2+ imaging of cultured neurons. Ieee Transactions on Biomedical Engineering, 56(11):2660-2665, 2009.

[127] D. Hansel, G. Mato, and C. Meunier. Phase dynamics for weakly coupled hodgkin-huxley neurons. Europhysics Letters, 23(5):367-372, 1993.

[128] J. Wang, B. Deng, and X. Y. Fei. Chaotic synchronization of two coupled neurons via nonlinear control in external electrical stimulation. Chaos Solitons E Fractals, 27(5):1272-1278, 2006.

[129] J. L. P. Velazquez, R. F. Galan, L. G. Dominguez, Y. Leshchenko, S. Lo, J. Belkas, and R. G. Erra. Phase response curves in the characterization of epileptiform activity. Physical Review E, 76(6), 2007.

[130] D. Golomb, A. Shedmi, R. Curtu, and G. B. Ermentrout. Persistent synchronized bursting activity in cortical tissues with low magnesium concentration: A modeling study. Journal of Neurophysiology, 95(2):1049-1067, 2006.

[131] R. D. Traub, R. Miles, and R. K. S. Wong. Models of synchronized hippocampal bursts in the presence of inhibition .1. single population events. Journal of Neurophysiology, 58(4):739-751, 1987. 
[132] H. P. C. Robinson, M. Kawahara, Y. Jimbo, K. Torimitsu, Y. Kuroda, and A. Kawana. Periodic synchronized bursting and intracellular calcium transients elicited by low magnesium in cultured cortical-neurons. Journal of Neurophysiology, 70(4):1606-1616, 1993.

[133] C. Borgers and N. Kopell. Synchronization in networks of excitatory and inhibitory neurons with sparse, random connectivity. Neural Computation, 15 (3):509-538, 2003.

[134] Daniel Johnston and Samuel Mia-Sin Wu. Foundations of Cellular Neurophysiology. The MIT Press, Cambridge, MA, 1995.

[135] P. S. Mangan and J. Kapur. Factors underlying bursting behavior in a network of cultured hippocampal neurons exposed to zero magnesium. Journal of Neurophysiology, 91(2):946-957, 2004.

[136] R. Q. Quiroga, T. Kreuz, and P. Grassberger. Event synchronization: A simple and fast method to measure synchronicity and time delay patterns. Physical Review E, 66(4), 2002.

[137] M. Bykhovskaia. Making quantal analysis more convenient, fast, and accurate: User-friendly software quantan. Journal of Neuroscience Methods, 168(2):500$513,2008$.

[138] V. Pasquale, S. Martinoia, and M. Chiappalone. A self-adapting approach for the detection of bursts and network bursts in neuronal cultures. Journal of Computational Neuroscience, 29(1-2):213-229, 2010.

[139] J. V. Selinger, N. V. Kulagina, T. J. O’Shaughnessy, W. Ma, and J. J. Pancrazio. Methods for characterizing interspike intervals and identifying bursts in neuronal activity. Journal of Neuroscience Methods, 162(1-2):64-71, 2007. 
[140] J. H. Cocatre-Zilgien and F. Delcomyn. Identification of bursts in spike trains. Journal of Neuroscience Methods, 41(1):19-30, 1992.

[141] D. L. Benson, F. H. Watkins, O. Steward, and G. Banker. Characterization of gabaergic neurons in hippocampal cell-cultures. Journal of Neurocytology, 23 (5):279-295, 1994.

[142] David Lyttle and Jean-Marc Fellous. A new similarity measure for spike trains: Sensitivity to bursts and periods of inhibition. Journal of Neuroscience Methods, 199(2):296-309, 2011.

[143] R. D. Traub, W. D. Knowles, R. Miles, and R. K. S. Wong. Synchronized afterdischarges in the hippocampus - simulation studies of the cellular mechanism. Neuroscience, 12(4):1191-1200, 1984.

[144] Y. Yaari, M. E. Selzer, and J. H. Pincus. Phenytoin - mechanisms of its anticonvulsant action. Annals of Neurology, 20(2):171-184, 1986.

[145] R. L. Macdonald and K. M. Kelly. Antiepileptic drug mechanisms of action. Epilepsia, 34:S1-S8, 1993.

[146] S. D. Donevan and M. A. Rogawski. Gyki 52466, a 2,3-benzodiazepine, is a highly selective, noncompetitive antagonist of ampa/kainate receptor responses. Neuron, 10(1):51-59, 1993.

[147] M. Madeja, D. G. Margineanu, A. Gorji, E. Siep, P. Boerrigter, H. Klitgaard, and E. J. Speckmann. Reduction of voltage-operated potassium currents by levetiracetam: a novel antiepileptic mechanism of action? Neuropharmacology, 45(5):661-671, 2003. 
[148] C. Y. Lee, C. C. Chen, and H. H. Liou. Levetiracetam inhibits glutamate transmission through presynaptic p/q-type calcium channels on the granule cells of the dentate gyrus. British Journal of Pharmacology, 158(7):1753-1762, 2009.

[149] B. A. Lynch, N. Lambeng, K. Nocka, P. Kensel-Hammes, S. M. Bajjalieh, A. Matagne, and B. Fuks. The synaptic vesicle protein sv2a is the binding site for the antiepileptic drug levetiracetam. Proceedings of the National Academy of Sciences of the United States of America, 101(26):9861-9866, 2004.

[150] K. L. Custer, N. S. Austin, J. M. Sullivan, and S. M. Bajjalieh. Synaptic vesicle protein 2 enhances release probability at quiescent synapses. Journal of Neuroscience, 26(4):1303-1313, 2006.

[151] P. P. Quilichini, D. Diabira, C. Chiron, M. Milh, Y. Ben-Ari, and H. Gozlan. Effects of antiepileptic drugs on refractory seizures in the intact immature corticohippocampal formation in vitro. Epilepsia, 44(11):1365-1374, 2003.

[152] K. Albus, A. Wahab, and U. Heinemann. Standard antiepileptic drugs fail to block epileptiform activity in rat organotypic hippocampal slice cultures. British Journal of Pharmacology, 154(3):709-724, 2008.

[153] T. Kreuz, F. Mormann, R. G. Andrzejak, A. Kraskov, K. Lehnertz, and P. Grassberger. Measuring synchronization in coupled model systems: A comparison of different approaches. Physica D-Nonlinear Phenomena, 225(1):29-42, 2007.

[154] X. Shi and Q. S. Lu. Burst synchronization of electrically and chemically coupled map-based neurons. Physica A-Statistical Mechanics and Its Applications, 388(12):2410-2419, 2009. 
[155] N. Buric, K. Todorovic, and N. Vasovic. Exact synchronization of noisy bursting neurons with coupling delays. Chaos Solitons E Fractals, 40(3): 1127-1135, 2009.

[156] M. P. Kaplan, K. S. Wilcox, and M. A. Dichter. Differences in multiple forms of short-term plasticity between excitatory and inhibitory hippocampal neurons in culture. Synapse, 50(1):41-52, 2003.

[157] M. A. Kramer, E. D. Kolaczyk, and H. E. Kirsch. Emergent network topology at seizure onset in humans. Epilepsy Research, 79(2-3):173-186, 2008.

[158] A. Maximov, Z. P. P. Pang, D. G. R. Tervo, and T. C. Sudhof. Monitoring synaptic transmission in primary neuronal cultures using local extracellular stimulation. Journal of Neuroscience Methods, 161(1):75-87, 2007.

[159] Xin Ren. Dynamic analysis of hippocampal neuronal synchronization in a rat seizure model. Master's thesis, University of Virginia, 2011.

[160] M. E. Colpan, Y. Li, J. Dwyer, and D. J. Mogul. Proportional feedback stimulation for seizure control in rats. Epilepsia, 48(8):1594-1603, 2007. 


\section{List of Publications Based on the}

\section{Dissertation}

1. S.E. Johnson, J.L. Hudson and J. Kapur, "Synchronization of Action Potentials During Low Magnesium Bursting" J. Physiol., (in preparation).

2. C.G. Rusin, S.E. Johnson, J. Kapur and J.L. Hudson, "Engineering the Synchronization of Neuron Action Potentials Using Global Time-delayed Feedback Stimulation" Phys. Rev. E, 84, 066202, 2011.

3. G.U. Martz, S.E. Johnson, X. Liu, B.J. Wolf, J.L. Hudson and M. Quigg, "Display of Ictal Hippocampal-Temporal Cortical Network Dynamics in Refractory Mesial Temporal Lobe Epilepsy." Epilepsy Res., (submitted). 